\title{
Computer Simulations as Scientific Instruments
}

By

(C) 2020

Ramón Alvarado

Ph.D., University of Kansas, 2020

M.A., University of Kansas, 2017

M.A., University of Texas at El Paso, 2014

B.A., University of Texas at El Paso, 2011

Submitted to the graduate degree program in Philosophy and the Graduate Faculty of the University of Kansas in partial fulfillment of the requirements for the degree of Doctor of Philosophy.

Chair: John Symons

Armin Schulz

Corey Maley

Perry Alexander

Paul Humphreys

Date Defended: $14^{\text {th }}$, February, 2020 
The dissertation committee for Ramón Alvarado certifies that this is the approved version of the following dissertation:

\section{Computer Simulations as Scientific Instruments}

Chair: John Symons

Date Approved: February $14^{\text {th }}, 2020$ 


\begin{abstract}
Although now ubiquitous in scientific inquiry, the role and significance of the use of computer simulations in science is still widely debated. Questions that arise in the philosophical literature on computer simulations include how computer simulations relate to other elements of scientific inquiry, what they do in relation to other methods, practices and devices, and how we can justifiably trust them. Recent debates in the epistemology of computer simulations have attempted to answer these questions by starting with an assumed dichotomy that characterizes simulations either as extensions of formal methods - given the mathematical content they manipulate and yield — or as special forms of experiments (and their associated practices) capable of yielding empirical information about the systems they simulate. In this dissertation I contend that computer simulations are neither. I argue that these views of computer simulations fail to capture the independence and hence distinctiveness of computer simulations in terms of their functions and their non-identity with the elements of scientific inquiry they are conventionally subsumed under. In other words, I will argue that computer simulations are not the formal methods that they implement, they are not the experiments they can simulate, and they are not the practices associated with them either. Rather, I argue, they are the technical artifacts, of the kind we usually denominate as instruments, with which the above tasks and practices can be carried out. This is the 'instrument view' of computer simulations.
\end{abstract}

Philosophical discussions on the nature of computer simulations have successfully, and in my opinion correctly, moved away from a purely formal treatment of computer simulations. Computer simulations, in short, are now acknowledged to be more than the equations and theories they are based upon and/or are representative of. As I will note, these formal methods 
must undergo significant transformations in the construction of practical computer simulations, which generally result in a detachment of simulations from formal principles and introduces practical constraints that demand independent epistemic assessments as to their reliability. In other words, implementing the formal methods that underlie some computer simulations makes it so that these formal methods are no longer the only epistemically relevant constituents of a computer simulation. Rather, they are but one of the many considerations required to understand what a computer simulation is, how it works and when we can/should trust it in scientific contexts. Furthermore, this detachment implies that the computer simulations are not, strictly speaking, identical to the formal methods from which their construction is derived. As I will argue in detail in this dissertation, if they were, we would not need them. Understanding them and treating them purely as formal methods, or as not significantly distinct derivative processes, risks missing the broader, and more accurate, picture of their functioning and their role in scientific inquiry.

The acknowledgement of extra-formal elements - that is, non-mathematical, non-logical, nonstrictly-theoretical or symbolical elements - as constitutive of computer simulations has moved the current discourse on the role and nature of computer simulations in scientific inquiry towards a broader understanding of them as closer to experimental practice. The assumption being that, if they are not like their formal constituents, if they have other empirically relevant aspects, then they must be closer to the other branch of scientific inquiry: namely, experiments. Hence, throughout the first decade of the $21^{\text {st }}$ century researchers have sought to understand computer simulations in light of their similarities and dissimilarities to empirical experiments. Some researchers sought to equate the epistemic status of computer simulations to that of conventional 
empirical experiments in virtue of the fact, they argued, that empirical—previously unknownknowledge about the subject of inquiry can be attained through the simulation process. Some sought to exclude them as experiments due to their lack of causal intervention and lack of access to material aspects of the phenomenon under inquiry. Others tried to tie their necessary material implementation to an a posteriori status and hence to their status as something closer to experiments. However, more recent accounts within the broader view of computer simulations as experiments have reached interesting conclusions working under the assumption that computer simulations are, if not a conventionally understood empirical practice, then at least a special kind of experiment. One whose main interventions focus on and whose results derive from the manipulation of numerical values and metrics rather than the manipulation of physical phenomena itself.

Both approaches - simulations as formal methods and simulations as experiments- have contributed greatly to the understanding of computer simulations and their relevant constitutive and epistemic elements: models and equations are indeed at the core of many simulation efforts; experimental aims and empirical knowledge are often the driving force behind computer simulations. Understanding both as relevant epistemic elements of computer simulations is important for understanding what computer simulations are and what they do in scientific inquiry. What these approaches miss, however, is the fact that computer simulations are neither formal methods nor experiments in and of themselves. Nor are they closer to one or the other. Rather, as I will argue, they are instruments — technical artifacts — and like other instruments, computer simulations do not fit neatly into either the category of formal methods or the category of empirical experiment because they are neither. 
The status, place and role of computer simulations as scientific instruments, however, is far from obvious. This is particularly due to their narrow applications in their early development as electromechanical extensions of formal analysis and solutions. But it is also due to the fact that instrument seldom figure as an independent and relevant branch of inquiry in the philosophy of science. Rather, arguments must be mustered as to how they fit this category, how they manifest their nature as instruments through the way they function in scientific inquiry and how we can sanction and rely on them, as such. This is the main goal of this dissertation. I suggest that they are technical artifacts whose functioning is distinct from that of the formal methods they emulate and of the formal content they manipulate. I suggest that they are also functionally distinct from the experiments in which they are involved. That is, they do things that neither of these elements of inquiry can do. They are, in short, the things — the technical artifacts, the instruments — with which both formal analysis and experiments can be enhanced and/or carried out. That they are instruments, however, does not settle the matter concerning their role and status in scientific inquiry, rather this fact is but the starting point. Computer simulations are also distinct from other instruments in significant ways for even within this category they elicit a form of hybridity that makes sorting them into their proper place a non-trivial manner. It is in this sense that computer simulations can be understood as novel additions to scientific inquiry. Not, as some suggest, as a sui generis way of doing science but as a special kind of instrument which brings the relationship between theoretical and empirical elements of inquiry to the fore in ways other instruments have not. 
In this dissertation I will also consider an important objection to my view of computer simulations as an instrument. This objection comes from recent developments in the philosophy of computer simulation and takes the form of an argument - which I call the 'argument from heterogeneity' — in which it is implied that given the many and different stages required to construct a computer simulation, the many and different fields of expertise involved in their construction, the many and different components that constitute them, as well as the many and different domains in which computer simulations contribute a significant disruption, they cannot be understood through such a limited concept as 'tool' or 'instrument'. Rather, the argument goes, computer simulations ought to be understood as a practice akin to engineering or medicine, a separate heterogeneous set of skills, aims, communities, etc., that together may well constitute a novel way of doing science. In this light, the notion of 'instrument' seems particularly limited. In this dissertation I show that this is not the case and that in fact definitive exemplars of instruments are the products of the same kind of heterogeneity, reach and disruptive power that this camp thinks beyond the reach of such kind of objects. To exclude computer simulations from being understood as instruments solely on the basis of the heterogeneity argument would mean that paradigmatic instances of instruments, such as the telescope, would have to be excluded too. This would be an undesirable consequence of this position. But furthermore, I show that instruments and computer simulations in particular can be heterogeneous in all the ways discussed. In short, I show that the argument from heterogeneity is not a sufficiently strong argument to dismiss the instrument view of computer simulations.

As I will explain in detail below, the shift towards understanding computer simulations as more closely related to experiments and away from seeing them as mere extensions to formal methods 
has in the last few years gone beyond making direct analogies and comparisons to experiments themselves. Early in the move in this direction, the main strategy philosophers used was to provide examples in which computer simulations could function like experiments themselves and make the case that computer simulations could in fact provide empirical knowledge of the subject of inquiry. Besides the obvious objections from those that consider them mere extensions to formal a priori methods, one of the main obstacles to this view of computer simulations is the fact that computer simulations do not seem to have direct material interactions with the phenomenon of interest. So, philosophers would point to the necessary material implementation of computer simulations as well as to the similarity relation between the simulation specifications, its mathematical structure and that of the phenomenon of interest in order to satisfy this condition in relation to similarity. As I will argue in this dissertation, what is wrong with these strategies, however, is that in their own way they each fail to properly capture the artifactual nature of computer simulations. As I will show here, while it is true that computer simulations are not mere extensions of formal methods and it is true that the work they do is done with the essential inclusion of extra-formal (mathematical, theoretical, logical, etc.) elements, this is not because they are like broader experimental practices, but rather because they are instruments: technical artifacts designed, developed and deployed in scientific inquiry.

Finally, I argue that understanding computer simulations as instruments has significant repercussions for the epistemology of computer simulations. In particular, I argue that — contrary to recent approaches in the epistemology of computer simulations - computer simulations, like other instruments, cannot be deemed trustworthy merely on the basis of non-evidential warrants such as epistemic entitlements - epistemic warrants which give one the right to hold a belief to 
be true without thorough epistemic diligence in the absence of reasonable doubt. Epistemic entitlements are the kind of epistemic warrants that are deployed to ground the pragmatic constraints and considerations in every day epistemic practices. One may say for example, that we are entitled to believe the person driving on the intersecting road ahead of us will stop for their turn if the light is red for them and green for us. We are entitled to expect this without knowing much about that particular person, their car, or that specific stoplight. If there was an accident involving a car that ran a red light in this context and someone asked us why did we chose to accelerate upon our turn on a green light, we can reasonable say that we believed the car whose light was red would stop. Without an appeal to evidence to support our belief, we can reasonably state that we were entitled to believe that they would stop. This is particularly so in the case of absence of evidence to the contrary. If we saw erratic behavior from the incoming car, if we knew they were coming out of a bar, if there was a detectable speed factor that would provide evidence to the contrary of the assumption, then this entitlement would be broken. The gist of this epistemic position is that in the absence of such evidence, we are not to burden the epistemic agent with any further justificatory epistemic requirements such as evidential warrants (explicit justification, proof, empirical access, etc.) to back up their reliance on the way stoplights and drivers are supposed to work at an intersection.

In this dissertation I argue that Burge-style epistemic entitlements such as the ones described above have never been adequate in the inclusion of technical artifacts into the epistemically elevated status of scientific instruments. I conclude this dissertation by contending that in the case of our use and our justification for our use of computer simulations in scientific inquiry, we must distinguish our own reasons for accepting scientific claims/results/artifacts from the kinds 
of considerations that a group of oracle worshippers would deploy as they themselves attempt to justify the use of their similarly epistemically opaque device. The superior epistemic norms of scientific inquiry must be upheld as we consider the status of computer simulations. As an instrument, rather than an experiment, a practice, or a method, computer simulations must be assessed against such demanding norms and standards. Computer simulations can serve as scientific instruments only in those circumstances in which they are designed, developed and deployed in adherence to strong theoretical principles, in which properly curated data is used for their implementation and where proper empirical evidence backs their results. Hence, their widespread usage may not be as easily justified. 


\section{Acknowledgments}

I owe my deepest gratitude to my friend and mentor Professor John Symons. Without his infinitely kind, thoughtful and always inspiring encouragement and guidance, this work would not have happened. 


\section{Table of Contents}

1 Introduction: a review of the debate and the arguments ............................................. 1

1.1 Philosophical reflections on computer simulations and the contemporary debate..........5

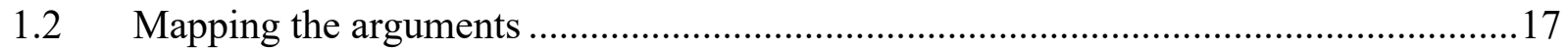

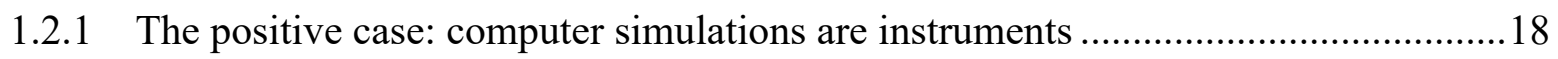

1.2.2 An objection: considerations from the 'practice; view of computer simulations......20

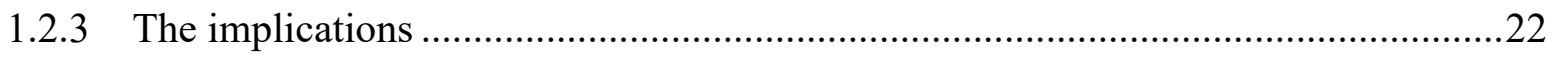

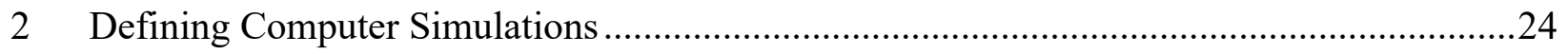

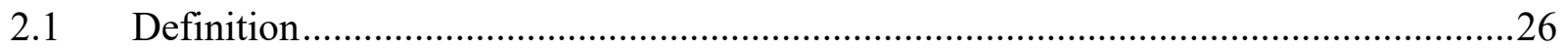

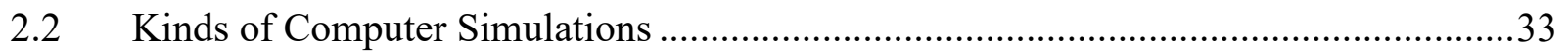

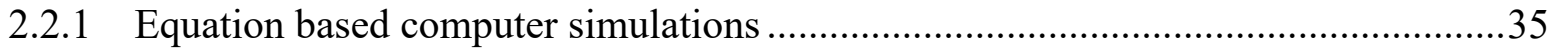

2.2.2 Agent-based simulations........................................................................ 41

2.2.3 Supercomputing and Computer Simulations ..................................................43

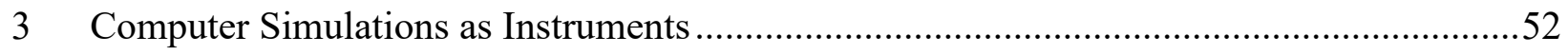

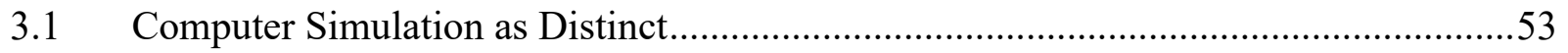

3.1.1 Computer simulations as functionally distinct ..........................................60

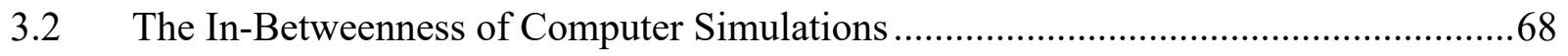

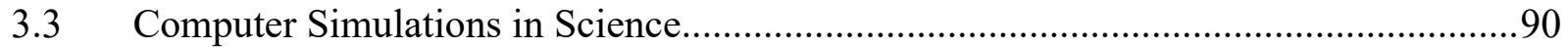

Instrument Taxonomies and Computer Simulations .............................................95

3.5 Concluding remarks on computer simulations as instruments ............................... 107

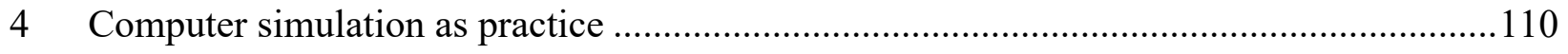

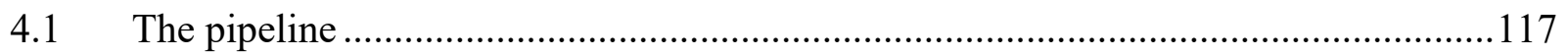

4.2 Practice View vs. the Instruments View of Computer Simulations .......................... 124 


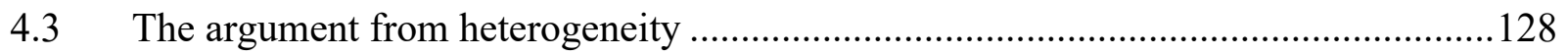

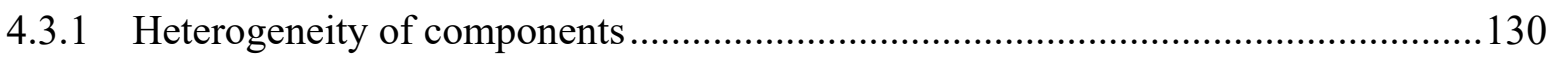

4.3.2 Heterogeneity of practices, methods and communities .........................................135

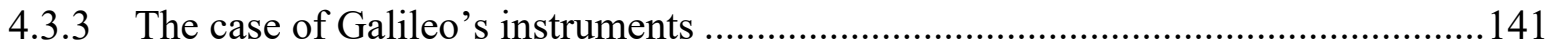

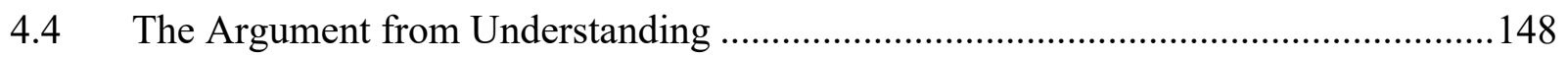

4.5 The Argument from Depth and Reach .................................................................162

4.6 Carving out simulations and making simulation instruments count.............................164

5 Implications: Computer Simulations and Epistemic Trust................................................174

5.1 Epistemic entitlements and computer simulations ................................................176

5.2 Transparent Conveyers, Expert Testimony and Computer Simulations ......................187

5.2.1 Computer Simulations are Not Transparent Conveyers ..........................................188

5.2.2 Computer Simulations are not themselves sources of expert testimony .................205

5.3 Scientific Inquiry, Everyday Epistemic Practices and Computer Simulations ...........209

5.3.1 Higher Epistemic Standards in Science.............................................................214

5.4 Pragmatism as Inadequate Epistemic Framework for Computer Simulations .............218

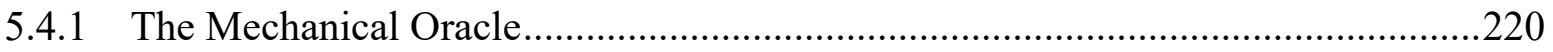

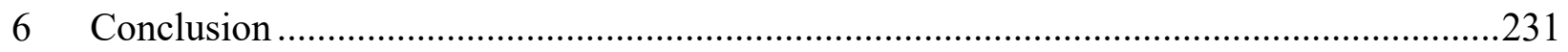

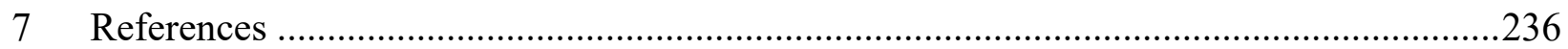




\section{List of Figures}

Figure 1 Two different simulation pipelines (Resch, 2013; 2017 (left); Winsberg, 2010 (right))

Figure 2 Iterated transformation in Ulam's 1967 procedures for 2D cellular automata (Wolfram,

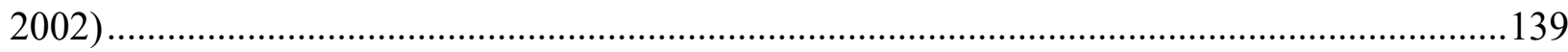




\section{Introduction: a review of the debate and the arguments}

\section{Summary}

Computer simulations began as mechanical solvers for difficult mathematical problems. This provided a framework for philosophers to initially understand them as extensions of formal methods and as having purely formal properties similar to those of the mathematical content they manipulate. The motley nature of their implementation and their role in scientific inquiry, however, was eventually taken by some to be a significant-enough feature to warrant a departure from this early understanding of computer simulations. A new interpretation emerged that characterized them as more closely related to experiments than to models. Here, I argue that computer simulations are neither like models nor like experiments. Rather, they are the things with which both models and experiments can be carried out. They are, I argue, more like instruments than anything else. In this introduction I provide a brief overview of the views described above as well as a summary of the arguments and chapters that will make the core of the rest of the dissertation.

\section{Introduction}

Computer simulations are in widespread use and are often indispensable in contemporary scientific practice. From understanding the formation of galaxies to predicting the path of a hurricane or the population growth of an endangered species, computer simulations provide us 
with novel and important insights. ${ }^{1}$ Computer simulations are also used in the social sciences

from economics to anthropology. ${ }^{2}$ Furthermore, they are also playing an increasingly substantial role in political and corporate decision-making processes. Computer simulations are now commonly used to analyze and predict the development of human-centered systems and often serve as the motivators of policy interventions regarding political, economic and environmental concerns. $^{3}$

Furthermore, computer simulations are used in many settings and often serve a variety of capacities within the same setting. In scientific inquiry, for example computer simulations can be used to understand the development of a system that is otherwise intractable due to its complexity. ${ }^{4}$ They can also be used to elucidate possible underlying mechanisms by which a given behavior may come about. ${ }^{5}$ This can be achieved by using computer simulations to run on multiple parameters and with different data in order to conduct exploratory analysis of possible hypotheses that can in turn guide future inquiry. In industry and other pragmatic settings, such as

${ }^{1}$ See Norton and Suppe (2001) for a thorough overview of the role of computer simulations in climate science.

${ }^{2}$ See the work of Nicole J. Saam (1999; 2009; 2017a; 2017b) for many examples of the role of computer simulation in the social sciences.

${ }^{3}$ See Naomi Oreskes for a thorough analysis of the role of computer modeling in scientific inquiry, particularly in earth sciences (1994) and the implications of modeling practices in policy related to tobacco deaths and climate change (2011). Eric Winsberg's (2018) Philosophy and Climate Science offers a thorough review of the epistemic and historic challenges of computer simulations in sciences that address climate change.

${ }^{4}$ As an example of this complexity, consider the number and degree of complication of calculations inherent to dynamic equations such the Navier-Stokes equations, which quickly become something that cannot be done/followed by hand. That is, tracing both the processes and the outcomes of the dynamic features of such equations is intractable.

${ }^{5}$ Nariya et al., (2016) for example, explore — through a computational biology framework — the way in which particular kinds of bacteria develop sufficient protein to form a propeller tail that allows it to move. 
disaster response, computer simulations are used to forecast possible states of a system that we are concerned with. Mapping out possible trajectories of a storm system via computer simulation, for example, is a critical part of how emergency responders strategize and prepare. Across a range of enterprises and institutions computer simulations are deployed for assessing risks and opportunities. ${ }^{6}$

In spite of their widespread use, our understanding of the epistemic status of computer simulations in inquiry is far from settled. That is, whether, how and when we can trust them as yielding scientifically reliable results is something that has generated much philosophical debate (Barberousse and Vorms, 2014; Hubig and Kaminski, 2017; Symons and Alvarado, 2019). They are, after all, a fairly new addition to human inquiry (Fox-Keller, 2003; Durán, 2018; Winsberg, 2019). Most significantly, there is continuing debate concerning their role in science given that their trustworthiness and reliability are far from settled in some relevant fields (Symons and Alvarado, 2019). Playing a central role in these debates are questions about what computer simulations actually are — whether they are models or experiments, for example_-, what their place in the scientific landscape is, and what role they play in the acquisition of scientific understanding, if they can play one at all (Symons and Boschetti, 2013).

\footnotetext{
${ }^{6}$ An important distinction between one setting (science) and the other one (industry) is the degree of relevance that explanations have. While forecasting the future consumption habits of costumers is important for revenue considerations, the underlying mechanism by which they fluctuate may not be as relevant. Similarly, in medical settings whether something is more likely to cure a deadly illness may prove to be more important than knowing why or how it does so. In strictly scientific settings however, or in the long-term interest of knowledge acquisition practices, explanation matters (Symons and Alvarado, 2019).
} 
In this dissertation, I argue that computer simulations are technical artifacts deployed as instruments in scientific inquiry and that they ought to be treated, understood and sanctioned as such. Treating simulations as scientific instruments is a departure from existing positions in the philosophical literature on this topic. I will elaborate and defend a definition of computer simulations as instruments, I will show how this view is preferable to the positions in the existing literature, and I will explain how treating them as instruments helps us answer some central questions in the epistemology of computer simulation. I will especially focus on questions concerning the kinds of epistemic warrants that can justify their use.

In addition to my purely conceptual arguments, I will examine some examples of the role that scientific instruments have played in the history of science. Some of the episodes I discuss, notably the example of Galileo's process of sanctioning his telescope as a scientifically reliable instrument, are illuminating for our current scientific context, in which the question of how we can come to trust computer simulation is of central importance. In particular, these historical examples elucidate the fact that artifacts such as instruments have a significant and independent role in shaping standards, practices and scientific inquiry itself. This independent importance of instruments emerges together with the many social and historical factors contributing to their ultimate acceptance as reliable. These historical examples should lead us to challenge conventional views of computer simulations. ${ }^{7}$ Specifically, these examples encourage us to

\footnotetext{
${ }^{7}$ It is important to note at this point that my use of examples from the history of science does not constitute a positive argument that proves computer simulations are instruments. Rather, these historical cases are deployed as a defense, in the form of a counterexample, against the views that suggest that the term 'instrument' and the conceptual framework surrounding it is inadequate to capture the rich and complex heterogeneity of computer
} 
reconsider the conventional view that the many aspects of science modified by computer simulations, the many elements and components that constitute a computer simulation, and the many areas of expertise required to create and deploy a computer simulations preclude us from understanding them as tools or devices (Resch, Gehring, and Kaminski, 2017). My account will help to unify the diverse range of topics within the debate around computer simulations.

\subsection{Philosophical reflections on computer simulations and the contemporary debate}

Understanding computer simulations as instruments contrasts sharply with prevailing philosophical views. Such views of computer simulations are situated in a landscape of positions that is marked by two major reference points: either computer simulations are like models, or they are like experiments. The literature on scientific models has shaped the discussion of computer simulations in ways that I believe are unhelpful. These reference points are inherited from older debates concerning the nature of models in philosophy of science. ${ }^{8}$ This is particularly the case for philosophers like Frigg and Reiss (2009) who questioned the

simulations. As I will argue in detail througout chapter 4, other instruments in the history of science have had similarly rich and heterogeneous developments. Hence, the heterogeneity and the depth of disruption to scientific inquiry commonly associated with computer simulations are not sufficient reasons to exclude them from being understood as scientific instruments rather than as broad experimental practices.

${ }^{8}$ See Frigg and Reiss' (2009) response to Humphreys' (2009) to see how the suggested philosophical novelty of computer simulations in science was dismissed on the basis of existing work on the philosophy of scientific models. Morgan and Morison, (2009) similarly make the case that computational methods such as computer simulations could be understood with a similar framework of 'models as mediators', Weineberg (2012) and Pincock (2011), respectively, briefly deal with computer simulations by also treating them as special cases of mathematical/scientific modeling. 
philosophical novelty of computer simulations. However, the influence of such a position can be found in all sides of the contemporary philosophical debate on computer simulations. There are views that interpret the formal aspect of computer simulation as direct implementations of models (Hartman, 1996) and/or that interpret them only as special kinds of models (Weinberg, 2012). There are also views that stress the motley aspect of computer simulations and their components - broader accounts of computer simulations - but that continue to import concepts from the philosophy of models to deal with the representative aspects of computer simulations. That is, because both models and simulations represent, they continue to be treated as roughly a special kind of modeling. Because of the influence of these debates, frequently in the philosophical discussion of computer simulation, one finds a dichotomy between the following positions. On one side of the debate, computer simulations are seen as belonging to the abstract realm of elements of inquiry, such as mathematical/theoretical models. In so far as computer simulations are used to provide solutions to mathematical models such as equations, or to represent dynamic processes that are specified by theoretical principles and assumptions, then their content and operations can be taken to be just extensions of these formal elements of inquiry (Dalmedico, 2001) $)^{9}$. The other side of the debate, as I will show in this section below, is a direct response to the position just described. This second side of the debate maintains that computer simulations are seldom a direct result of formal methods and that many additional non-

\footnotetext{
${ }^{9}$ Weinberg (2012) and Pincock (2011), for example, both concerned with the nature of modelling as scientific representation, take computer models to be merely yet another formal extension of mathematical representation. Neither takes into account their necessary implementation as an epistemically relevant distinguishing feature. As such, computer simulations are treated merely as special cases of mathematical modelling and treated as purely formal abstractions.
} 
theoretical and non-mathematical factors come in to play when running a simulation successfully. Under this view, computer simulations are not merely model solvers. Those that advocate an understanding of computer simulations as 'something more than' formal also seek to provide a view of computer simulations as an element of scientific inquiry that is capable of generating new insights about the system being simulated. That is, computer simulations, under this view, are capable of yielding information beyond what is contained by the assumptions and input of a mathematical or theoretical model (Parker, 2003; Barberousse et al., 2009; Ruphy, 2015).

Therefore, a division appears between those that inherited an understanding of computer simulations as a machine-implemented continuation of formal methods, and those that respond to this position by suggesting that we should position computer simulations on a par-or close toempirical practices such as scientific experiments.

Hence, philosophers of computer simulation (See Durán, 2018; Winsberg, 2019) identify two different viewpoints on computer simulations: the narrow and the broad views of simulation. According to this understanding, there are those that see computer simulations as problemsolving techniques and those who see them as the practice of description of patterns of behavior in a system. The functions a computer simulation is expected to fulfill, and therefore the role they are expected to play in scientific inquiry provides the dual framework for these two 
categories. ${ }^{10}$ For some, computer simulations are a set of problem-solving techniques (Durán, 2018 p. 9). They are just model and equation solvers. To others computer simulations are a new form of experiment that encompasses broad and heterogeneous set of practices (Winsberg, 2010; Gehring, 2017; Resch et al., 2017). In this latter view, computer simulations are broadly defined as experiments (Barberousse et al., 2009; Lenhard, 2004; Parker, 2009).

As I briefly mentioned above, the first approach emphasizes the role of computer simulations in providing solutions to modeling efforts; the second approach emphasizes the representational role of computer simulations. This distinction also maps on to the overall reasons to use computer simulations in the first place. Those that take them to be problem-solving techniques only see their use justified when the problems that they are solving do not allow a conventional approach (Humphreys, 2004; 2009). More specifically, the argument goes, the only reason to use computer simulations is if the problem that requires to be solved does not allow for analytic, tractable solutions. This is the narrow view of computer simulations (Winsberg, 2019). On the other hand, those that take the representational features of computer simulations to be their most important feature can — or so the argument goes — justify their use in settings in which other extra mathematical concerns are of interest and /or can be said to provide additional insight into a phenomenon of interest. This is the broad view of computer simulations.

\footnotetext{
${ }^{10}$ Symons and Boschetti $(2011,2013)$ depart from these positions. They offered a formal argument to the effect that the primary scientific function and virtue of computational models is predictive. This is due to irreversibility in computational systems, meaning that computational models can generally arrive at the same state via many possible sequences of previous states. While physical states have a unique history, representations of those states in a computational model will usually be compatible with more than one possible history in the model.
} 
It is evident, for example, that when we are dealing with the multiple and diverse components of computer simulations, the implementation of the formal manipulations therein and their results, there is something more than mere calculation or formal abstraction. ${ }^{11}$ Even views such as Hartmann's - whose narrow definition of computer simulation has served for decades as the starting point for any reductionist ${ }^{12}$ account of computer simulation — have had to acknowledge early on in the debates that simulations as scientific practice also have multiple functions: they can be seen as techniques, heuristic tools (2005 p.9) for experimenters, substitute experiments, etc. That is, For Hartmann, computer simulations as techniques can be understood as a series of methodological procedures followed by practitioners; as heuristic tools they can offer representational insight towards future inquiry, and computer simulations can also act as a substitute experiment ${ }^{13}$ in cases where both the understanding of a phenomena as well as the data are closely related to numerical values ${ }^{14}$ or cases in which the only access to the

\footnotetext{
${ }^{11}$ As I will argue in the following section, at the very least, it is evident that computer simulations are distinct from their constituent processes and/or components in that, even when they do the things that other elements of inquiry do, they do them differently: faster, better, etc.

${ }^{12}$ These are the sets of views encompassed by what Winsberg (2019) deems 'narrow' and Durán (2018) calls 'problem solver' and which deem computer simulations as similar in nature to mathematical solvers.

${ }^{13}$ i.e. experiments in silico, a phrase coined to denote experimental practices done through circuitry and digital methods.

${ }^{14}$ It is a deep epistemic and metaphysical question — not to mention beyond the scope of this dissertation - the extent and degree to which our understanding of the world (theoretical, mathematical, etc.) in fact corresponds and how it could correspond to how the world actually is. This question is beyond the scope of this dissertation.
} 
phenomena is through indirect yet quantifiable factors (i.e. astronomical phenomena, etc. $)^{15}$.

This more nuanced account of computer simulations is able to accommodate some of the motley features briefly discussed above. This account also points to a more complex view of computational methods than the purely mathematical view.

Some within this latter camp have come to view computer simulations more like enriched implemented models. That is, they see them as methods that can provide insight to theoretical principles by providing, through trial and error, explicit (algorithmic) accounts of the possible dynamics - functions - that guide the transformation of quantitative inputs. Under this latter view, computational methods such as computer simulations, like models, aren't solely the result of simple arithmetic operations. Besides addition and subtraction, they can also encompass more sophisticated operations related to calculus and statistical analysis. This interpretation moves the balance towards a richer understanding of computer simulations and gets the discussion away from their simplistic characterization as mere "number crunchers" while still maintaining their fundamental formal character.

On the other side of the debate, the side of those that advocate for a broader definition of computer simulation, several positions arose (Lenhard, 2004; Barberousse et al., 2007; Morrison,

${ }^{15}$ This is, as I will show below, the position held by Margaret Morrison on the relation between computer simulations and experimental practice. Her view, I will argue is ultimately a deflationary view of experiments rather than a inflationary account of the status of computer simulations as empirical practices. 
$2009 ; 2015)^{16}$ that take computer simulations and the practices associated with them to be more akin to scientific experimentation (Ruphy, 2015). They argue that in scientific practice computer simulations include extra-mathematical considerations, methods and processes (Winsberg, 2010). Although computer simulations often include, use or are constituted by the numerical tools exemplified in their origins they are few steps removed from these first uses.

Advocates of this view (Barberousse, et al, 2009; Morrison, 2009; Winsberg, 2010) make of simulations more of a patchwork of diverse processes by which we seek to recreate quantifiable states of a system (Winsberg, 2003). These diverse processes often include measurement practices (Morrison, 2009), representational features (Barberousse et al., 2009), and developing and testing hypotheses and theories (Hartmann, 2005). Winsberg, for example, tells us that in this broad view computer simulations are more like "comprehensive methods to study systems" (Winsberg, 2010). ${ }^{17}$

Winsberg also believes that these methods, through a series of transformations by motley processes, can be/have to be implemented. This implementation process includes choosing a model, finding a way of implementing that model in a form that can be run on a computer,

\footnotetext{
${ }^{16}$ Although Morrison is a defender of the materiality thesis, her approach is unconventional. She is different from Parker's view and Winsberg's view in that the materiality of computer simulations is located in the relationship "between the simulation model and the system it represents." (Morrison, 2015 p.221) Thus, her view of the materiality thesis does not involve the implementing machine.

${ }^{17}$ This move alone, to call them methods, already signals a drive towards an even broader conceptualization of computer simulations beyond that of a simple experiment. That is, if we follow this last step, computer simulations are no longer just experiments but rather methods by which experimentation can take place. I will have to say more on this in chapter 4.
} 
calculating the output of the algorithm, as well as visualizing and studying the resultant data, amongst other things. According to this view, the method of computer simulations encompasses this entire process — used to make inferences about the target system that one tries to model—as well as the procedures used to sanction those inferences (Winsberg, 2019). He calls these processes 'simulation studies' (Winsberg, 2003) and describes them the following way:

"Successful simulation studies do more than compute numbers. They make use of a variety of techniques to draw inferences from these numbers. Simulations make creative use of calculational techniques that can only be motivated extra-mathematically and extratheoretically." (Winsberg, 2019).

By taking into account these extra elements of the practice of simulation, Winsberg sheds light on the more complex aspects of computer simulations. Just like experiments, each computer simulation is different from the other and its epistemic import must be independently assessed (Barberousse and Vorms, 2014). What is meant by experiment here, however, is not exactly clear and this lack of clarity has been the subject of much debate in the literature of computer simulations. (Lenhard, 2007, Barberousse, 2007)

So, here we have two views of computer simulations: as formal methods and as experiments. It is important to note, however, that the debate doesn't stop with the simple dichotomy between simulations as models and simulations as experiments. As noted a couple of paragraphs above, some of those that acknowledge that the implementation stage of computer simulations is difficult enough (as well as significantly distinct from other stages) to change the justificatory 
basis for our reliance on their results, see computer simulations as broadly experimental engineering practices, a motley process of makeshift consensus (Gransche, 2017). These practices and processes do not amount to a simple analogy to conventional experiments. Rather, the concept is even broader. Their implementation, of course, requires experimentation and the gathering of supporting data, or even metadata — on both, the implementation itself and on its results - to justify our reliance on it (Von Neumann and Brucks, 1946; van de Velde, 1960; Simon, 1969; Metropolis, 1987; Fox-Keller, 2002; Lenhard, 2012, Winsberg, 2010; Morrison, 2015; Kaminski, 2017). But this also means that experts in diverse communities without a common language must learn to communicate as they work together without a unifying framework (Gehring, 2017). These views are a direct response to the narrow understanding of computer simulations as extensions of formal methods. But they are also a response to those views that try to understand them as experiments. To those on this side of the debate, computer simulations are something even broader: they are a practice..$^{18}$

What all these views — narrow, broad, and broader — are missing is the fact that the actual use and development of computational methods in scientific practice does not fit their interpretations as abstract objects or as experiments. Rather, as I will argue in detail, computer simulations are designed, developed and deployed as the kinds of artifacts that are better understood as instruments. Hence, neither the view that places computer simulations as closely related to mathematical models, nor the one that places them, in an attempt to reconcile their unique

\footnotetext{
${ }^{18}$ This view of computer simulations will get a thorough and independent analysis in chapter 4 .
} 
contributions to inquiry, as closely related to experiment are adequate to capture their use, let alone their nature. And neither of those two views would want to categorize them in such a broad concept as a practice. Computer simulations are not like engineering, they are the product of engineering. They are also not like medicine because, even intuitively, one can see that computer simulations are closer to devices used in medicine than they are to the practice as a whole. ${ }^{19}$

What is clear from the complexity and size of contemporary scientific projects such as those involving the creation of a computer simulation, is that these instruments, like many modern instruments, often encapsulate multiple components and various functions all at once (Simoulin, 2017). ${ }^{20}$ For example, a weather prediction model simulation is a simulation whose expected function is, of course, to allow insight into the behavioral patterns of a system: namely the weather. It does so by solving equations in an amalgam of models in a way and with a speed that would be nearly impossible for any one human to do analytically. Furthermore, a weather prediction model simulation also provides insight into future states of a system that would very likely be inaccessible to us otherwise. Hence computer simulations such as those used for weather prediction provide access to the dynamic behavior of a phenomenon, solve equations, represent the inferences of models, and through expert interpretation, provide access to future states of a system. Often, the most important element of the simulation besides their computational aspect is the integration of information in an intelligible form: visual or otherwise.

${ }^{19}$ The use of an intuitive notion here is not to serve as argument in itself. Rather, it is deployed merely as a starting point towards a motivated argument for which I will provide details in Chapter 3.

${ }^{20}$ I will provide full details on this line of argumentation in chapters 3 and 4. 
As such, it is unclear whether they are captured by either the narrow or the broad view alone. This is because the narrow view of computer simulations sees in them nothing else than mathematical procedures without taking into account the integrating efforts that make the interpretability of results possible. In other words, without the many 'stitching' steps in between models, equation and solutions at different scales and of distinct metrics and phenomena there would be no unified representational product to interpret as a simulation of the target system. On the other hand, the broad view of computer simulations fails to capture the fact that despite there being many heterogenous elements - at the component level as well as at the procedural levelcomputer simulations can still count as a single unified representational device. In short, both views miss the fact that a computer simulation, as complex as it may be, may still be understood as the instrument with which the many functions described above are carried out. The same can be said about those views that characterize them as a practice. They all simply miss the thing they are in fact pointing at.

In fact, as I will argue in sections 3.4 and 4.3.1, computer simulations are hybrid instruments. They are composite structures of computational devices, they carry out multiple operations through multiple constituent components and they cross lines in different taxonomies of laboratory instruments: as I will argue in detail in section 3.4, computer simulations are hybrid epistemic enhancers (Humphreys, 2004). They are hybrid in that they are often both capable of simulating the processes by which an experiment is conducted (Barberousse, 2019) as well as 
acting as measuring devices ${ }^{21}$; computer simulations are also hybrid in that they are able to produce (simulate) a phenomenon in an environment that does not exist in nature as well as of modifying the (simulated) behavior of a system through value-intervention (Morrison, 2015). That is, as I will explain, computer simulations, in so far as they manipulate many of the possible values of input data, meet Margaret Morrison's — deflationary — criteria of what amounts to an experiment: they are able to both recreate a phenomenon not found in nature and simultaneously able to modify the — simulated (in so far as only values are being manipulated) — behavior of a system. However, even when computer simulations meet the criteria set forth by Morrison to call them experiments, what we find is that in fact computer simulations are not the experiment itself but rather the thing with which the experiment is conducted, the artifact with which those values are manipulated. In short, what we find is that the computer simulation is the instrument with which scientific inquiry is carried out.

Because of this, treating computer simulations in either the narrow sense as mere solvers or the broad sense as vaguely defined experimental practices yields limited insight into their nature. Whether one is referring to agent-based or equation-based simulations or whether one has a broad or a narrow view of computer simulations, what matters is that they are the instruments with which the dynamics of equations or agents are carried out, with which measurements are made (Morrison, 2015), and/or with which experimental set ups are implemented (Barberousse, 2019). What is more informative in elucidating the epistemic role and import of computer

\footnotetext{
${ }^{21}$ In sections below I will say more about this, particularly as it pertains to Morrison's (2015) thorough discussion of computer simulations as measuring devices.
} 
simulations in scientific inquiry is to investigate what kind of instruments they are in virtue of what they do for us in inquiry and how they achieve what they are supposed to do, which I will do in this dissertation, particularly in chapter 3 and 5.

While it is true that computer simulations can implement scientific models and it is also true that computer simulations involve and realize the many problem-solving techniques often ascribed to them, computer simulations are not the experimental techniques they carry out nor are they the models they implement. They are the instruments with which the tasks of experimentation and modeling are executed. In this dissertation, I will show that this shift in perspective can provide a fruitful new way of looking at the epistemology of computer simulation. While it is obviously true that computer simulations involve hardware and software and that these are human artifacts of a particular kind, by understanding them as scientific instruments, I show how to move beyond the dichotomy of modeling and experimentation that has dominated the debate to date.

\subsection{Mapping the arguments}

In developing my defense of the instruments view, it became clear that I need to show why we need to move beyond an inherited dialectical dichotomy (Ruphy, 2015 p.133) between those who view computer simulations as mere subsets of formal abstract methods and those that advocate for a broader definition of computer simulations. As the details of my definition of computer simulation unfold, I will argue that, through this conventional dichotomy, conventional views about the role and epistemic import of computer simulations have also inherited an important blind spot: they have unreasonably neglected the role of instruments in scientific inquiry. 
Because of this omission, the current philosophical literature on computer simulations has been caught in a dichotomy that guides much of its development. The aim of this dissertation is to provide a third way out. In the sections below I lay out, briefly, the three overall argumentative trajectories that will make the case for this alternative position: 1 . the positive case of computer simulations as distinct technical artifacts, 2. the inadequacy of the objections to my view from the 'practice' view of computer simulations, and 3. the epistemic implications for the way in which we rely and trust on computer simulations once we understand them as scientific instruments.

\subsubsection{The positive case: computer simulations are instruments}

In this dissertation I will show that computer simulations are instruments. The first series of arguments towards an instrument view of computer simulation consists in acknowledging that they are distinct objects from the content they manipulate (formal, symbolic, numeric) and from the conceptual elements from which they are derived (models, theoretical principles, etc.). This argument extends towards their functional distinctiveness as well. That is, computer simulations are not just a distinct object from those things mentioned above, they also have distinct functions from those things and from the broader functions of the experimental settings in which they are deployed. In other words, computer simulations are not the same as the equations they solve, they are not the same as the models they implement, but they also do not do what models or what equations do. At the very least, when they do perform similar functions to other elements of inquiry, they do so in a sufficiently different manner from them: faster, discretely, mechanically, etc. This last point alone suffices to distinguish them from such other elements of inquiry as 
models or equations, but also from experiments. That is, computer simulations also do not do what experiments do. When and if they can be characterized as doing so, they do it differently: virtually, for example. If computer simulations did not do things that models or equations do not, or if they did not do them differently, there would be no use for them. If they did not do something different and/or in different ways from experiments they would not be deployed as ubiquitously as they are in laboratory settings.

As technical artifacts (Kroes and Meijers 2006; Kroes 2009) — as they model, control, represent, and in some cases measure and even demonstrate - computer simulations work via a series of well-articulated specifications at many conceptual and implementation levels. Like other technical and physical artifacts, computer simulations also perform computational and representative functions (Baird, 2004). That is, as instruments, they are capable of carrying out functions that other instruments do. ${ }^{22}$ However, this fact does not simply mean that computer simulations are completely like other instruments or that they ought to be treated (understood, assessed, etc.) without critical scrutiny as such. They are also sufficiently distinct from conventional instruments. A second set of arguments in this dissertation will argue that like other composite instruments (Simoulin, 2017) such as the synchrotron, which is an instrument made of many instruments, computer simulations are the kind of instrument made of many instruments. Because of this and other reasons I will show, in section 3.4, that they are hybrid instruments.

\footnotetext{
${ }^{22}$ Notice here that the similarity is with other instruments and not with other modes of inquiry. In other words, the claim here is narrower than the points made a few sentences before: it pertains strictly to the realm of instruments and not to the realm of experimental practice or formal methodologies.
} 
This explains a recurring observation in the philosophy of computer simulations that characterizes them as not fitting in the category of theoretical endeavors and also not fitting within the category of conventional experimental processes. They are, as many have pointed out, somewhere in between. As I will argue in section 4.2, their hybridity explains this inbetweenness within the context of scientific instrumentation. That is, rather than being sui generis third element of scientific inquiry in between theoretical endeavors and experimental methods, computer simulations are hybrid instruments in between other kinds of instruments. This same fact corroborates the difficulty in placing them within any dichotomous category system, but more precisely, it locates this difficulty within the taxonomies of scientific instruments as well: some instruments measure, some instruments help us detect, some instruments represent, and yet others help us manipulate. As I will show in sections 3.1.1 and 3.4, computer simulations do many of these things, and fail to do some of them. Because of this they remain as something in between and do not fit neatly in any of the categories in conventional taxonomies of laboratory instrumentalia.

\subsubsection{An objection: considerations from the 'practice; view of computer simulations}

As I briefly explained above in section 1.1, on the broad conception of computer simulations there are those that besides acknowledging the extra-formal elements of computer simulations also argue that the term refers to something even broader than just implemented models or experiments. As a result, some on the latter side of the debate understand computer simulations more as a set of experimental practices whose epistemic assessment ought to be construed taking into account sociological features such as community consensus among heterogeneous experts, 
engineering ingenuity, and other social implications surrounding the practitioner's world (Gehring, 2017; Gransche, 2017; Saam, 2017; Durán, 2017; 2018). For this view, computer simulations are, in short, a practice. Those in this camp explicitly reject any analogies to instruments as adequate understandings of computer simulations. In their view, terms such as 'instruments', 'devices', and/or 'tools' are just too limited to understand and capture the broad and complex set of practices associated with computer simulation.

In chapter 4, I will argue directly against the reasoning of those that reject the instrument hypothesis. In particular, I will be arguing against the views, such as those expressed by Resch et al. (2017) but also implied elsewhere (Barberousse, 2019), that suggest computer simulations cannot be understood as devices because of the intricate and heterogeneous processes and communities involved in their creation and use. I will show, through examples in the history of science, that these views, and in particular the arguments that stem from the heterogeneity surrounding computer simulations, fail to support the argument against the interpretation of computer simulations as instruments. Furthermore, I will show that an implication of these views is the denial of the status of 'instrument' even to quintessential exemplars of the concept. That is, if we take heterogeneity of expertise, components and implications, as a basis to deny that something is an instrument, then many of the things we would intuitively deem to be instruments will fail the test. This is because many modern instruments require such heterogeneity to be what they are and do what they do (Simoulin, 2017). This is not only the case for modern instruments, however. The arguments that stem from the practice view also entail that even such uncontroversial cases as the telescope fail to count as instruments. I take this to be a reductio of 
their position against the instrument hypothesis regarding computer simulations.

While examples from the history of science, such as Galileo's telescopes, do not in and of themselves constitute a positive argument towards the case that computer simulations are an instrument, these examples constitute a counterexample to those views that deny the possibility that an instrument can be the product of a heterogeneous set of elements. Furthermore, these examples are also a counterexample to those views that suggest, like the practice view does, that instruments in and of themselves are not capable of significant disruptions to the fabric of scientific inquiry. As I will argue in section 4.5 both the telescope and computer simulations can and have had a broad impact in the way that science is conducted and even in what we take to be science to begin with. More importantly, both are instruments.

\subsubsection{The implications}

Finally, in chapter 5, I will show that understanding computer simulations as instruments is not merely a matter of labels. Rather, the concept of instrument brings along with it conceptual and practical implications for the ways in which we construct a viable epistemology around computer simulations. This is particularly the case when we ask questions about whether or not we have epistemically sound reasons to trust them and what form these reasons take. These questions and some of the answers from the literature of philosophy of computer simulations will be critically assessed in chapter 5 . In particular, I will be arguing that conventional approaches to justifying our reliance on computer simulations through non-evidential warrants called epistemic entitlements is simply inadequate. I will also argue that predictive prowess is not sufficient to 
establish an instrument as scientifically reliable and therefor trustworthy. I will argue that understanding computer simulations as instruments, rather than as practices or as formal methods, elucidates why we cannot trust them via entitlements and why merely pointing to their pragmatic applications and their predictive success is not sound epistemic practice: namely, because that is not the way scientific instruments get to gain their status as scientific. Rather, there is an expectation that the inclusion of technical artifacts in the realm of scientific instruments is guided by stricter normative epistemic requirements and by strong adherence to theoretical principles. Therefore, computer simulations, like other instruments, ought to be sanctioned (or vetted) with this in mind and not through accounts of the epistemic practices in everyday life. ${ }^{23}$

${ }^{23}$ A clarification needs to be done here to avoid a future confusion: throughout this dissertation I note that just like other instruments are sanctioned and/or vetted before they can be fully considered for scientific use, so computer simulations must be too. However, what this does not mean is that the same particular mechanisms by which we vet and/or sanction other instrument will be adequate for and should be used on computer simulations. 


\section{Defining Computer Simulations}

\section{Summary}

In order to begin our assessment of computer simulations as an instrument it will be useful to provide a definition. Although the many challenges of doing so are acknowledged here, this chapter nevertheless does provide a working definition in section 2.1. In this chapter I also contrast my definition with existing ones in the literature of philosophy of computer simulation and further elucidate the many different interpretations of computer simulations throughout the literature. In particular I talk of the kinds of computer simulations relevant to our discussion in section 2.2.

\section{Introduction}

Although it is true, as many have noted (Resch, 2013; Durán, 2018; Resch et al, 2017; Gehring, 2017), that a fully articulated and unified definition of computer simulation is difficult to extract from a survey of the literature across diverse disciplines, an initial common-sense definition can be generated in a fairly straightforward manner when one regards them as instruments. In fact, this is one of the initial benefits of understanding computer simulations as instruments rather than as practices or extensions of formal methods. As with other instruments we can identify computer simulations and their role in inquiry by enumerating their components, the way they 
function, the functions they perform and what they are deployed for. ${ }^{24}$ (Heilbron, 1993; Van Helden and Hankins, 1994 p.4; Harré, 2003; Kroes, 2003 p.68; 2006; 2009 p.53; 2012).

Saying that instruments can be identified in this way and that computer simulations follow suit will prove to be an important distinction to consider as we compare and contrast them to other elements of inquiry such as formal methodology and experimental practices in following sections. While I will not expand on the details of this distinction in this section, I will initially state my point with the following set of broad comparisons: It is much more difficult to pin down, through a definite definition, what engineering is and what engineering does than pointing to what a specific engineering or engineered instrument is or does; it is more difficult to define what those involved in putting together the large hadron collider (LHC) are and do than it is to define what the LHC is and/or does.

A computer simulation of a target phenomenon is to those in charge of putting it together (engineers, computer scientists, graphic designers, etc.), those operating it (engineers, computer scientist, etc.), and those using it (scientists, industry professionals, policy makers, etc.) what a telescope is to those putting it together, operating it and using it. That is, both the telescope and the computer simulation are artifacts that require complex distributed stages in their assembly, operational attention as they carry out their functions and interpretative skills from experts with

\footnotetext{
${ }^{24}$ It is important to note a triadic distinction: the things a thing does, the way it does them and what the thing is used for. These three items in the distinction can and do in fact often overlap, but it isn't always the case. One can imagine, for example that a carburetor mixes air and fuel, it does so through the motions of calibrated valves and it is deployed, mainly, to help combustion in engines. These three things are not one and the same.
} 
vast background knowledge of the phenomenon of interest. Yet neither of these requisite elements is sufficient to define what a computer simulation is or does, just like defining a computer simulation as a distinct artifact will do little to define any of the aforementioned elements.

The conceptual move from understanding computer simulations as practice and/or as method to understanding them as instruments is promising in the same way that defining a telescope by its components and what it does refocuses our attention on the artifact itself rather than on the elements and prerequisites of its construction, development and/or use.

In this chapter I provide a straightforward definition of computer simulation that reflects this fact as well as a broad overview of different kinds of computer simulation along with brief examples of their uses. Doing so will provide the reader with a contextual framework to position the development and use of the computer simulation as a genuine addition to general scientific and/or specific laboratory instrumentalia (Durán, 2018). Furthermore, starting with a straightforward definition serves to provide a clear view of the aim of this dissertation.

\subsection{Definition}

Throughout this dissertation, I will defend and use the following definition of computer simulation: Computer simulations are technical artifacts that are the product of joint work from 
scientists, programmers and engineers ${ }^{25}$ implementing a series of hierarchical procedures (norms and steps of experimental practices), formal processes (logical rules and manipulations), and representational assumptions or hypotheses (models or equations) about the dynamic character of a phenomenon on a variety of electronic devices, which through their diverse yet unified functional processes (seek to) mimic and/or represent the relevant elements of said target phenomenon.

In their most basic form, these electronic devices carry out, in the order prescribed, the necessary computations (the algorithmic processes detailing the required calculations and manipulations of resulting variables' values and their place within its hardware infrastructure) and apply the resulting solutions to the embedded representational dynamics (i.e. the model, the equations, the grids, etc.) associated with a target system in order to provide a comprehensive analysis (visual, or otherwise) of its changes.

A commonly used definition of computer simulations in the relevant literature (Durán, 2018;

Primiero, 2019; Winsberg, 2019) is Paul Humphreys' definition, which goes as follows:

\footnotetext{
${ }^{25}$ Oftentimes this ends up being one and the same person. Although this can sometimes lead to transparency and quality problems due to idiosyncratic incentives, practices and legacies related to science in academia, the issue is not a new one and is not particular to software intensive practices. Traditionally scientists, particularly those involved in groundbreaking research, have often had to put together their own instruments before relying on a standardized equipment industry (Bracegirdle, 1993 p. 295). In this sense, the fact that scientists are now required to code and/or put together their own computer simulations is not a significant deviation from traditional historical practice.
} 
A system $\mathrm{S}$ serves as a computer simulation of an object or process B just in case $\mathrm{S}$ is a concrete computational device ${ }^{26}$ that produces, via temporal process, solutions to a model that correctly represents B, either dynamically or statically. If in addition the computational model used by $\mathrm{S}$ correctly represents the structure of the real system $\mathrm{R}$, then $\mathrm{S}$ provides a core simulation of system R with respect to B. (2004, p.110)

Several of the success and reference terms in this definition are worth unpacking in detail. There is a great deal of work to be done in order to understand what it means for a computational model to 'correctly represent' for example. While it is true that some degree and kind of similarity is the aim of simulation in general (Weisberg, 2012) there must be an explicit acknowledgment of the role of idealization in scientific representation (Humphreys, 2009b; Pincock, 2011). Sometimes these simulations are successful in accurately representing a realworld system; sometimes they are successfully based on theoretically coherent dynamics; sometimes computer simulations are about other artificial systems, including computational

\footnotetext{
${ }^{26}$ It is important to note here that the naming of $\mathrm{S}$ as a concrete computational device strongly resonates with my aim to understand computer simulations as technical artifacts. However, what I mean and what Humphreys means is not exactly the same thing. An immediate difference is that when I argue that computer simulations are concrete computational devices, I do not just mean they are a computer, but rather a computer and all the other concrete architectural, functional and algorithmic elements that allow it to simulate.
} 
ones. ${ }^{27}$ Other times they are not. ${ }^{28}$ While there is a vast literature within philosophy of science concerned with what it means to correctly represent a real system, this will not be the focus of my analysis here. The importance of Humphreys' definition for the purposes of my project is its characterization of system S. For it is system S that is the simulation. ${ }^{29}{ }^{30}$ Furthermore, as I will argue, it is system $\mathrm{S}$ that we should understand as an instrument.

Nevertheless, following my initial definition, and using the terms of Humphreys' definition, we can reframe my original working definition the following way: a computer simulation is a technical artifact, more concretely a computational device — or assemblage of devices ${ }^{31}$ — that produces solutions, via temporal processes (algorithmic procedures that manipulate values and transform symbolic information), to a representational abstraction - that is, a way in which

${ }^{27}$ This should include the simulations processes of simulation processes as well as the simulations of many other computational and robotic processes that would otherwise not deem 'real' systems. Primiero's work (2019) is particularly critical of this limitation on Humphreys' definition. This is an insight that was already acknowledged by early computer scientist as the machines they worked on began to develop the prowess to simulate multiple kinds of solutions to the problems they were solving, i.e. the Monte Carlo method. For more on these early accounts see H. Simon (1969)

${ }^{28}$ It would be interesting, for example, to know, under this definition what to call a computer simulation that failed to correctly represent B or a simulation in which B incorrectly represents R. Under my definition the are simply computer simulations that failed to represent B or computer simulations of an incorrect model respectively.

${ }^{29}$ See footnotes 26,27 , and 28 above.

${ }^{30}$ As I will note in sections below, that this "concrete computational device" is strictly a device whose task is provide solutions to computational (or mathematical) models, or that it in fact does so successfully, is not a completely straightforward characterization of computer simulations. In this section, for example, we will see pretty quickly that this definition only applies to equation-based computer simulations which are derived from mathematical models. Agent-based simulations on the other hand are sometimes produced without a particular model (theoretical, mathematical or computational) in mind.

${ }^{31}$ See footnote 26 above. 
scientist abstract and understand a feature of the world (be it a mathematical model or a set of visual/behavioral inferences) — designed or hypothesized to mimic another system or process.

By a representational abstraction I mean a general, often but not always, informal, way of understanding a phenomenon in the world. At its most basic and broad, a representational abstraction is an epistemic abstraction like any other, a way in which an agent seeking to know a system retrieves and synthesizes relevant ${ }^{32}$ information accessible to him or her to process and understand anything about the world. That is, it can take as many forms as there are of understanding the world: a drawing, a story, a moving picture, or it can be a mathematical equation that mimics the transformations of recognizable values as well as a physical model that retains similarity to salient features of interest. For the purposes of this definition what a representational abstraction is can be left broadly construed. However, because we are dealing with computer simulations, the spectrum of possible abstractions will be limited to what can be implemented on a machine. This, however simple constraint, is yet another way in which the computational device that carries out a computer simulation also narrows what a computer simulation is and can be.

The definition above also captures two other important and desirable aspects of the concept of computer simulation. First, it captures the possibility that a computer simulation can be an

\footnotetext{
${ }^{32}$ Often, particularly upon initial encounters with a phenomenon, the relevant aspects of a system or phenomenon are not in fact known. In these instances what the representational abstraction seeks to depict are the salient elements of an observation: shapes, dimensions, motion patterns, or any perceptible changes thereof.
} 
unsuccessful one. This is because this definition captures computer simulations as an instrument. Technical artifacts such as scientific instruments can be dysfunctional. They are identified in virtue of what they are meant to do even when they fail to do it. For all practical purposes an airplane is still an airplane when it is on the ground, when it fails to lift off, or when it fails to continue in the air. Similarly, for all practical purposes a failed experiment is still an experiment. Computer simulations, understood as technical artifacts, are still so even when they fail to correctly simulate their intended target: they are a failed computer simulation.

Furthermore, the computer simulation is an instrument whose full epistemic status and role within scientific inquiry is still being assessed, as opposed to them being a methodology with sufficient epistemological justification in scientific inquiry as the product of otherwise established practices (as Beisbart, (2017) and Barberousse and Vorms (2014), and others seem to suggest when they posit that we can trust them in virtue of the trust we can allocate to the practices, practitioners, expertise and components that constitute them). That is, my definition of computer simulations captures the fact that whether or not computer simulations are a sound addition to scientific inquiry remains, at least in many instances, something that has yet to be assessed, their ubiquity notwithstanding. This is particularly the case since, as I will argue, we cannot only take into account their predictive powers, or their contributions in areas in which they are the only avenue for inquiry as a model for their sanctioning in all other areas of inquiry.

Secondly, this definition also captures the fact that the processes by which scientists seek to simulate a phenomenon can be hypothetical. That is, the computer simulation, as a technical artifact, can still be understood as a computer simulation even when what it is simulating is only 
hypothesized as being the way the target system is. Consider that we have a case in which the misunderstanding of a natural system is such that none of what we think we know about the system is true. Running a computer simulation with specifications that come from the content of wildly misguided theory can still be understood as a simulation on my view. What the simulation simulates in cases like this is the misguided hypothesized model of a target phenomenon. ${ }^{33}$ This is a highly desirable feature of a definition of computer simulations, because, as it turns out, most simulations are of hypothetical scenarios, some of which are wildly misguided. What I mean by this is the following. At many stages of scientific inquiry, particularly at the stages of documenting observed phenomena, what seem to be the relevant aspects of a system roughly correspond to the immediately salient elements captured by the resources of the scientist. At these stages, the systems characterization is merely hypothesized given the limited information immediately available and does not always correspond to the actually relevant elements of a system. However, we can still build models and/or simulations of misguided, limited or false observations and these simulations can be said to be successfully satisfying the requirements of the term as defined above. ${ }^{34}$

${ }^{33}$ It is generally understood by philosophers of computer simulations that relations of similarity and/or correspondence between a computer simulation and a target phenomenon include complicated and problematic intermediate stages. A computer simulation for example, is often taken to be rather similar to the simulation model (the formal specifications for a computer to run a simulation), the simulation model is in turn taken to be similar to the mathematical model (the formal abstractions taken from a theoretical understanding of a phenomenon), and the mathematical model is seen as having some degree of correspondence to the actual dynamic elements of an observed target system. That this is the case, how it is the case and or whether or not correspondence/similarity relations at one stage entail correspondence at any other stage before it is a big issue in the philosophy of scientific representation. For the most current and thorough examination of these issues see Weisberg (2012), Pincock (2011) and Morrison (2015).

${ }^{34}$ For a thorough investigation of the role of limited and/or false abstractions in scientific inquiry see Pincock, 2011. 


\subsection{Kinds of Computer Simulations}

Computer simulations allow us to trace, track, map and/or predict possible states of a target system. Through iterations and a sequential consolidation of these states they elucidate the temporal, spatial and/or numeric development of a target system. Oftentimes, depending on the character of a specific inquiry this process results in a visual product. When this happens, a simulation is deemed to be the graphic display of a targets' dynamic character. Other times the simulation yields purely numeric outcomes that can be later graphed and compared. Though a visual component can later be tacked on these simulations for presentation purposes, a researcher can 'see' the development of a system through the simulation process by following the numeric results.

In other simulations, the visual aspect of the dynamic character of a system is the starting point of the simulation. That is, as I will explain below, rather than attempting to elicit the behavior of a system from the solutions of a mathematical model, some simulations start from inferences and hypothetical rules that can mimic the visual character of a system in order to gain a more thorough understanding of its mathematical dynamics (Dubucs, 2002). By identifying coarsegrained details in a system such as agents of interest and observed spatial behavior, for example, a computer simulation can be put together without a purely formal (explicitly mathematical) component or a fully integrated theoretical understanding. Examples of this type of simulation are early flock behavior simulations. In order to mimic flock behavior by birds, these simulations were programmed by giving particular agents (birds) on a grid simple update rules. When computed for, these rules prompt complex spatial patterns from a set of agents that resemble 
flock behavior (Reynolds, 1987). By contrast to other simulation techniques, there are no theoretically principled equations at play, no equations to solve.

In what remains of this chapter, section I offer a brief description of the main kinds of computer simulations. I also provide a short description of the role of supercomputers in the implementation of computer simulations. When it comes to the simulation of complex phenomena, supercomputers are often used. The complexity of the facilities in which these computers are housed and maintained as well as the number of experts and components necessary for their use in computer simulations are at the basis of arguments that resist the understanding of computer simulation as a device, a tool or an instrument. Hence, it is also important to provide a brief description of the relationship of supercomputers to contemporary use of computer simulations. This can be found in section 2.2 .3 below. Overall, the following short discussions and descriptions are meant to provide the reader with an understanding of what computer simulations are, what they do, and how they do it. These brief descriptions are also meant to provide a conceptual framework with which to compare and contrast the different kinds of computer simulations to the different interpretative approaches to simulation in the conventional literature. Part of which I have analyzed above and part of which I will analyze in detail in later sections. More importantly, these descriptions aim to reveal to the reader a view of computer simulations as the computational devices they are. 


\subsubsection{Equation based computer simulations}

Equation-based simulations are the product of several transformations of mathematical procedures into something that can be machine implemented and displayed in an interpretable manner. That is, an early motivation to use a computer simulation was to render intractable mathematical problems to somewhat understandable terms (Humphreys, 2004). The process of solving equations often culminates in computational devices also turning the solutions to equations into interpretable visual dynamics such as moving pictures that are meant to represent the distinct and discrete states of a target system (Winsberg, 2019).

Computational systems implement numerical solutions under a critical set of constraints. Strictly speaking, for example, computers do not perform division, but rather they perform a set of addition and subtraction processes that approximates the mathematical function of division. One of the first steps in making a machine that solves an equation is to create specifications for such an equivalent process given constraints of this kind. That is, any multiplication or division is turned into a sequence of addition and subtraction processes that yield equivalent or approximate results. These mathematical equivalences are then translated into code that can specify the instructions of such processes to a machine.

Solutions of this kind are necessarily discretized in that they provide solutions to a given set of inputs iterated within an overarching specified parameter. That is, rather than provide a full solution to a continuous equation, the process provides solutions to segments of the equation at each run and then uses the output of the previous step to calculate the next (Durán, 2018 p.7). So, 
if a computer is trying to solve an equation for the motion of a planet, for example, rather than providing a complete general solution to a linear equation that represents a continuous line, the steps described above would make it so the computer provides a solution to multiple points along a grid that can be interpreted as following a line. Hence, the computer solves equations by providing discretized solutions at iterative steps which depend on the parameters for the length of the solution as well as parameters for the number of iterations necessary. In the case of an equation in this example, which is supposed to be a simulation of a natural phenomenon, the parameters indicating the number of iterations necessary to simulate the phenomenon are provided by contrast with empirical data and not by the original equation or the theoretical principles behind it (Petersen, 2012). If I want to simulate the performance of an airplane, for example, I would make sure my parameters for duration of flight are not unrealistic.

As briefly mentioned above, solving equations via computer simulations of this kind can also just turn the problem into analyzable (intelligible or tractable) solutions that are often displayed in ways that a scientist can read and track (Peterson, 2012; Durán, 2018). The term 'equationbased' is self-explanatory, equation-based simulations are explicitly designed to solve, integrate and follow the discrete solutions to the equations of a theoretically grounded mathematical model (Winsberg, 2019; 2019; Durán, 2018). ${ }^{35}$

\footnotetext{
${ }^{35}$ It is important to note that even some equation-based simulations are not strictly speaking the product of straightforward solutions to differential equations. Some phenomena, such as scramjets, are of such particular complexity that they require fine-grained numerical simulations as well as simulations of disruptive flow patterns within the larger simulations. These simulations are added elements to the conventional Navier-Stokes equations conventionally thought to be sufficient to characterize fluid dynamics (Durán, 2018 p.18).
} 
As we can see from the examples above, however, equation-based simulations (despite their name) are not straightforward equation solvers. Rather they are devices which include a broad and motley set of processes that is capable of yielding results/values that are close enough to those arrived at via an equation. That is - as we will see in more detail in sections 5.2 and 5.2.1 — the processes and procedures that are necessary to render the mathematical or theoretical model onto machine language and then onto interpretable form are filled with practical ad hoc solutions and with considerations regarding engineering constraints that do not follow any of the theoretical principles embedded in the original equations. These processes begin with a wellestablished set of equations that are supposed to mathematically represent — as best as possible given the scientific understanding of the phenomenon of interest - the dynamic features of a target system.

Furthermore, these discretized mathematical solutions are translated into a series of logical steps - a computational model of the mathematical model — that can be coded into a computer language so that a computational device can carry out the simulation. They are specified as instructions to the machine on how best to achieve the operations that can yield the results sought after in the discretized mathematical.

It is important to note that without this last step there is no simulation, which further reaffirms the point made in my definition of computer simulations above: a computer simulation is $a$ concrete computational device - or set of devices - that produces via temporally dynamic 
processes solutions to a representational abstraction designed or hypothesized to mimic another system or process.

A narrow view of computer simulations, defined in detail in the introduction, interprets computer simulations as mere equation solvers, and sees the whole process described above as an extension of the same thing. Namely, mathematical processes. Hence this interpretation neglects to take into account the fact that the approximate discrete values referred to above often take priority over the processes by which they were supposed to arise in the phenomena of interest. While a specific equation dictates a procedure by which to arrive at a given value- - which is, in the case of scientific equations, sometimes derived from the dynamics observed in a phenomenon - its discrete counterpart can be just another completely different way to arrive at the same or at a close-enough value without any regard for theoretical or empirical underpinnings of the original equation. ${ }^{36}$ Consider for a moment some of the examples alluded to above regarding the solving of a linear equation. What is of interest at any given point of the discrete solution is that the position in a grid of a dot is close to where a continuous line would go through. The necessary operations for this approximate and bracketed (a point on a grid) solution are independent from the dynamics of the original equation. That is, if what I need in my grid is for the point in question to be at $(1,3)$, then whatever mathematical operation yields a

\footnotetext{
36 This is a criticism that can be and has been (Parker, 2009; Winsberg, 2010) deployed towards any quantification effort of a natural phenomenon. However, it is important to note that the distinct elements of discretization and coding is often less careful about theoretical underpinnings. There is a sense in which a given differential equation in a conventional mathematical model is the product of a hypothesized mechanism.
} 
value of 3 on the $\mathrm{Y}$ axes and a 1 on the $\mathrm{X}$ axes is good enough. What matters is getting the numerical value and not the procedure by which we attain the value. This is different than a theoretically founded equation in which the dynamics of the variables and their relationships matter and not just the result. Particularly if this equation is supposed to model a real-world target system. Consider a simple application of the original Lotka-Volterra equation in which the aim was to mimic the population dynamics of predator and prey fish in a lake. This equation was based, albeit in an idealized way, ${ }^{37}$ on the actual dynamic between predator and prey species. That is, it is based on the fact that some species eat others and not the other way around. In a discretized model apt for computer analysis all the solutions have to do is approximate the values that the original equation would yield. What the discretized version does not do is take into consideration the real-world dynamics of the phenomena in question. Rather, the focus is on finding whatever set of addition and subtraction processes that would yield the most approximate result.

Computer programmers, at a very basic lower-level programing language, have to consider that for any given value they seek to approximate they have to find a way to arrive at it through binary means, through a set of language-specific functions and write a program that is written to implement these procedures in specific hardware architecture (Resch, 2013; Norton and Suppe, 2001).

\footnotetext{
${ }^{37}$ For a thorough analysis of the function of idealization in scientific representation, and particularly in scientific modelling see Pincock, 2011.
} 
This characterization of equation-based simulations is only a general and straightforward view of what is in principle at play in these simulations. There are several distinct elements that can be part of an equation-based approach. These include techniques that vary from seeking direct and exact solutions to differential equations and mathematical models to the implementation of stochastic techniques when problems are prohibitively complex. ${ }^{38}$ In fact, it may be argued, as some do, that instances of single model straightforward equation-based simulations are no longer the norm. This is in part because the study of complex phenomena often includes the results and the interactions of several subcomponent models.

In any case, what is important to keep in mind is that though these kinds of simulations are strongly based on and derived from mathematical procedures, these procedures do not constitute the simulation itself. Furthermore, the processes by which these mathematical procedures come to be implemented ${ }^{39}$ on a computational device-i.e. the discretization procedures, the coding practices, or the engineering feats - are also not the computer simulation. Rather, the computer simulation ends up being a technical artifact on which the latter are implemented or rather ran.

\footnotetext{
${ }^{38}$ For a thorough review of the specific differences between these kinds of simulations see Durán, (2019) and Winsberg $(2010 ; 2019)$

${ }^{39}$ Directly or indirectly, successfully or unsuccessfully and with the challenges of each of these options - discussed above-notwithstanding.
} 


\subsubsection{Agent-based simulations}

Although the most common interpretations of computer simulation are directly linked to some mathematical procedure, there are other simulations methods that, albeit formal in their implementation, rather follow general rules of behavior and not, strictly speaking, mathematical specifications. Agent based-simulations, for example, do not generally solve the equations of fully developed mathematical models. Rather, these simulations are set to mimic the behavior of a target phenomenon by the implementation of explicit rules by which the various agents defined in the system are supposed to behave. Understanding of a system is therefore sought by 'playing' hypothesized dynamics out and observing the behavioral patterns of a system (Winsberg, 2015; Durán, 2018; Primiero, 2019) and not by seeking solutions to a theoretically-principled equation.

A commonly cited early example of agent-based simulations is the Schelling (1971) model of segregation in which individual agents - dots of one color or another — are placed on a grid and assigned preferential dispositions that would dictate whether they would stay or move in the next iteration. In Schelling's particular simulations the agents were meant to choose their position relative to other agents near them in a simulated neighborhood made of neighbors with similar and dissimilar tendencies and properties. After a few iterations in which these rules played out and the 'agents' - marked squares on a grid—moved, patterns could be discerned and interpreted as a model of segregation based on simple preference rules of affinity and vicinity. That is, based on affinity of marked squares and relative closeness to other marked squares on the grid, the general special organization of these squares would either form clusters of neighboring similar 
squares, disappear, or move towards a general direction in which similar 'neighbors' could be found.

A more neutral description of these kinds of simulations can be found in Symons (2008). Following the work of Hu Richa and Xiaogang's (2003), Symons offers the following description of a general cellular automata model as a quintuple set that involves $\{$ Cells, Cell Space, Cell State, Neighborhoods, Rules\} (Symons, 2008 p. 478), in which:

"[...] cells are the basic objects or elements of the CA each having some individual state depending on the rules of the CA. Cell space is defined as the set of all cells and their values at some time. Neighbors are the set of cells surrounding some any center cell and rules are the transition functions of cell states, mapping cell spaces to cell spaces (Hu Richa and Xiaogang Ru 2003, p. 1047). The rules of the CA are defined as being maximally general with respect to the cells in the model and the application of rules updates each cell synchronically."

Today, many agent-based simulation approaches are hybrid in the sense that while each of the agents active in the simulation are merely responsive through a series of rules, these rules are themselves derived from theoretically-principled equations (Dubucs, 2002).

Although most of the following discussion will directly address computer simulations that are closer to the equation-based type described above, the general point concerning their epistemic standing and their role as the instruments with which the implementation of abstract specifications is made possible applies to both types of computer simulations. In other words, whether we are talking about equation-based simulation or cellular automata simulations, the point remains that the simulation is not the same thing as the specifications it implements, it is not equal to the experimental setting or the theoretical underpinnings from which it is derived, 
designed developed or deployed. Rather, the computer simulation is the means by which the specifications are implemented. In other words, a computer simulation is the thing with which the solutions are carried out, the results are represented, the dynamics are elucidated, etc.

\subsubsection{Supercomputing and Computer Simulations}

At this point, it is important to note a few details about the engineering components (hardware, connectivity, optimization and compatibility efforts) that underlie large scale modern simulation projects of complex phenomena such as weather, exemplified above. This is because, as the phenomena being simulated grows in complexity it is not always possible to consolidate the simulation process in a single computational device. Simulations of large-scale phenomena require the work of multiple computational processes, but also, often, they require the work of multiple full simulations of diverse phenomena. ${ }^{40}$

As noted in the sections above, my definition of computer simulation aims to highlight the computational devices at the core of computer simulations as a key component to understanding them as instruments. ${ }^{41}$ In this section I briefly describe the hardware architecture often required

\footnotetext{
${ }^{40}$ As we will see in detail in section 4.3.1, it is important to note that something being constituted by several devices, procedures or components does not preclude it from being identified and/or understood as a single instrument. This is evident by the mere fact that many things around us - the computer with which I am typing these words, for example - are constituted by a multiplicity of components and can nevertheless be pointed at as a single identifiable device.

${ }^{41}$ In essence, and in virtue of their imperative properties, algorithms and software specification are also artifacts (Turner, 2018) and/or are an integral part of the artifactual nature of computer simulations. This is something I explore elsewhere. In this dissertation the hardware standpoint suffices for my view to work.
} 
to carry out simulations of large and/or complex phenomena or systems. This description is meant to further elucidate the tangible constraints as well as the artifactual nature of computer simulations. As I will show further below, this description also points towards the fact that complex devices, which are constituted by a multiplicity of other devices and require a broad range of experts to carry out a unified function, can also be singled out as single independent artifacts in and of themselves (Simoulin, 2017).

Carrying out simulations of weather prediction systems is often such a complex and large endeavor in scale that multiple computing devices coordinated to execute required operations are required. In large projects, these coordinated devices are grouped together to form computer clusters. In even larger simulation projects — which, given the amounts of data available in today's scientific landscape, are more and more common - these computer clusters are linked to other computer clusters. This is in part because the amount of computational procedures required to render these processes intelligible to researchers in a reasonable time is too large for any one computer. Parallel computational processes allow researchers to compute but also to track, say in a visual manner, the dynamics of a system within a reasonable time and with reasonable efficiency constraints. The interconnected clusters of networked devices that make this possible are called supercomputers.

These supercomputing clusters introduce a layer of additional complexity regarding not only the number and variety of computing components but also the combination of multiple simulations into one set of consolidated outputs. The simulation in such cases is the implementation of all of the elements above in a supercomputing infrastructure. Computer simulations of weather or climate, for example, are the result of 'stitching' together simulations of more specific 
atmospheric phenomena such as cloud formation, air and water current patterns, etc. (Norton and Suppe, 2001; Winsberg, 2010)

Consider the following description of the elements required to follow the detail dynamics of a climate system (Petersen, 2012):

"Comprehensive climate models are based on physical laws represented by mathematical equations that are solved using a three-dimensional grid over the globe. For climate simulation, the major components of the climate system must be represented in sub-models (atmosphere, ocean, land surface, cryosphere and biosphere), along with the processes that go on within and between them. ... Global climate models in which the atmosphere and ocean components have been coupled together are also known as Atmosphere-Ocean General Circulation Models (AOGCMS). In the atmospheric module, for example, equations are solved that describe the large-scale evolution of momentum [of atmospheric 'particles', acp], heat and moisture. Similar equations are solved for the ocean. Currently, the resolution of the atmospheric part of a typical model is about $250 \mathrm{~km}$ in the horizontal and about $1 \mathrm{~km}$ in the vertical above the boundary layer. The resolution of a typical ocean model is about 200 to $400 \mathrm{~m}$ in the vertical, with a horizontal resolution of about 125 to $250 \mathrm{~km}$. Equations are typically solved for every half hour of a model integration [the time-step in the model is half an hour, acp]. Many physical processes, such as those related to clouds or ocean convection, take place on much smaller spatial scales than the model grid and therefore cannot be modelled and resolved explicitly. Their average effects are approximately included in a simple way by taking advantage of physically based relationships with the larger-scale variables. This technique is known as parametrization." (IPCC, 2001; Petersen, 2012)

What is interesting in this quote is that while it mentions all the different types of systems, as well as their associated models and the equations needed to be solved for the models to yield useful results for each of the levels of the simulation - from ocean currents to cloud dynamicslittle is said about how each of the results is incorporated into the whole to provide a “comprehensive climate model." However, it is here that much of interest happens besides the calculation of numerical results, or the solving of equations. It is at the incorporating stage that much extra-mathematical and extra-theoretical work (Winsberg, 2010; 2019) is needed. As is 
evidenced in this description, although equations are at the core of the simulation processes, the results of each computation still have to be coupled together and/or extrapolated for use at lower or higher levels of the modeling process.

These instruments sometimes rely on approximate, discretized behavior of mathematical models of the natural systems of interest. In simulations of dynamic process that are mathematically driven, the algorithm coded into the software itself is intended to implement a function that takes as an input a representation of some initial state of the natural system and gives as an output the state of the system at some later time. Alternatively, other kinds of computer simulation seek to mimic the spatial development or changes of discrete states of a phenomenon's behavior. ${ }^{42}$ When a system of agents is discretized in a way in which it can be represented as a position on a grid with specified rules that dictate its future states, then a dynamic representation of a behavior can be graphically simulated. In these types of simulation, rules-hypothetical or theoretical—are coded onto software procedures to visually represent a desired display. These rules along with subsequent iterations of future states may elicit a similar computational representation to the observed behavior of a target system. ${ }^{43}$ The electronic devices carrying out both of the processes

\footnotetext{
${ }^{42}$ As mentioned above, simulations known as Cellular Automata, for example, are often used to track the iterated spatial relationships of agents represented on a grid. This type of simulation often differs from those that solve equations in that the dynamic representation of the agents on the grid is often guided by behavioral rules rather than solutions to equations and/or rather than through strictly formal theoretical principles.

${ }^{43}$ A key distinction here is that not all simulations are mathematically, or theoretically principled (i.e. with sound and/or proven scientific explanations and/or with axiomatic understanding). Oftentimes, the understanding of a target system is so that only visual cues and clues are available to researchers. Consider that before we understood the dynamics of bird flock formation in birds the only available information was the formations themselves and not any strong theoretical framework and/or mathematical foundation that informed our understanding of the phenomenon. Nevertheless, this visual information was sometimes sufficient to hypothesize possible ways things
} 
briefly described above often repeat this operation on the outputs of previous iterations of the operation and create a sequence of representations that can shed light on the unfolding dynamics of the natural system.

As the citation above shows, and as I will discuss in detail below, the implementation of computer simulations is comprised of several subsequent steps that integrate components and procedures of many different kinds and at many different levels. This process of integration is often described as a simulation pipeline (Winsberg, 2010; Resch, 2013; 2017) in which researchers start by identifying a phenomenon to be simulated and then gradually move from the formalization of a model (mathematical/computational/logical) of such phenomenon to its implementation on a computing architecture capable of distributing the work in an appropriate manner. In practice, this process includes an arduous collaboration process between scientists, engineers, and programmers. This is particularly the case when the phenomenon in question is something as complex as the variation of wind and sea currents in large portions of an ocean (Kaminski, 2017). Not only is a mathematical model not sufficient by itself to establish a simulation process, but none of the elements remain untouched by the conversation between experts. Engineers handling the computing machinery have to adapt the architecture for the distribution of the necessary computing processes associated with the model. Programmers have

worked, sometimes it was enough to even hypothesize a formal dynamic which did not have a strong theoretical foundation regarding anything about birds (Heppner and Grenander, 1990). In these cases, makeshift code enables a software system to mimic expected or desired visual behavior. Other time formal rules are used to mimic such behavior despite the fact that they knowingly do not correspond to the underlying mechanisms by which the target system develops. 
to ensure the code is written in a way and in a language that is compatible with such architecture, but furthermore even after this is done, the code still has to be "optimized" for efficiency and other considerations. Often, in this process the numerical elements of the model that discretize the original equations associated with the phenomenon to be simulated also have to be adapted to ensure that the approximate results are still achievable under the logical configuration of the architectural distribution of computing power.

Furthermore, the description in the citation above also signals that in simulations of this scale each of the computation processes is the result of a completely different model which in turn signals the possibility of there being different assumptions built into each step. There are also distinct machines operating at distinct levels of computation. A super computer may not be needed for smaller scale dynamics, but it is definitely needed to consolidate the distinct models and results into a single simulation.

However, as we saw above in the discussion of equation-based simulations, solving numeric problems, that is providing approximate discrete values that correspond to continuous equations, is but one function of the simulation. ${ }^{44}$ Rather simulations are in fact comprised of an amalgam

\footnotetext{
${ }^{44}$ It is important to note here that while, conventionally, the differences between a continuous and a discrete computational process have been equated to the differences between analog and digital computation, the comparison does not hold. Hence, when I refer to discrete rather than continuous mathematical processes, I am not speaking about the difference between analog and digital computer simulations but rather about the mathematics used for the solutions in the models they implement. For a thorough distinction between analog, continuous, digital and discrete computation see Maley (2011). The claim here is merely that there are mathematical methods that yield solutions to mathematical problems in a continuous manner and there are methods that provide discretized solutions. It is often the case that when it comes to the implementation of computer simulations the former must be translated to the latter to accommodate existing computational capabilities, languages, and resources. This, in and of itself, represents a
} 
of functions, methods, practices and constraints, which often include practical extra-theoretical and ad hoc engineering and programming techniques to overcome these constraints (Winsberg, 2010 p.46). They include sometimes very informal "assumptions about what parameters to include or neglect, rules of thumb about how to overcome computational difficulties- what model assumptions to use, what differencing scheme to employ, what symmetries to exploit- graphical techniques for visualizing data, and techniques for comparing and calibrating simulations results to known experimental and observational data." (Winsberg, 2010, p.45) These practices are integrating efforts that fall well beyond both the scientific theory and the mathematical model of the phenomenon they are trying to simulate. In other words, as expressed in earlier sections and as will be discussed in detail in sections below, these practices incorporate extra-mathematical and extra-theoretical elements (Winsberg, 2019). This fact, that elements beyond the formal aspects of the mathematical models or the theoretical framework have to be considered, is important because it introduces two things. First, it introduces a conceptual distance between the formal elements of scientific inquiry, such as mathematical procedures and theoretical models, and their implementation in a computational device. Hence, we can already conceive at least a conceptual independence ${ }^{45}$ between these elements as constituents of and not identical to computer simulations. Secondly, these considerations of extra theoretical/mathematical elements

non-trivial alteration of the mathematical, procedural and theoretical content of conventional scientific models turned computer simulations.

${ }^{45}$ Here I am using the word independence not as an absolute term. It may still be the case, as I will later argue, that sound scientific use of computer simulations in fact necessitates strong theoretical and empirical anchoring. However, the independence here is meant to elucidate the conceptual distinction between one and the other thing. That is, neither the mathematical procedures nor the theoretical models are the computer simulation. 
bring forth the role of hardware architecture and engineering efforts behind a computer simulation. In other words, it highlights once more, its instrument-like features.

Consider for a moment the Large Hadron Collider. While it is one of the most complex scientific instruments ever created, requiring a large heterogeneous community of experts and a monumental number of components, it would not be appropriate to consider the large hadron collider a 'practice', or a model. It is an instrument with which experimental practices can be carried out and models tested. But it is also the case that some of these large complex structures can become different kinds of instruments and perform different functions at different times. It is what Simoulin calls a polymorph instrument (2017 p.425) in that it can be adapted and changed for many specific uses. Computers as general-purpose machines, and supercomputers by extension are also polymorph instruments. Supercomputers, which are made of computer clusters are polymorph instruments constituted by polymorph instruments. As I will argue in later sections of this dissertation, computer simulations are too.

In short, there are a multiplicity of heterogenous processes and components in a computer simulation. Nevertheless, as briefly explained above and will become clear in future sections of this dissertation, that this is the case does not exclude these simulations from being treated as a single product (Simoulin, 2017), an artifact whose reliability and epistemic role cannot be assessed in the same way we asses other instruments. Rather, as instruments, they integrate many straightforward and single model simulations and produce a somewhat intelligible presentation of the aggregated product. Simulation and prediction methods such as the one exemplified by the quote above, for example, which produce simulations of both regional weather patterns and 
global trends in large systems, incorporate daily terabytes of data produced and processed by ocean, land and ice surface models of earth. They also take into consideration parameterized information from cloud formation models, temperature models as well as past and future climate simulations (Kauffmann and Smarr, 1993; Boschetti and Symons 2011). Hence, as mentioned above, these simulations are often composed of independent simulations of phenomena at multiple levels and scales. As we will see below, that large scale complex computer simulations are constituted by multiple and diverse components, processes, and put together through the efforts of similarly motley expert communities will serve as the basis for the arguments of those that think that the term 'instrument' is inadequate to capture the nature of computer simulations. I will show this not to be the case. 


\section{Computer Simulations as Instruments}

In this chapter I present a positive account in support of the view of computer simulations as instruments. As I will show, there are several preliminary steps in order to achieve a solid framework for this argument to work: starting by establishing that, whatever they are, computer simulations are at the very least something separate and distinct from their theoretical and practical aspects. Secondly, I will also show that at their very core, computer simulations are technical artifacts and that their distinctness can be functionally identified as separate from the formal methods and the experimental practices for and in which they are deployed. After that is established, I will also show that the reason why philosophers have a difficult time finding a category with which to characterize computer simulations within scientific inquiry is because they are in fact hybrid instruments. This explains their often-cited in-betweenness that many (Lenhard, 2007; Morgan and Morrison, 1999) have tried to characterize as being in between theory and experiment. Here I suggest that a better explanation is that they are indeed in between theory and experiment, but not because they are a third branch of inquiry, or because they are special kind of experiment. Rather, they are in between theory and experiments because they are instruments and all scientific instrument share this feature. It is the category of instruments that is in between experiments and theory and it is instruments that represent a third branch of inquiry (Baird, 2004). However, as I will make clear, computer simulations are also in between when it comes to the taxonomy of functions and roles of other instruments in the philosophical and historical literature of scientific inquiry. 
My main claim in this chapter is that computer simulations are instruments. Hence, they can and should be vetted, tested and sanctioned with the same normative scrutiny that other instruments are. $^{46}$

\subsection{Computer Simulation as Distinct}

A theoretical or abstract model of the kind conventionally used in science is a conceptual construct that stipulates the relationships and/or the dynamic transformations of a system and the relationships of the entities therein (Pincock, 2011). These models abstract and describe the scientifically salient features of a system. As such, they offer an abstract and often formal representation of a target system (Durán, 2018). In contrast, as we will see later in this section, computer simulations (of dynamic target systems) are technological artifacts - physical implementations of specifications ${ }^{47}$ — that implement/execute the computational processes required to follow the progression of these specifications and descriptions. While they may be the product of, or contain within them, the specifications of a conceptual model, computer simulations are something other than the model itself: they are often the implementation (through

\footnotetext{
${ }^{46}$ This in a sense constitutes a secondary call for models and modeling practices to also be undertaken with the same normative stringency as other instruments and instrument-making practices before they are allowed widespread application in scientific inquiry. As I will argue at the end of this dissertation, selective curve fitting endeavors as replacements for truthfulness (Hubig and Kaminski, 2017) as well as assessments of predictive prowess alone do not satisfy fundamental normative requirements of scientific practices that call for desiderata such as transparency, understanding, replicability, etc.

${ }^{47}$ It is important to note that digital implementations also fit this description.
} 
hardware architecture and software specifications) of said models. But sometimes they are something else, something more. Hence at the most basic level, a distinction can be drawn between the model and a computer simulation of that model by noting that the model is not the simulation and vice versa. They are two distinct things. This point applies even when the computer simulation is simply characterized as the implementation of a model.

Though I will argue in detail that computer simulations are distinct from formal abstract artifacts such as models and equations in terms of the non-identity of properties and/or functions between them, for now we can also just see this intuitively by considering the fact that computer simulation would not be needed if they did not do something other than what the model already does, or if computer simulations did not do it differently, i.e. faster, automatically, etc. Though this is not a conclusive argument in favor of distinctness, it is an intuitive way of getting at the fact that computer simulations were originally devised as mechanical ways to facilitate an otherwise time-consuming — and sometimes intractable — problem (Fox-Keller, 2003). If formal models, equations, and or simple understanding of theoretical principles and their dynamics were sufficient — and/or manageable — to provide what was required for the inquiry in question, computer simulations would have been simply continued developing as yet another kind of calculator, albeit more complicated. As we saw in chapter 2 and will discuss in detail again in section 4.2 , this was not the case. Rather, computer simulations were quickly applied to tasks beyond their early numerical tasks to encompass a wide range of procedures beyond calculation, i.e. the sorting of dynamic development of a represented system, conversion of information of one kind onto another, visualization, etc. That is, computer simulations, soon after their initial development, were deployed in contexts in which their function was to track, trace and represent, 
either through a print-out or a screen, the spatial changes of system and its components. These processes often included the transformation of numerical values, solved for by the computational process, to be turned into graphic spatial information and therefore into visual data. In this sense, yet again, we can see that the entirety of a computer simulation and its functions were often something else than arithmetic solvers when deployed in inquiry. Furthermore, their results were only such once the entirety of the formal specifications were iteratively implemented. That is, not only did the specifications had to be run on machines, but for something to be called a simulation, they had to be run multiple times. In fact, many times. Without these extra elements - the graphic displays, the visual dynamics, the iterations - the solutions to complicated mathematical models would yield little to no information to a researcher. ${ }^{48}$ Yielding numerical values for the many particles in a gaseous system, or tracking the complex dynamic features of cloud formation purely with numbers is not an efficient epistemic strategy for human understanding.

Furthermore, as already discussed in section 2.3 , there are many ways in which computer simulations, the complex technological artifacts with which dynamic systems are studied, are not identical to any of the preliminary stages required for their development. A computer simulation, for example, is either the sum of the complete set of stages represented in the simulation pipeline (specified in detail in section 4.1), or is only one of the stages - a stage between the actual implementation of functional specifications and its results: a product of the process. It can be

\footnotetext{
${ }^{48}$ Spreadsheets and lists of calculations provided important insights but here again we see that the spreadsheet and its structural features was an essential component in the intelligibility of results and their implications.
} 
easily inferred, for example, that computer simulations are in fact neither the complete set of stages preliminary to their yielding of results, nor are they their results. Rather, they are a final product of the preliminary stages, namely a machine with which results can be acquired. Either way, the point here is that however many different ways one choses to flesh out the process of creating them, computer simulations are a distinct thing from those processes in and of themselves.

For the sake of clarity, let us review some of the ways in which computer simulations are distinct from the constituent aspects of their construction (Winsberg, 2010; Resch et al., 2017). These constituent aspects, the programming, the multidisciplinary social dynamics, the hardware architecture $^{49}$, etc., are often discussed in the contemporary literature of computer simulations as if they were the same things as a computer simulation. Here, I argue that this is not the case.

First, as I have discussed in previous sections, the mathematical operations that form the basis of computer simulations of dynamic systems, for example, are quite different from those found in the theoretical stages of inquiry. If, for example, we are talking about a computer simulation that springs from inquiry with largely established theoretical principles and the mathematical equations associated with them, we can quickly see that these latter equations are seldom, if ever, directly part of the computer simulation itself. Consider, for example, that the discrete

\footnotetext{
${ }^{49}$ It is important to note that for a simulation to be a simulation under my definition (in section 2.1.), it requires all the elements to be considered. While it is true that I rely strongly on the physical aspect of their implementation for the instruments view of computer simulations, there is an equal weight given to the implementation aspects which includes things like the 'running' of formal specification, iterations, etc.
} 
translations of the equations required to compute the dynamic stages of a represented system do not yield the exact same results generated by their continuous counterparts. As I have noted several times before, the way a computer simulation solves an equation is by providing approximate values to discretized parameters that roughly correspond to the results one gets from a continuous equation. While the results can be similar and/or approximate to an almost negligible degree, the fact remains that both the results and the methods are distinct. Often this difference is not only a matter of transformation but of finding completely different and independent ways of achieving these similar-enough results. This point already signals, at the very least, a departure - a gap — between the originating formal aspects of inquiry and their machine-implemented counterpart in computer simulations. Second, the programming code by which the discrete operations are specified for the machines to implement are not the same as the mathematical operations themselves. Whether they are continuous or discrete, the machine must understand what to do in order to carry out those operations and this is specified through a set of logical commands that often deviate from the principles of mathematics that guide either the discrete or the continuous operations (Winsberg, 2010). In fact, the procedures by which the machines execute these logical commands are often the result engineering ingenuity that has little to no formal basis. These two stages of the design, development and deployment of computer simulations alone already constitutes grounds for distinction between computer simulations and mathematical models, whether these models are computer based or not, but also between the coding and the machine execution of such code. At this point it should be rather obvious that computer simulations are not the mathematical models they implement, the equations in such models, or any of the formal aspects that constitute them, which are in many ways are intangible, abstract constructs (Durán, 2018 p.33). 
So, by now it should be clear that computer simulations are not ${ }^{50}$ the abstract components typically associated with the theoretical aspects of their constructions. They are not the mathematical processes they implement, or the formal specifications from whence they are built, or the programs they run, or the symbolic content they manipulate. They are simply distinct from these.

Very similar points can be made about the relationship between computer simulations and experimental settings. A computer simulation can be the software/hardware implementation of experimental specifications (Barberousse, 2019). Barberousse (2019), for example argues that full experimental settings that include procedures, computations, controls and data manipulations can be encoded in the programs we use in simulations as well as in the architecture used to run them. That is, full specifications and descriptions of an experimental procedure can be encoded for a machine to execute - or more accurately, as we will see below, to simulate. But it is this functionality, the capacity and/or inherent design to simulate that precisely separates computer simulations from what an experiment is, from what an experimental setting comprises, and from what an experiment's description and specifications are and do. As will be made clear in the following section, a description of an experiment does not simulate the experiment. Specifications are not simulations. And just like a microscope is not strictly speaking the

${ }^{50}$ In a more formal sense, it can be said that computer simulations are simply not identical (in the $\mathrm{A}=\mathrm{A}$ sense) to their constituent components in any way they may be fleshed out. Even if computer simulations are understood to be the product of all of these constituent components, as a whole, it is undeniable that their conceptual identification and their practical deployment, as I will argue below, is enough to distinguish them from them. 
experiment but rather an instrument with which experiments can be carried out, a computer simulation is also not an experiment but the thing with which an experiment can be simulated. ${ }^{51}$

In other words, computer simulations are simply distinct from the processes and components that constitute them. However, to be clear, this is not a deep emergentist claim about the nature and properties of computer simulations. ${ }^{52}$ Rather, it is a claim about the relatively straightforward independence of an object, in this case a technical artifact, vis-à-vis its constituents. Just like a car is distinct from its engine, its tires and its dashboard though it cannot be without them, a computer simulation is distinct from its underlying constituents. It can be conceptually identified as a separate object. ${ }^{53}$ That is, the car, as a whole, is an object that can be thought of, discussed, assessed, entertained without explicit reference to any particular component. So can a computer simulation. Furthermore, as I will discuss in detail in the following section, this distinction between a computer simulation and its components can be further characterized in terms of their respective functions. A computer simulation does things and is deployed to do things that none

\footnotetext{
${ }^{51}$ It must be noted that a computer simulation can be experimented on and/or can be an experiment itself, that is the subject of inquiry itself. This is also true of telescopes and other instruments of inquiry. In fact, historically speaking, it is the way in which instruments develop and how some of them become part of the precision industry that ensures the robust reliability required of them to be deployed in scientific settings. This does not mean, however, that computer simulations, when deployed to simulate an experimental setting or when deployed within an experimental setting, are the experiment themselves.

${ }^{52}$ Of course, the case can always be made that some of the properties of computer simulations and some of the behaviors of some simulated systems do in fact elicit emergent features. However, this is not the claim I am making and delving into such cases would be a significant distraction from what I intend to show in this section.

${ }^{53}$ Of course, a car is not independent from its constituents in a strict sense of the word, but things can be said about it that do not necessarily apply to any of its components or that apply to many rather as a whole.
} 
of its constituent components do. When they are deployed to do similar things, they do them differently. If this were not the case, it would be difficult to justify their use.

\subsubsection{Computer simulations as functionally distinct}

In the paragraphs above I showed that at a very basic level, intuitively, computer simulations are simply distinct from their constitutive formal aspects and/or the experimental practices for which they are deployed. Whatever they are, my definition in chapter 2 notwithstanding, they are not the models and they are not the experiments with which they are often equated, or subsumed under, in the philosophical literature. Here I will show that computer simulations are also distinct from conceptual models or experiments in that, oftentimes, they simply carry out different tasks than those of models and/or experimental practices. They do not only compute but also automate computations, they do not only represent but also transform information from one kind to another (numeric/visual), etc. When the functions of computer simulations do overlap with the functions that can be carried out by other methods then they are carried out in a different way. That is, they are automatic, faster, more accurate, approximate and/or simply more convenient. ${ }^{54}$ Early successful development of computer simulations was deployed predominantly in order to "bypass the mathematical intractability of equations conventionally used to describe" highly non-linear phenomena (Fox-Keller, 2003). This is evidence that these technological artifacts

\footnotetext{
${ }^{54} \mathrm{We}$ can think of a computer simulation that takes as long as a human to carry out the necessary computations of a represented system and yet it would still be convenient for the human to not carry them herself while pursuing other aspects of the inquiry in question.
} 
were conceived of, designed with, developed for and deployed in the service of a task other than and/or different than the tasks that could be carried by any other of the elements of inquiry at the inquirer's disposal at the time, including the very methods they were developed to mimic.

Again, at a fundamental level, the task of the computer simulation to simulate, as briefly mentioned above, is something that is not done by either the formal elements, such as a theoretical model, or any experimental practice encoded onto the set of artifacts that comprise it. This is why a computer simulation is not a calculator (Fox-Keller, 2003) or strictly a formal model (Winsberg, 2010), or even an experiment in the conventional ${ }^{55}$ sense (Morrison, 2015): it does something else.

More importantly, a computer simulation is designed to do something else. It is this intentional design that is the core of what makes artifacts distinct from one another and from other things, e.g. organisms (Symons, 2010) and/or other natural objects with functional propertiespseudoartifacts (Kroes, 2006). The functions for which they are designed are particularly

\footnotetext{
${ }_{55}$ Morrison (2015), Barberousse and Vorms, (2014) and others that defend the capacity of computer simulations to furnish empirical, a posteriori, knowledge have had to grapple with the fact that computer simulations do not interact with a phenomenon the way most experimental practice is thought to do: direct material manipulation, causal interventions, etc., are not what simulations do. In order to make sense of the empirical content extracted by the use of computer simulations a few notable and questionable strategies have emerged. As I will show in this section, Morrison relies on a deflationary view of some established experimental practices and deems them to be nothing more than the manipulation of theoretical values. If computer simulations can be said to do the same then they are in equal epistemic footing, i.e. we could not say that computer simulations do not provide empirical insight without saying that these other established experimental practices fail to do so as well. Similarly, Barberousse and Vorms, corner their readers into an argumentative conundrum that relies on considering computer simulations as reliable transmitters of knowledge as testimony and memory. They also argue that the labels of 'a priori' or 'posteriori' do not apply to knowledge per se but rather to the warrants on which knowledge is based. Denying that computer simulations can transmit knowledge (with its respective warrants) would also deny that many forms of established transmission (expert testimony, textbooks, etc.) would fail to do so too.
} 
important to technical artifacts and more so when these technical artifacts are deployed in scientific contexts where, as I will show in the last section of this dissertation, epistemic requirements are stricter than those in ordinary epistemic experience. A computer simulation, as we will see in the following sections, is a technical artifact—a physical construct (Kroes, 2003) with a specified function at the design level (Symons, 2010). While a computer simulation can be used for many broadly construed scientific purposes — explanation, experimentation, etc.that may overlap with other elements of inquiry, like models or experiments, they are, at their core, designed to simulate. This function is different from that of the model, or the experimental practice. In a simple way, it is a technological artifact designed to run the model(s) or the comparative processes specified by the experimental procedures encoded in it. It is designed to represent - albeit indirectly — in a performative manner the dynamic progressions of a system specified in a model or an experimental setting.

Let us consider the following objection to my view of the functional distinctiveness of computer simulations. A conceptual model, it may be said, is also an artifact that is designed with the specific task to represent the dynamic processes of a target system. It does so through a set of either theoretical or empirical directives meant to help the user of the model or the experimenter replicate, in one way or another ${ }^{56}$, the dynamic features of a target process. In this sense, both the model and the simulation have the same function, namely to represent a target phenomenon. If this is so, their functionality is not a basis for their differentiation. This objection- which can

\footnotetext{
${ }^{56}$ Mathematically, imaginatively, intuitively.
} 
easily be a product of those views that take computer simulations to be mere extensions of mathematical models, logical abstractions and/or other formal methodology in inquiry (Weisberg, 2012; Pincock, 2011) as well as from those views that acknowledge that computer simulations can encapsulate experimental settings (Barberousse, 2019) — is an objection to my view only if one neglects the fact that computer simulations are a sort of hybrid instrument (Baird, 2004 p. 5) which, much like a measuring instrument ${ }^{57}$, must perform to represent.

That is, in order for the computer simulation to do the representing part it must carry out the processes by which it represents. Without the execution of procedures, a computer simulation is not a computer simulation but merely a description or conceptual specification of said procedures. In order for the computer simulation to be a computer simulation (particularly of a dynamic target) it must run. This is similar to the hybridity of instruments such as the thermometer in which a process, a transformation of its components, must occur in order for the thermometer to display its representative function (Baird, 2004). A conceptual model by contrast does not have to perform to represent, it does not have to execute the specifications it contains. A model can be specified by a static representation in which the necessary connections and transformation are simply described. This can be done in many different ways: a drawing, a textual description, or even an equation, amongst others. Yet, neither the drawing nor the textual

\footnotetext{
${ }^{57}$ As we will see this comparison will be very important when I discuss Margaret Morrison's (2015) defense of the equal epistemic status between computer simulations and instruments by way of comparing computer simulations to precisely measuring instruments. As I will show, however, Morrison's strategy to equate the epistemic status of computer simulations to that of empirical experiments is by holding a deflationary view of the latter by pointing towards the abstract aspect of value manipulation in many experimental practices in order to position the latter at the same disadvantage of the former and not the other way around. This, in turn, leaves the discussion of the materiality of computer simulations aside and focuses more in the abstract aspect of their manipulation of theoretical values.
} 
description carries out—performs — the specified operations, they simply describe them.

Similarly, an equation can be used to carry out the operations but it does not perform them itself.

They are static, descriptive artifacts removed — much like a conceptual model—from things like

motion and change (Baird, 2004; Durán, 2018). In contrast, a computer simulation is a

technological artifact that has as its main function to simulate, mimic, through the prescribed

specifications the dynamic transformations of the simulated target. This performative aspect is

the physical implementation of the functional specifications, which often if not always, require

iterations of the formal specifications. The functions of a computer simulation specified that

something must be done, operations must be executed on its contents and not merely described. ${ }^{58}$

Consider that a computer simulation is not the end result of dynamic calculations, nor is it the

final state of the processes of the target system being simulated. Rather, a computer simulation is

the execution of the processes meant to represent the dynamic development of the target

phenomenon.

To have a clearer view of this last point consider the following: if I was travelling in my

intergalactic ship in space and I asked my computer assistant to provide me with a still image of

a specific point in time in future galactic formations, this image would not be a simulation. This

\footnotetext{
${ }^{58}$ It is important to note that this performative aspect of the dynamic features of a computer simulation is not reducible to mere perceived movement. That is, a computer simulation is also not just the moving pictures often accompanying the transmission of its results. This visual result of a captured computer simulation is yet another thing, separate from the simulation itself. The visual representation of the simulation is only one of the ways in which the simulation can be captured for future analysis. The simulation is the carrying out of the mimicry inherent in the concept; the simulation is what is while it is being done. When the computer simulation simulates, it does so in the present tense. This function of simulating is carried out by the physical implementation of its functional specifications.
} 
is the case even if the image was produced by following formal specifications, models, equations, etc. It is still just an image and not a simulation. The image may even be the product of a simulation, but it is not the simulation itself. Furthermore, consider that I ask my computer assistant to produce merely a numeric representation of the position of a specific star within this galactic formation in the future: this result is also not the simulation, though it may have been acquired through it. The simulation happens somewhere after the specifications are implemented and before the results are produced.

Besides implementing the specifications of conceptual models and other formal specifications, a computer simulation can also be designed to compute and represent the progression of the transformations of values in an experimental setting. And just as with the example above regarding the extra functional aspect of computer simulations in relation to conceptual models, the distinction is evident here once more: computer simulations can and do simulate an experimental setting (Barberousse, 2019). Once again, in this sense, a computer simulation has a different function from that of the model or that of the experiment: it is an artifact designed to simulate them. A model of a system can, and is often, constructed with the dynamic provisos of a target system so that it can, when implemented on a separate artifact, provide the necessary specifications for this separate artifact— the simulation — to mimic the behavior (dynamic development) of said system. Yet the model does not itself constitute a simulation. The model does not run the model, the experiment does not run the experiment, nor do they simulate themselves - that is, they do not mimic/represent themselves: the computer simulation does. 
Similarly, an experimental setting can contain the necessary specifications for the transformations of values required for a specific inquiry and a computer simulation can be thought of as an artifact devised to automate these processes (Lenhard, 2007). And yet even here there is a further important distinction that must be drawn. The way computer simulations can automate some aspects of some experimental practices ${ }^{59}$ is by simulating the equivalent values (initial conditions, input data, expected parameters, etc.) of a specified system and their respective numerical transformations through roughly equivalent discrete methods in coded circuitry. The computer simulation does not run the experiment in a conventional understanding of experimental practice. That is, while the same specifications could be given to a team of researchers for them to run an actual experiment in which direct observations and manipulations are conducted in order to extract data from a target phenomenon in the world, the computer simulation can only simulate this process: hence the name. Saying that a computer simulation runs an experiment means that a computer simulation simulates — mimics, if you will— the experiment being carried out by executing the value transformations specified by the experimental stipulations. ${ }^{60}$ In this way, then, the automation that takes place in the computer simulation of the experiment is that of the simulated processes by which the values and data of the experimental setting are transformed and unfolded. In this sense, computer simulations

\footnotetext{
${ }^{59}$ As we will see below Margaret Morrison narrows the kind of experimental practices in question to those in which the 'experiment' consists on comparing and manipulating theoretical values and observing not the direct transformations of the phenomenon in question but rather the transformations of the numerical values entered and contrasted through the appropriate modeling techniques and from appropriate data structures.

${ }^{60}$ This will be an important point going forward as I analyze, in paragraphs below, the deflationary accounts of both experiments and computer simulations that Margaret Morrison endorses in order to equate the epistemic role that both inquiry methods play in scientific inquiry.
} 
encapsulate (Baird, 2004) the required steps for the transformations of values specified by the inquiry and by carrying out these steps they simulate the steps that would have been taken on a laboratory setting. The computational architecture at the heart of these technological artifacts processes these steps and further transforms the outputs (inferences, calculations) onto intelligible results.

In short, whatever it is they do, what computer simulations do is not done, or is not done in the same way, by theoretical elements, or models. Similarly, whatever they do, is also not what the experiment does. Rather they are the thing with which the experimental values are entertained, the thing which the entities and transformations of a target in the real world are mimicked, or the thing with which the experimental procedures are automated, etc. Once again, the key here is the words "thing with which". Conceptual elements of inquiry such as theories, their models, equations and other formal components are abstractions (Weisberg, 2012; Pincock, 2011). As such they are composed of propositions, claims, beliefs, etc. They are made of the kinds of things that are not subject to physical forces (Durán, 2018). Instruments, on the other hand, are specialized physical constructions. If, as Baird (2004) suggests, instruments "whatever they may be, are not beliefs" (p.4), neither are computer simulations. The point is that even reductionists views - views that attempt to reduce computer simulations to either theoretical elements or empirical elements of inquiry - cannot deny the individuality of the artifact in question: the computer simulation. If only in virtue of the fact that it is doing something else than any of the things in which it depends (its constituents). This is, as Baird (2004) would agree, evidence of the individuality of artifacts in general (Symons, 2010). That is, computer simulations can be individuated by the fact that they perform a different function than that of models, they carry an 
extra/independent task from those of their constituents: computer simulations are the things with which we can process models, solve equations, transform/translate, display, model, etc.

\subsection{The In-Betweenness of Computer Simulations}

The discussion above has as its aim to establish, through a set of distinctions, the ontological independence of computer simulations from other elements of inquiry such as conceptual models - often associated more closely to theoretical endeavors - and experimental practice. This ontological independence does not entail that theory or experimental practice do not have an influence on how computer simulations are designed, developed and/or deployed. Rather, it shows that, at the very least, despite their inherent relationships with these other elements of inquiry, computer simulations are a separate artifact with which inquiry can be conducted.

This much has been recognized from the early onset of philosophical debates that sought to understand the nature and import of this seemingly novel addition to scientific inquiry. While some have simply denied that computer simulations represent an unprecedented challenge in any relevant debate in the philosophy of science (See Frigg and Reiss, 2009), many (Humphreys, 2004; 2009; Winsberg, 2010; Fox-Keller, 2003; Morrison, 2015; Resch et al., 2017) have argued for the distinct character of computer simulations by — explicitly or implicitly — choosing a side in the dichotomy described above between theoretical endeavors and experimental practice and then showing how computer simulations fit in to one of those categories and/or differs from other members of those two categories. Some philosophers, for example, think that computer simulations are special cases of experimental procedures; others suggest that computer simulations are special cases of conceptual models (Weisberg, 2012; Pincock, 2011). As I 
showed in the section above, and as others have detailed, computer simulations are not easily boxed in the categories conventionally discussed in the literature of epistemology of science: they do not easily fit in the theoretical toolbox, nor do they fit in the empirical toolbox. When the discrepancies have been too salient to accommodate, an argumentative strategy has been to accept that computer simulations must be in fact a strange and novel element in scientific inquiry. That is, they are seen as a completely new way of acquiring knowledge in between theoretical aspects of inquiry and experimental methods (Lenhard, 2007; Resch et al, 2017). By positing that computer simulations are neither conventional members of the set of theoretical endeavors in the books or members of the set of established experimental practices philosophers such as Kaminski and others have begun to make sense of their epistemic role in scientific inquiry by suggesting that, as completely novel additions to science, computer simulations are epistemologically speaking somewhere in between experiment and theory.

These views are somewhat correct. Computer simulations do show a dual nature of sorts, and they do not belong on either side of such a dichotomy. However, here I argue that computer simulations are not epistemically in-between because of their novel methodological characteristics — as Lenhard (2007) suggests. I also argue that they are not in between solely because of the fact that they can function as other measurement practices do, a suggestion made by Margaret Morrison as a means to accommodate the seemingly ambiguous epistemic nature of computer simulations. Rather, I argue, the reason why computer simulations are somewhere in between experiment and theory is because they are in fact neither. That is, computer simulations do not belong to either the set of theoretical undertakings nor to the set of experimental practices. Just like a stethoscope which is neither a purely theoretical construct in medicine nor an 
experimental practice in and of itself, computer simulations are also neither. Stethoscopes can be used properly when they are theoretically informed. That is, when their design, development and deployment emerge from a deeper conceptual understanding of the phenomenon and its relation to inquiry. Stethoscopes can also be an adequate device in an experimental setting within medicine. Yet, they are neither the theory, the experiment or medicine itself. Rather, they are instruments designed, developed and deployed in clinical practices of medicine. Similarly, computer simulations belong to a third — and equally important (Heilbron, 1993; Van Helden, 1993; Baird, 2004 p.89) —element of scientific inquiry: namely, instruments. As such, they can elicit an epistemic independence, they can serve as bridges between theory and practice and they can be understood as having a dual nature.

In the following paragraphs I offer a brief account of two attempts to account for this intuitive inbetweenness of computer simulations, namely those of Johannes Lenhard (2007) and Margaret Morrison (2015) and show that the features they seek to explain by inadequate - misguided comparisons with theoretical or experimental frameworks are better understood when we understand them as features that emerge from computer simulations in virtue of them being members of the type of technical artifacts we call instruments. This intuitive in-betweenness of computer simulations is rather the product of their ontological and epistemic independence and not of what is often taken to be a subservient character.

Consider the view of Johannes Lenhard (2007). In trying to account for this 'in betweenness' of computer simulations Lenhard acknowledges that using them "makes it necessary to run through a process of repeated reciprocal comparisons between experiment and model" (Lenhard, 2007 
p.182). This process of benchmark calibration (Winsberg, 2010) and or validation (Durán, 2018) is, according to Lenhard's view, a way of fleshing out the bridge-like nature of computer simulations: as that which allows for, enables, or that with which we can automate the process of running multiple tests on the fit between the models and the empirical observations. In the context of philosophy of science, Lenhard puts it the following way: "one could say that the traditional hypothetical-deductive testing of model assumptions is transformed into a quasiempirical process. Hence, simulation introduces a new methodology of modeling that opens up a specific perspective on how models manage to be both autonomous and mediators" (Lenhard, 2007 p. 182) The mediating relationship that Lenhard is alluding to is also not in a single direction from theory to model to simulations to empirical observation. In some instances of simulation, in fact, such as in those used for early atmospheric dynamics (Phillips, 1956), it is the exploration of the simulation model — in what Lenhard calls a quasi-empirical way ${ }^{61}$ — that "first made it possible to make a theoretical model the object of research" (Lenhard, 2007 p.181) Thus, Lenhard suggests, the reason why computer simulations are mediators is because they allow model formation to take place through a hypothetical analysis: construct a model, run it (and or a myriad of variations of it) in a simulation and then compare the model's attributes in relation to the known features of the target system, fine tune, and then repeat.

\footnotetext{
${ }^{61}$ It is important to note that this is hardly an exclusive feature of the way models are designed. Simon, for example, says that whenever we are thinking of any artifact we have to "think of the design process as involving, first, the generation of alternatives and, then, the testing of these alternatives against a whole array of requirements and constraints." (Simon, 1996)
} 
Lenhard uses this description to explain the peculiar — and possibly novel—role of computer simulations as a method of inquiry. Computer simulations are experiments on models and in some successful instances allow us to understand the model and the target system by automating the testing of one against the other. In the early days of the advent of the computer in scientific inquiry, for example, the device itself was seen as an "inductive machine" (Dalmedico, 2001). This was suggested when the machine was seen as being able to carry an argument implementing the laws of motion in physics to its logical conclusion, making possible " the making and the testing of physical hypotheses in a field where controlled experiment is still visionary and model experiment difficult, and so permits a wider use of inductive methods" (Charney, 1954 p.153 as quoted by Dalmedico, 2001 p.405).

Similarly, following her analysis of conceptual models as mediators (Morgan and Morrison, 1999), Margaret Morrison (2015) suggests that we think of computer simulation practices as being constituted by both a device and the target of our investigation (2015 p.219). As such we can understand the computer simulation as running strictly numerical tasks (experiments) or computing a formal model's specification and then empirically analyzing the behavior of the implemented model. The target of our investigation in this view is not the original target of the model, but rather the model itself or the properties of the many variations and iterations as they unfold through simulation processes. The simulation as a device, according to her, "allows us to create the kind of controlled environment where one can vary initial conditions, values of parameters, and so on" (2015 p. 219) and test these variations against requirements specified by the nature of our inquiry. Yet again, here we can see the subservient aspect of computer simulations as merely model-building/model-testing extensions of conceptual endeavors. 
In the context of a broader picture of knowledge acquisition and scientific inquiry, any account of the epistemic role of computer simulations must grapple with the questions about what kind of knowledge can they furnish (a priori/a posteriori) as well as with questions regarding how that knowledge is acquired and/or justified (Barberousse and Vorms, 2014; Symons and Alvarado, 2016; 2019; Durán, 2018) The views above, particularly that of Lenhard, position computer simulations as a device that explores theoretical postulates in an empirical way. Similarly, as we will see below, Morrison thinks that computer simulations provide novel insight into the workings of conceptual constructions (models). In the context of the simulation pipeline which I will show in detail in chapter 4, Morrison's view is that computer simulations do in fact bear some similarity relation to a target system. ${ }^{62}$ Yet in the broader context mentioned above, this alone is insufficient to account for whether or not computer simulations relate to the world itself and how they do so. For Morrison, this can be done by treating them as an experiment in measurement practices. That is, for her, when it comes to simulation what we are doing is rather

${ }^{62}$ In the pipeline depiction of a simulation process in chapter 4, the process by which a computer simulation comes together is a series of stages that begin by some observation of a target system, a phenomenon in the world. Once this observation happens the next stage is to abstract some relevant or salient properties of that target system and build a model. As we will see, some depictions of the pipeline acknowledge that the computational elements of the computer simulation are removed by one or two steps from the target system. These intermediary steps can be the mathematical models, or the computational specifications of those mathematical models, or both. In most cases, there is an acknowledgement that there must be some sense of similarity relations at least between some subsequent stages: the mathematical model should bear some similarity relation to the target system, and the computational model should bear some similarity relation to the mathematical model. What Morrison suggests is that insofar as the mathematical model captures the adequate value transformations observed in the target system and the computational models captures the value transformations specified in the mathematical model, then both have a structural similarity to the target system. This is a non-trivial claim concerning the relationship of a model to a target system as well as the relevant epistemic elements of real-world phenomena and our ability to capture them through formal abstractions. However, this is slightly beyond the scope of this dissertation and does not do much for our present discussion. As we will see in sections below, Morrison's strategy also has some features of relevance to the overall discussion of the nature of computer simulations as experiments and these will be treated in detail. 
like the otherwise 'manual' measuring practices of comparing theoretical values (initial conditions, system parameters, etc.), which are often found in scientific practices related to particle physics. Though one cannot have immediate access to subatomic phenomena, one can draw inferences from the comparison of theoretical values produced by the detection of quantitative properties of indirectly related phenomena (e.g. what happens when we use a cloud chamber to infer the presence of subatomic particles). Similarly, computer simulations are basically the automation of these procedures in measurement, namely the tracking of the development of compared theoretical values.

This last point concerning the kind of work that computer simulations perform, namely the controlled variation of initial conditions and values of parameters, is what leads Morrison to suggest what I take to be one of the most sophisticated strategies to understand the seeming 'inbetweenness' of computer simulations in scientific inquiry. In her book, Reconstructing Reality (2015), Morrison states that at least some perfectly established and recognized experimental practices - particularly those in which direct causal intervention with a phenomenon is not possible - consist of nothing more than the observation of the changes of a system strictly through the comparison and manipulation of theoretical values such as initial conditions, value parameters and other indirect means stemming from modeling techniques and not from the target phenomenon itself. If this is the case, and this is also what computer simulations do, then there is no reason to think that computer simulations are epistemically inferior to established experimental practices. Furthermore, if the aforementioned established experimental practices are capable of yielding empirical insight (which would be hard to deny given the general 
epistemic status enjoyed by scientific disciplines such as astrophysics and particle physics), then computer simulations should be thought of as doing so too.

A key aspect of Morrison's argument regarding the status of computer simulations vis-à-vis the status of experiments as empirical practices is the specific kind of experiment that she has in mind. For her, it is key to acknowledge that, contrary to common conception of experimental practice, there are experiments that strictly focus on tracking variations of initial conditions and comparing behavior of theoretical values. These kinds of experiments are like other activities in inquiry related to measurements, which rely to a greater degree in formal aspects (theoretical values, principled equations, etc.) of inquiry and not on material manipulations of phenomena. If computer simulations are like experiments, she argues, they are like these kinds of experiments. To her, measurement involves "some functional relation to instruments that generate data from some physical source. In that sense, a measurement, even one that is indirect, bears some type of connection to the property being measured" (Morrison, 2015 p.236) As an example, Morrison uses the manipulation of physical models by $19^{\text {th }}$ century British scientist to determine features of or quantities in electromagnetic fields. This led to the modification of Maxwell's equations (Morrison, 2015, p.203). This is important because it signals that experiments which are focused on theoretical values and/or principled elements of inquiry can and do provide useful knowledge that in turn can be used to fine tune either the empirical experiments (conventionally understood) or the theories upon which these are based. This is a claim that directly challenges those that think that formal elements of inquiry cannot provide information beyond whatever is already implied a priori. As an example, Morrison cites the physical plane pendulum to measure local gravitational acceleration (p.203). In this case, she says, "the model operated as a device that 
was, at least in the hands of Kelvin, able to measure certain quantities.” (p.203). If this is so and computer simulations can be understood as carrying out this same sort of task, namely manipulation of theoretical values extracted as data from some other experimental practice, then computer simulations are just like measuring practices, particularly those carried out in contexts of high-energy physics and other branches of science in which the analysis of a phenomena is comprised of many indirect formal steps. According to her, the connection between models and measurements "extends beyond the theoretical interpretation of outputs to an understanding of the apparatus itself and how the representation of physical phenomena 'embedded' in the model/instrument enables us to use it as a measuring device." (2015). That is, models aren’t merely maps to follow paths of deductive inference rooted in the kind of a priori assumptions and laws found in scientific theories. Rather, she says, "models are experimental tools capable of functioning as measuring instruments" (Morrison, 2015 p.228). For her, this last point explains the in-betweenness, the moderator aspect, of computer simulations (Morgan and Morrison, 1999; Morrison, 2009; 2015). According to her, they are the kind of device, that just like a measuring instrument, is strongly reliant on theoretical values whose internal cohesion can be investigated by the manipulation of numerical values alone without the need for direct external causal manipulation of a phenomenon. After all, it is not likely that we will soon intervene with the workings of a star or a similarly unreachable/impenetrable system, and yet, through measurements of the kind just described above we are sufficiently confident that researchers are managing some sort of inquiry about them.

All of the above may sound rather promising for the instrument view I am defending here. However, closer inspection reveals that the terms such as 'devise', 'artifact', or 'measuring 
instrument' are used rather as mere placeholders for yet another account of abstract methods of inquiry and not to refer to any aspect of the necessary material implementation of such. They are being used almost as metaphors. This is in sharp contrast to what the instrument view of computer simulations is designed to do. Nevertheless, for now, I will focus on other problems with the views described above. There are two immediate problems with Morrison's strategy for explaining the seemingly ambiguous epistemic role of computer simulations in inquiry. The first one is that Morrison's view of experiments here is a deflationary one, this is in contrast to earlier views in the philosophy of computer simulations which sought to inflate the status of computer simulations to understand them as closer to those practices that can provide empirical knowledge in virtue of their necessary materiality ${ }^{63}$. So, it is not so much that she is bringing computer simulations upwards onto the status of experimental practice but rather that she is choosing to focus on a broader notion of experiments which include processes such as measurement. ${ }^{64}$ The second problem is that once we understand that the kind of experimental practice Morrison is comparing computer simulations to is a deflationary one, her view corresponds more closely to the narrow views of computer simulations rather than to the broad views we saw in Chapter 1. This is particularly the case when she suggests that there is a continuous structural similarity across the multiple stages required to get to a computer simulation from the salient features of a target system. This is a problem because the many non-trivial transformations required for purely mathematical elements to become part of

\footnotetext{
${ }^{63}$ See Parker (2009) for a thorough account of the 'materiality thesis'. Briefly, the materiality thesis posits that the necessary physical implementation of computational processes is sufficient to claim that processes such as computer simulations are indeed manipulating and causally connected to phenomena in the world. If this is the case, then this cannot be a differentiating factor between conventional experiments and computer simulations.

${ }^{64}$ The distinction between the kind of inquiry that causally manipulates aspects of the world and that which only manipulates abstract content goes back to the earliest days of science. More details on this in footnote 59 below.
} 
a computer simulation are simply glossed over. So are the many extra-mathematical elements of computer simulations we have extensively covered here. More importantly, so is the necessary artifact that makes all of the processes described above possible: the concrete computational devices instilled with the digital specifications to simulate.

In order to better understand these issues, let us begin by looking at the first problem in detail. As mentioned above, Morrison's view provides a rather unorthodox answer to one of the questions her view is designed to address: "if we identify simulation with modeling, then how can we account for the experimental features typically associated with simulation?” (Morrison, 2015 p.227) Her answer to the question is to redefine experiments in a way in which at least some of them are not fully-fledged empirical practices but rather manipulations of theoretical constructs and values. It is important to notice that this is in line with some of those that view computer simulations as nothing more than extensions of formal methods such as mathematical equations and conceptual models. ${ }^{65}$ For Morrison, simulation models are basically "the result of applying a particular kind of discretization to the theoretical/mathematical model" (p.219). The computer program allows us, according to her to "investigate the evolution of the model physical system." Insofar as the simulation system is computing the relationship between theoretical values and their evolution,

${ }^{65}$ Weisberg (2013), for example, thinks there is a sense in which questioning the model-world relationship in the context of computational simulation is a misguided question. This is because "models are not compared directly to real phenomena, but to target systems" instead (2013). Target systems, in turn, are formal abstractions (2013, p.95). This abstraction comes in the form of measurable components that make it to the model in the form of weighted variables. According to Weisberg, these variables correspond to quantities. Target systems are made of measurable variables..$^{65}$ Thus they are both abstract mathematical objects and can be compared to each other without much worry of crossing ontological boundaries between formal properties and empirical properties (p.95). 
then it is like performing an experiment. Further, if some experiments are merely interventions on measured variables and the measured variables are the product of the theoretical model, then there is no clear distinction between them and computer simulations (2015). She argues that computational methods simulating systems of particles are particularly suited for this kind of description. And she contends their importance to her view is that "their computational structure involves a large number of abstractions that help in their computational implementation", while at the same time being "inherently linked to the physics they simulate." (Morrison, 2015 p. 223) In other words she argues that at least in these particular kinds of models the relevant features of the physical system can be captured by mathematical characterizations of their attributes such as mass, charge, etc. (2015, p.221) However, just as in Weisberg's view, described above, the relationships here mentioned are relationships that hold between abstract entities. That is, what she calls the simulation model (which is the machine-readable translation of the mathematical model) is nothing but the application of discrete mathematics to another kind of mathematical or theoretical model.

Furthermore, the computer program that allows for the "investigation of the evolution" of the simulation model is just that, namely a program. Which to her is just a series of logical specifications in a formal language to carry out discrete numerical operations. For Morrison, in short, a simulation is an abstraction of an abstraction of an abstraction. None of which ever allude to the implementational features of an independent, material artifact. Hence Morrison's position here can be understood as a deflationary view of experiments rather than elevation of computer simulations towards similar epistemic status of empirical practices. Her view is not so much to equate computer simulations and experimental practice in virtue of the fact that computer simulations may have access to or can provide the kind of empirical knowledge often thought to 
emerge from conventional experimental practices but rather to suggest that many experimental practices are nothing more than manipulations of theoretical values and investigations on the behavior of models of target systems and not the systems themselves. ${ }^{66}$ This deflationary view may prove disappointing to those whose primary goal in claiming computer simulations are closer to experiments than to theory is to account for the possibility that computer simulations could provide novel, a posteriori, empirical knowledge - a view that though often defended by practitioners and those with pragmatic intuitions nevertheless remains strongly debated in the philosophical literature.

While there are many important questions raised by Morrison's strategy that are beyond the scope of this work in the context of our present discussion, her position can be understood to indirectly side with the view of computer simulations that puts them closer to theoretical constructs. Morrison's view sidesteps a thorough discussion of the artifactual nature of computer simulations. And this is the second of the two problems discussed above. Despite Morrison's

${ }^{66}$ In a sense, the problem with this deflationary account is analogous to a very old distinction between what were called mathematical and philosophical instruments. The distinction between what was thought of as models and instruments - or philosophical apparatus, as they were called — and a discussion of them can be found discussed as early as 1649 in correspondence between Boyle and Bacon (Warner, 1990 p.83) Even without the complexities introduced by computational methods, the history of the notion of instrument in scientific inquiry has its ambiguities. The term, for example, was not used before the 1800's (Warner, 1990). Before then, the devices surrounding what we now understand as practical methods of hypothesis demonstration were called philosophical instruments, whereas those associated with measuring were called mathematical instruments. The key distinction here is between the kinds of artifacts deemed to furnish information about the world and those that were deemed to be merely a product of an arbitrary formal construct. The distinction between philosophical instruments and 'mere' mathematical instruments is similar to the distinction between those instruments that have direct, what we now understand as empirical, access to an aspect of the world and those that are constituted of and solely manipulate mere formal conventions. If this distinction holds then Morrison's account of computer simulations is that of a mathematical instrument. That is, an instrument that does not have nor can provide access to phenomena in the world. 
suggestion that computer simulations can function "like a piece of laboratory equipment, used to measure and manipulate physical phenomena" (2015 p. 219), the actual physical aspect of their functioning is not explored and is overshadowed by the characterization of their functional specifications as a mere product of the abstract side of their nature.

Lenhard's strategy is not significantly different from that of Morrison. In order to do, as he says, more than merely "reconcile theory with experiment" through the use of computer simulation, he posits that computer simulations consist of a methodological cooperation (p.177) in which the novel feature is the subservient role that simulations play in enhancing (automating, accelerating) modeling techniques. This is important to him, because this approach helps him make the case that computer simulations are more than mere mathematical machines while keeping the intuitions about their experimental features intact: a computer simulation, in his view, is like a modeling device with which to conduct theoretical experiments (Edmonds and Hales, 2005). In so doing Lenhard thinks that he explains the in-between status of computer simulations.

The extent to which computer simulations, by themselves, have been characterized as a completely novel and independent element of scientific inquiry — at times being touted as the equivalent of a scientific revolution in itself — in virtue of the ambiguities and the hybridity of their place in scientific inquiry can be seen with the following example. Paul Humphreys (2004 p.51) lists three different excerpts from "internal commentators" on the epistemic impact of computer simulations in science. I include Humphreys' original quotations almost in full because I believe these excerpts fully exemplify the nuances of the landscape of interpretations of the role of computer simulations in science that my position aims to break away from. In particular, my 
view aims to break away from the view that computer simulations are in and of themselves a third branch on inquiry, which neglects the fact that if they indeed partake in a third methodological approach it is because of their membership in the broad set of things we call instruments, as I will argue below. Though these views are from practitioners, I believe they reflect the same spirit encompassed by the philosophical approaches described above. The first one is from Physics Today (1984) and says the following:

"Science is undergoing a structural transition from two broad methodologies to three — namely from experimental and theoretical science to include the additional category of computational and informational science." (Nieuwpoort, 1985 as quoted in Humphreys, 2004 p.51)

The second remark addresses the 'in-betweenness' I have been discussing in this section and is subtly a bit less contentious in that the author takes care to qualify the statement by noting that computer simulations may not be the only methodology that elicit this bridge-like feature in scientific inquiry:

“Computer simulation provides (though not exclusively) a qualitative new and different methodology for the physical sciences, and that this methodology lies somewhere intermediate between traditional theoretical physical science and its empirical methods of experimentation and observation" (Rohrlich, 1991, p.507 as cited in Humphreys, 2004 p. 51) 
And lastly the following third comment casually but boldly reflects the ingrained neglect of the role of instrumentation in scientific inquiry throughout history:

"For nearly four centuries, science has been progressing primarily through the application of two distinct methodologies: experiment and theory... The development of digital computers has transformed the pursuit of science because it has given rise to a third methodology: computer simulations." (Kaufmann and Smarr, 1993, p.4 as quoted in Humphreys, 2004 p.51)

Here we have three descriptions of what the introduction of computational methods, particularly computer simulations, have done to science and what they represent in science. The consensus, within these three descriptions, is that computer simulations constitute a third, novel methodological element of scientific practice. Humphreys notes that these characterizations may prove too strong and he criticizes them accordingly. Yet, his criticisms miss the fact that there has been, for a long time, a third branch of science already at play and that is exemplified by the instruments we often require to even conduct theoretical or experimental endeavors. He mentions three possible objections to the boldness of the claims above: 1) you could say that computer simulations are just extensions of numerical methods already in existence; 2) computer techniques do not qualify as a method apart because they do not directly access empirical content; and 3) examining the reliability of computational methods has serious-enough challenges that may prevent them from ever meeting the requirements of rigor in scientific 
settings. ${ }^{67}$ Humphreys goes into detail in each of these objections and further points to some misunderstandings within them, but the point here is that at no time does Humphreys mention that this "new" "structural transition" to which these authors refer to was not new at all, or that their supposed "third methodological category" was either always there or, if not, had at least already happened in physics centuries prior to the advent of computers and in chemistry at least decades before - its importance already widely documented, at least in the history of science (Van Helden, 1994; Harré, 2003; Kroes, 2003; Baird, 2003; 2004). In fact, this distinctive role of instruments as well as their dual nature as physical and epistemic enhancers has been documented as far back as Bacon when he stated that "Neither the naked hand nor the understanding left to itself can effect much. It is by instruments and help that the work is done, which are as much wanted for the understanding as for the hand." (Bacon, 1965 as cited by Van Helden and Hankins, 1994 p.4) Humphreys does not regard computer simulations as instruments because he views instruments mainly as "property detectors" (2004 p.28). Because of this, however, many precision instruments deployed in science will simply be left out of Humphreys' characterization. Consider shafts and gears as an example.

The three quotes above are notable insofar as they show the conventional understanding of both scientific endeavors and of the role of computers in scientific inquiry; the two projects discussed above — that of Morrison and that of Lenhard - are a sample of the many attempts by

${ }^{67}$ This third objection is quite interesting as it is a strong and valid objection against the widespread and indiscriminate use of computer simulations in scientific inquiry, not as a third category of methodology all to themselves, but rather as members of the exclusive club of technical artifacts that we deem reliable enough to be called 'scientific instruments'. This point will be discussed in the final section of this dissertation. 
philosophers to make sense of the ideas expressed in these quotations. Attempts at explaining the in-betweenness and the apparent novelty of computer simulations and their role in scientific inquiry have usually taken the form of an appeal to an unprecedented third way of doing science altogether (Resch et al, 2017). However, there is a simpler and more general explanation to why computer simulations are often found to be at the epistemic intersection of theory and experiment, or why they can be understood as measurement devices, or why they seem to have an ambiguous epistemic status as almost-models and/or almost-experiments: it is because they are technical artifacts, which elicit all these properties: a dual nature — as the physically implementation of a conceptual design with a function; as measuring devices that can deploy modelled theoretical values and contrast them with data (real or simulated); and finally as 'almost theoretical' and 'almost experimental' — because they are neither. They are instruments and, as I will argue following the views of Davis Baird (2004), instruments are the third element of scientific inquiry.

The philosophical views above, including Humphreys', shed light on aspects of the question. Both Lenhard's and Morrison's attempts to account for the seemingly ambiguous epistemic role and position of computer simulations in fact_-inadvertently_capture an important aspect of computer simulations as technical artifacts, artifacts that necessarily have a dual nature (Kroes, 2003) - as physical implementations of functional specifications with conceptual, intentional origins (Symons, 2010). What the views described above are missing is a broader picture in which computer simulations are in between theory and experiment in virtue of belonging to yet another class of objects with independent epistemic properties from theoretical and experimental elements of inquiry: namely, they belong to the class of objects, more precisely artifacts, we call 
precision instruments. This in betweenness, ambiguity if you will, of computer simulations which we just analyzed in detail through Lenhard's and Morrison's attempts at reconciliation can be easily explained — or even explained away—by understanding that computer simulations are first and foremost technological artifacts and that technological artifacts are, as stated many times before, of a dual nature (Kroes, 2003). ${ }^{68}$ So, let us now look into this a little bit more in detail.

An intuitive way to individuate an artifact is by its relation to some agential intentionality (Kroes, 2003; Symons, 2010). ${ }^{69}$ Artifacts come in many different shapes and from many different sources. Some are artifacts solely in virtue of already having a desired property when encountered, others are made to have a desired property in virtue of their design and materiality. The latter are the more definite artifacts. They do not only have some artifactually advantageous property as found but are explicitly designed and constructed to have that property. ${ }^{70}$ Artifacts in general, but technical artifacts in particular, due to their relationship with agential intentionality can be said to be the marriage of two ontologically distinct sets of properties:

specified/defined/expected functions and the physical elements that can instantiate them. Herbert Simon, for example recognizes that artifacts are interfaces, or a "meeting point $[\ldots]$ between an 'inner' environment, the substance and organization of the artifact itself, and an 'outer'

\footnotetext{
${ }^{68}$ Whether by gears or electrons, but physically executed nonetheless.

${ }^{69}$ While there are many issues regarding this intuition for the purposes of the present discussion, this is a goodenough way to tell objects apart.

${ }^{70}$ Kroes calls the former pseudo-artifacts in virtue of the fact that they are only circumstantially artifactual and not intentionally so.
} 
environment, the surroundings in which it operates." (Simon, 1969). Technical/technological artifacts are those which are explicitly, by design, constructed with an inherent practical function (Kroes, 2003 p.69). As such, in order to fully account for them (i.e. individuate them, describe them, understand them, etc.) both their physical properties and their teleological properties must be included.

This explains, to a certain extent the recurring intuition that computer simulations have something of a dual ontological nature between theoretical and empirical practices: they are the product of conceptual specifications and the physical implementation of the functional character of these specifications. In other words, their nature as technical artifacts further explains the ontological independence, discussed above, in a way that makes sense of their formal underpinnings and their necessary materiality as the things with which the teleological specifications are instantiated.

The epistemic in betweenness of computer simulations also arises from the fact that they are technical artifacts, but the details surrounding this aspect of computer simulations is different from those concerning epistemic independence. Instruments, such as the ones used in science, are highly specialized technical artifacts. When they are well made, they are constituted by highly theoretical and functional specifications as well as by very specific material which is optimal for the execution of the functional specifications in light of theoretical requirements (Symons and Alvarado, 2019). This much constitutes their ontological independence: instruments are highly specialized technical artifacts that are the product of sophisticated functional specifications and sophisticated material properties to carry them out. Yet, once they 
are made, these highly specialized technical artifacts are taken in to the laboratory setting where they are deployed to perform a role in the acquisition of knowledge. It is there that technical artifacts are further placed somewhere between theory and experiment. This is yet another level of consideration from that which we have been discussing. And here too, instruments such as computer simulations show a kind of conceptual independence: as they are made, they are not strictly members of theory or experimental practice; as they are deployed, they are not so either. The relationship between the specific instrument and the experimental setting or the theoretical underpinnings of the specific inquiry can be very diverse, as diverse as the number of scientific instruments out there. Therefore, it would be misguided to attempt a general description here of the nature of this relationship. Rather, consider the following. One of the reasons why there is a relationship in the first place between one and the other is because instruments are not theory or experiments, they are a third distinct thing. That is, there is a relationship between those other elements of inquiry and instruments precisely because instruments are an independent element of inquiry. ${ }^{71}$ The ontological independence together with the functions that these instruments carry out beyond those of the theoretical and experimental functions is evidence that they are also epistemically independent. As we will see, instruments can serve distinct epistemic tasks but they can also consist of independent epistemic sources. More strongly put: instruments are a

\footnotetext{
${ }^{71}$ Of course, some things can have a relationship to themselves: identity. The basic conceptual claim here is that instruments are non-identical to the other elements of inquiry. Practically speaking, the reason why there are still questions about the role of instruments in relation to theory and/or questions regarding the role of instruments in relation to experiments is because instruments are not strictly speaking identical to the theories or to the experiments they are related to. For a full analysis on the independent ontological and epistemic status of instruments see Baird (2004). For a thorough analysis of some of the non-trivial contributions of instrumentation to scientific inquiry see Hacking (1983)
} 
third — separate, distinct, and independent) — source of knowledge in scientific inquiry (Baird, 2004).

According to Baird, instruments cannot be understood in terms similar to those with which we understand theories because they are not constituted of the same things (2004, p.4). That is, if theories are the systematic and coherent collection of propositional knowledge then instruments, by virtue of not being propositions themselves but something more than propositions cannot be understood solely though the same articulations we understand theories with. Hence, the life of an instrument, as such, requires its own epistemological account independent from the one for theory. (Baird, 2004) This explains why they don't fit on either side though they can carry out functions associated with both. This explains too why computer simulations, as instruments deployed in scientific inquiry, elicit all these features that make them be seen as in between. They are ontologically and epistemologically separate and distinct from both theory and experiment.

So, whether the view is that computer simulations function as aids to some sort of deflated empirical/experimental practice such as measuring devices or whether one thinks of computer simulations as something in between, these features, capacities and roles of computer simulations can in fact be better understood if we understand computer simulations as instruments. 


\subsection{Computer Simulations in Science}

As stated above, observations that computer simulations are somewhere in between theoretical concepts and empirical practice - or that they act as moderators between them, or even that they are measuring devices - have in fact done a great deal in moving the debate forward and enriching our understanding of the role computer simulations play in scientific inquiry. These views manage to capture something essential about the epistemic nature and independence of computer simulations in relation to these established elements of scientific inquiry. However, what is lacking in these views is the full acknowledgement that these properties of computer simulations (i.e. their dual nature as product of the physical implementation of abstract functional design and their simultaneous epistemic independence) happen in virtue of them belonging to a larger class of things, namely technical artifacts. Furthermore, computer simulations are not just any technical artifact, like a car or a bicycle. Rather they are produced in the context of what is thought to be a highly sophisticated knowledge-acquisitions context: scientific practice. In short, computer simulations, as technical artifacts, are deployed as instruments in science and their adequacy as such should be understood in the same terms. There are complex and sophisticated artifacts that, despite their complexity and sophistication, fail to meet scientific standards. There are complex and sophisticated methods of analysis, such as data mining, that nevertheless fail to meet norms and criteria for deployment in scientific inquiry. So far, what this means is that while computer simulations are an instrument, it does not automatically mean that they are a scientific instrument. This also means that just because an instrument has been deployed in scientific settings it does not mean that it should be so deployed. 
So far the view I have defended in the previous sections goes something like the following: some things are technical artifacts, this is particularly the case with those things that are intentional physical constructs with a dual nature between functional specifications and the materiality required for their execution; out of these technical artifacts a few highly sophisticated ones are designed and developed with the aim to be deployed in scientific inquiry. Those technical artifacts that are deployed in science — specifically the ones that have been sanctioned and vetted through the rigorous epistemic requirements of scientific practice ${ }^{72}$ - are the ones we call scientific instruments. Computer simulations are - perhaps for historical reasons — often thought of as belonging to this latter kind. Whether they should be is a question that will be considered in the last section of this dissertation. In the paragraphs immediately below, I focus on the following question: if computer simulations are the kinds of technical artifacts that are deployed as instruments in science, what kind of instrument are they and what do they do as such?

As a preliminary step it is important to note that not every artifact that is deployed in a scientific setting is a scientific instrument, even if that artifact is a technical artifact. Further, conceptually, not every artifact that is deployed in a scientific setting has what it takes to be a scientific instrument. Some fail. Others weren't meant to be a scientific instrument in the first place.

\footnotetext{
${ }^{72}$ As I will make clear in detail in chapter 5, particularly section 5.3.1, scientific standards are, and should be, of a stricter kind than those required of us for everyday epistemic practices. In fact, scientific epistemic standards and norms are such that if we attempted to switch one for the other we would in effect be undermining each of the practices involved: if I apply epistemic requirements typically associated with science in my everyday activities chances are that those activities will not get done; if I apply everyday epistemic standards in scientific practice, it would be difficult to justify exactly how it is that science is still being done. This is particularly the case if the distinctive aspect of science, as an epistemic practice, is defined by reference to anything involving its methodology and the procedures by which such is implemented.
} 
Consider for example the humble light switch inside the laboratory. While it is deployed in a scientific context, the laboratory, and in fact does help those that are carrying out scientific inquiry within the laboratory, the light switch — considering it is of the generic kind easily found at hardware stores - as such is not intended to fulfill a function with scientific specifications. ${ }^{73}$ This intentional aspect is worth examining with a bit more detail. John Symons (2010), for example, makes an important distinction between an object like an organism that has something like a set of proper functions and an object that is designed with a function. With this distinction, he suggests, functionally individuated objects such as organisms and artifacts can be told apart: both carry out epistemically identifiable functions but one was intentionally designed to do so. This is similar to a distinction marked by Peter Kroes (2003) who suggests that while some intentionally designed functional objects qualify as a technical artifacts, those which can carry a function only circumstantially - that is, in virtue of having a 'natural' property that can be appropriated for some uses - are only technological pseudoartifacts (p.69). According to him, the latter do have/carry out a practical function and that is why they can still qualify as 'technological' but they do not do so in virtue of an agential intention and that makes them less of an artifact. $^{74}$

\footnotetext{
${ }^{73}$ As we will see, scientific specifications require an instrument and/or method to satisfy stricter epistemic criteria than those required for ordinary epistemic practice. As I will also show in the sections below, this will also be a challenge for the deployment of computer simulations in scientific practice: though they may be intended for scientific purposes, their design and development sometimes fail to meet some of these stricter epistemic requirements.

${ }^{74}$ Kroes grounds this intentional character of technical artifacts in terms of human intention. However, one may easily imagine another kind of agent with intention capable of producing an artifact with a specified technical task. An interesting question however may be whether agents that are seen as having inferior technological capacities are ever capable of making artifacts in Kroes' view.
} 
These two distinctions serve as the basis for what I am about to suggest. Intentionality, it seems, matters in differentiating an artifact from a pseudoartifact as well as from other functionally individuated objects. Due to the level of sophistication and rigor that most scientific endeavors have historically attained, or rather - as I would argue - are normatively required to exhibit, the intentionality of the functional aspect of the technical artifacts deployed in science is also of a different nature than that of a technical artifact deployed in another context. That is, the design specifications for an artifact used in science will be different from those for an artifact used for less epistemically rigorous settings. Because there are normative differences in the way inquiry is conducted in science, then the intentional functionality of instruments designed, developed and deployed in science is also different.

There is a higher-order intentionality when it comes to developing technical artifacts meant to be utilized as scientific instruments, namely that they meet the standards implicit in epistemic norms of scientific inquiry. The reason why instruments in science must be precise is not merely due to an arbitrary obsession with minuscule metrics on the part of scientists. Rather, the intentionality behind precision falls from the need to document in exact terms so that norms such as reproducibility are met. The insistence on reproducibility stems from the idea that a single observation is insufficient to derive reliable conclusions. In other words, scientific instruments must meet the higher epistemic norms of scientific inquiry because science itself is designed with the aim to overcome conventional epistemic practice. There is, in fact a normative difference between technical artifacts in general and those designed, developed and deployed in scientific inquiry. 
Whether these normative differences are independently normative in themselves or are a historical element of the development of science is yet another question. However, for my present purposes it suffices to say that the evidence that these distinction in normative requirements between scientific and non-scientific settings are in place is the existence of a centuries-old precision instrument industry (Heilbron, 1993). ${ }^{75}$ This industry was born out of the need — or desire ${ }^{76}$ — for more reliable and precise instrumentation. And it signals that the intentions with which artifacts deployed in scientific inquiry are different than those for the artifacts deployed elsewhere, even if only in relation to the higher-order requirements of the methodology, such as precision. The intention when constructing a scientific instrument is not merely to construct a technical artifact but rather to construct an artifact in accordance with scientific expectations. In other words, if the intention is to construct an artifact to satisfy a scientific function, this will be reflected in its design. This will prove very important when I move on to assess whether or not computer simulations ought to be so ubiquitously used and accepted in scientific inquiry (Chapter 5). For now, this distinction had to be made in order to position computer simulations as the kind of technical artifact that is used as, or is on its way to being deemed, a quintessential scientific instrument.

\footnotetext{
${ }^{75}$ That there is evidence for a stricter set of epistemic requirements in scientific endeavors will also prove important for later sections in which I discuss the epistemic challenges in assessing the reliability of computer simulations.

${ }^{76}$ Even if this aim for a more reliability and precision was born out of a stubborn desire from those that practice science, this is evidence that there is an intentional aim that informs the method and drives its design. Hence the higher-order requirements for any instrument that is to partake in this particularly rigorous practice.
} 


\subsection{Instrument Taxonomies and Computer Simulations}

I have argued that computer simulations are ontologically distinct, epistemically independent and that some of their most contentious features are best explained when they are understood as the kinds of technical artifacts we call scientific instruments, now we can move on to discussing what kind of technical artifacts they are and what it is they do. It would be a futile exercise to try and provide a detailed taxonomy of the specific functions and uses of computer simulations in scientific inquiry as there are just too many examples. Rather, here I discuss a bird's eye view of their relation to other artifacts as well as to the general enterprise of scientific inquiry as a knowledge-gathering practice.

As I showed in the sections above, part of the in betweenness of computer simulations comes directly from the fact that they are technical artifacts, which are already the kinds of objects with a dual nature. Computer simulations, as separate, distinct and epistemically independent devices, also offer researchers an inexpensive, automated, alternative to conduct explorative procedures on both experimental practice and theoretical elements of inquiry. As Morrison suggests, computer simulations provide a way to automate the provisos of a controlled experimental setting and hence also the ability to play around with variations on parameters, initial conditions, as well as other theoretical values and transformations. As such they provide a way not only to run experiments or run models but also to investigate the adequacy and/or lack thereof of models and experimental settings. This bidirectional feature also makes of computer simulations a hybrid of sorts in that they allow to experiment on the things that inform them-experiment on 
the experiments, if you will. ${ }^{77}$ In this sense they are not just the things with which your run models or experiments but the things with which you can explore the many ways to run a model or an experiment (Lenhard, 2007).

As characterizations of instruments go, computer simulations end up being hybrids in many other taxonomies too. This may indeed be evidence that computer simulations are in fact a novel addition to scientific inquiry, and that they may correspond to a yet unprecedented category as correctly acknowledged by the views of Morrison, Lenhard and Humphreys discussed above. However, simulations may turn to be novel and a category onto themselves within the realm of instrumentation and not amid scientific methodology as a whole. In the following sections I go through three different taxonomies of instrumentation and show how computer simulations, as technical artifacts, as members of scientific instrumentalia (Durán 2018) hover above and in between their classifications. As mentioned above, this further solidifies the intuitions discussed above regarding their in-betweenness and their novelty, but this time within the category to which instruments belong.

First and foremost, computer simulations, as technical artifacts go, belong to the class of technical artifacts whose main function is to serve as epistemic enhancers (Humphreys, 2004). This rough characterization is sufficient to provide an intuitive framework in which we can

\footnotetext{
${ }^{77}$ This is what Lenhard (2007) referred to as the 'quasi-empirical' aspect of simulation modeling and what Morrison (2015) meant when she wrote that computer simulations allowed for 'testing' of both theoretical and experimental stipulation. Herbert Simon, (1996) too, identified the potential for computer simulations to not only provide means to solve an equation but also provide the means to explore the numerous ways in which an equation could be solved.
} 
differentiate them from the kinds of artifacts that enhance other limitations of human agency, such as physical strength. The kind of enhancement that a calculator provides, for example, is different from that of a bulldozer. While in a scientific setting any instrument can be said to contribute to the general aim of knowledge acquisition, we can still differentiate between the artifacts that augment our physical capacities and those that augment our epistemic ones. ${ }^{78}$ If computer simulations enhance anything, they enhance our ability to acquire knowledge and not our ability to push harder or dig deeper. ${ }^{79}$

According to Humphreys, there are three ways an epistemic enhancer can extend the reach of our understanding. The first one is extrapolation, which is the capacity of an instrument to expand "the domain of our existing abilities" (p.4). Then there is conversion which happens when "phenomena that are accessible to one sensory modality [...] are converted into a form accessible to another" (2004 p.4). And finally, there is augmentation. This last kind of enhancement occurs when, mainly through one of the other sorts of enhancements - particularly conversion (p.4) we are "given access to features of the world that we a not naturally equipped to detect in their original form”.

\footnotetext{
${ }^{78}$ There may also be a difference between those artifacts that enhance the means by which we gain understanding and those that directly enhance our understanding, though that is a topic far too removed from the discussion in this work.

${ }^{79}$ There is an important philosophical question concerning what exactly a telescope allows us to do. That is, what it is that telescopes allow us to enhance. For a thorough discussion of whether and how we can actually 'look closer' with a telescope see Hacking (1983)
} 
At first sight, it is easy to take computer simulations to do all three and often at the same time. A careful reading of what Humphreys has in mind, however, reveals that there are some challenges in this characterization. In order to better understand the epistemic role and position of computer simulations it is worth going through these three types of enhancement in detail as they provide a picture of the kinds epistemic endeavors that computer simulations undertake as well as a glimpse into why they are hybrid instruments across many domains and dimensions of inquiry. We can understand each one of these with the help of some examples. Humphreys (2004, begins by pointing to the perceptual enhancement characteristic of optical instruments to exemplify extrapolation. Telescopes and microscopes, for example, expand the domain of the visible things for us or the level of detail of a perceptive ability that most of us are already acquainted with, namely vision. Similarly, other kinds of telescopes expand the range of the spectrum of electromagnetic radiation available to us without them. When it comes to computer simulations, we can see that, at the very least - particularly if we share Humphreys' understanding of them as mathematical machines - they enhance our existing ability of analysis. That is, if we consider that we as epistemic agents have an analytical ability, say to manipulate and entertain the relationship between values as well as of the relationship with the symbols that represent these values and infer from their transformations, then we can see that computer simulation indeed expand on this existing modality.

Conversion is a lot more straightforward and can be exemplified by the translation that musical notation undertakes when implemented on a musical instrument: the information on the sheet of music is of a different kind, namely visual, and has been converted onto a sound. In the scientific context of computer simulations one can immediately see this type of enhancement occurring 
when computed numerical values are converted into pixelated gradients on a grid and the transformations of such values are displayed as spatial changes on a screen. In this example, mathematical, or merely numerical information is transformed into visual information. So, computer simulations also convert. Whether or not computer simulations allow us to augment in the sense specified by Humphreys is an interesting question. Humphreys himself notes that this is not immediately obvious from looking at what computers do. This is particularly the case because of his views of computer simulations as the sort of thing that we use solely for mathematical tasks. In his view, computational methods have not yet proven to have "given us access to features of the world [in this case I assume he refers to mathematical features] that we are not naturally equipped to detect in their original form." This is a contentious issue that I hope to take on elsewhere ${ }^{80}$, for now, it suffices to say that the ability of computer simulations to both extrapolate and convert is evidence of their hybridity as epistemic enhancers. What this shows, at least, is that they are not just one kind of epistemic enhancer but rather that they can have multiple functions and function as multiple kinds of instruments at once.

${ }^{80}$ Humphreys does not believe that computer simulations have the capacity to enhance our capabilities in mathematics: as explained in a footnote earlier, to Humphreys computer simulations solve intractable problems in mathematics. They do this by being faster than us but not by providing us with a novel way of doing mathematics (representational opacity notwithstanding), therefore, if they are epistemic enhancers, they are so only in the sense that they can calculate faster than humans. I find this claim to be somewhat misguided. Novel representational devices have in the past enhanced our access to areas of knowledge not previously available to us. Consider the notation of calculus, or the advent of statistical concepts such as the average. In this sense, the representational capacities of computer simulations may very well be positioned to provide us access to aspects of the world previously unavailable to us, and these aspects may prove to be mathematical at their foundation even though not immediately apparent to us as such. Here I am thinking of neural networks and other similar computational methods in machine learning and statistical analysis. 
On another taxonomy, computer simulations also prove to be hybrids of some sort. They can be more than one kind of instrument. According to Baird (2003 p.45), there are three kinds of instruments: models, which represent; devices that create a phenomenon; and measuring instruments which can either detect the instance of a property or compare theoretical values against a phenomenon. Measurement instruments, as we will see, are, according to Baird, hybrids between the kinds of instruments that represent and those that create or recreate a phenomenon. This is because they must create/recreate a set of procedural steps in order to obtain their reading. Models, according to Baird, are not merely representative in that they 'stand in' place of actual phenomena of interest. Rather, they are representative in that they integrate knowledge and are constituted by knowledge of the target itself in an epistemically independent way. He explains this epistemic independence of models as instruments via Watson's and Crick's double helix DNA model. In this case, Baird says, they “did not use the model as a pedagogic device. They did not simply extract information from it. The model was not part of some intervention in nature. It was also not a part of an experiment." (p.36) And yet, the model had the standard theoretical virtues since "it can be used to make explanations and predictions. It was confirmed by X-ray and other evidence, and it could have been refuted by evidence.” (p.36) Computer simulations a we saw in detail in sections above can function like this when they are used as a device that is independent from both theory and or empirical experimentation to test and/or inform theory or experiment construction (Lenhard, 2007). Furthermore, they are often designed with their representative functions in mind. Some simulations, like those of cellular automata are paradigmatic of the dynamic Baird is alluding to. They were developed independently of any theoretical framework associated with any particular phenomenon, or even discipline. They were also developed independently of any particular 
experimental setting associated with an inquiry onto a target phenomenon. While they were themselves experimental, they were not part of a premeditated focus inquiry besides that of investigating the features of the machines that produced them. It was only later that they came to be used as a tool that could to provide both theoretical and experimental insight regarding the formation and development of systems deems to be similar enough to them.

Measuring instruments on the other hand, according to Baird, work by generating a signal from an interaction with a given target "which, suitably transformed, can then be understood as information about" that target (Baird, 2004). According to Baird, measurement requires that we can "produce, in laboratory conditions, a stable numerical phenomenon over which one has remarkable control.” (Hacking, 1983, as cited in Baird, 2004) Measuring instruments are instrumentally encapsulated knowledge (Baird, 2004 p.68) and are constituted by the integration of a material object and the kind of knowledge provided by a model. Hence, measuring instruments are hybrids in that they must reproduce, perform, a set of specified procedures in order to represent, signal, their reading. A key insight in this description comes from Baird's use of Hacking's definition of measurement in which the main function of a measurement if to produce a "stable numerical phenomenon" in a setting of rigorous control. Computer simulations, in fact, are the kinds of technical artifact that can and do produce numerical phenomena. In fact, even if we take only the narrow definition of computer simulations, this is what computer simulations strictly do. Furthermore, as far as controlled situations, it just simply does not get any better than the abstract realm in which some philosophers take them to operate. If computer simulations are, for example, anything like implemented models as Herbert Simon (1969) suggests - machine-automations of mathematical relations - then they are the kinds of 
instruments that Baird alludes to. While a lot of work is being done by the first part of the description regarding the generation of a signal this can be easily interpreted to be exactly what the display in some computer simulations is doing. We can think of an instrument which upon detecting a certain signal reacts accordingly. We can also think of an instrument which only produces such a reaction when other indirect values are computed, such as the ones that Morrison describes in particle physics. These two kinds of instruments are different in one sense. They do not both interact with the phenomenon in an equally direct way. However, they are also similar in that a computation must take place, whether it be analogous or digital in order for the detection to occur. If so, the difference is one of degree and not of kind and computer simulations can indeed qualify as a version of the latter kind (Morrison, 2015). Computer simulations also have to carry out, reproduce, a set of procedural specifications every time they are meant to represent whatever they are simulating. In this more physical sense, computers are reproducing a certain state of affairs as they implement the specifications of their simulation model. As we saw above, one of the things that simulations do is to encapsulate, through their procedure, the testing of models (Lenhard, 2007). Once a procedural hierarchy has been established to run dynamic equations of a system, the computer simulation can in some way test whether these dynamics correspond to the phenomena that researchers are investigating. But computer simulations do not only encapsulate knowledge regarding the principled theoretical values and the direct experimental data, they also encapsulate the procedure by which to transform/manipulate the content. That is, they encapsulate experimental setting too (Barberousse, 2019). As such they are a hybrid instrument in Baird's terms. 
There are, of course, other ways of cataloguing the kinds of artifacts found in laboratories. As I will show, computer simulations also fail to simply fall under one category or another in these other taxonomies. This simply provides yet another explanation for the recurring intuition that computer simulations are something that is always neither here nor there, but rather in-between of our efforts to categorize them. The difference here however is that this in betweenness is no longer characterized as happening at the level of meta-methodical aspects of scientific inquiry. That is, the in-betweenness of computer simulations is not in between theoretical and experimental practices, but rather in between conventional categories of instruments and artifacts found within scientific inquiry. As suggested above, instruments are already something that is in between experimental and theoretical practice. Now, we turn to see where computer simulations may figure within this third element of inquiry.

For Harré (2003), for example, not every piece of equipment in laboratory equipment is an instrument. Models, for example, are an apparatus. While instruments have elements that are causally related to the world, an apparatus merely serves as a "working model of some part of the world" (2003, p.26). Hence, Harré directly distinguishes the apparatus from the instrument. He thinks that the working model guiding an inquiry - that is, the specifications of an experimental setting, for example - is more like an apparatus (p.26). Apparatus, for Harré, is "an arrangement of material stuff integrated into the material world in a number of different ways" (p.19) He reserves the word instrument for "that species of equipment which registers an effect of some state of the material environment, such as the thermometer" (ibid). While the word "apparatus", to him, refers to the kind of equipment that is a model of some "naturally occurring structure or process" (Harré, p.20) Harré is right in emphasizing a distinction between those kinds of 
instruments that detect a property or register an effect — say a sensor — and the work done by a model. However, as we saw above, models do fit into the taxonomy laid out by Baird (2004). In other words, models themselves are the kind of technical artifact that can be viewed as scientific instrument. Baird, however, emphasizes the role of physical models as exemplars of his view. Computer simulations happen to be hybrids in both views. They require a physical implementation in that they are simultaneously performative and representative in nature. In the case of Harré's distinction, they are also hybrid in that computer simulations are a species of equipment that is capable of modeling. As we saw, again, in section 3.3 computer simulations are not just equivalent to the model, they are not just the specifications, but they are the thing with which those specifications are implemented. In this sense, computer simulations are both the apparatus and the instrument in that they are at the same time "an arrangement of material stuff integrated into the material world in a number of different ways", a kind of equipment that is a model of some "naturally occurring structure or process", and also a "species of equipment which registers an effect of some state of the material environment". What registering here means can be a source of tension. However, we can flesh out the 'registering of' as merely enabling a researcher to capture dynamic changes that would have otherwise been unavailable. This suffices for the argument that they are hybrid, particularly since Harré uses the thermometer as an example. The thermometer as we saw above in Baird's account, is in a way like computer simulations in that it uses data inputs to represent the presence of a property in a system. Consider that a digital thermometer in which the multiple components are to be considered independently of one another. There is a set of components that enable 'detection', namely those in contact with the thing whose temperature we are measuring. In fact, there is but a single component that interacts with the phenomena in question, namely temperature. The rest of the 
thermometer functions in virtue of the data to determine that something was in fact 'detected'. In this sense, computer simulations are similarly detached from the components that gather the actual data in the world, but their functioning in processing the data in such a way as to make a property accessible is similar to that of the thermometer. In this way, we can accept Harré's distinction and still claim that a computer simulation is a hybrid in relation to it.

Yet another way of distinguishing between kinds of instruments is that of Michael Heidelberger (2003). Heidelberger distinguishes between two functions of instruments in scientific experimentation. According to him, they either fulfill a productive or a constructive function. When a scientific instrument is productive, it produces a phenomenon that doesn't not appear normally in everyday human experience. A constructive instrument, on the other hand, intervenes in the experimental subject in order to modify its behavior (2003 p.146). If we consider that computer simulations are capable of elucidating properties of a system that are not easily found in the world and manipulating data in ways that are not usually available in the world, we can construct this as meeting the first conditions. Much of astrophysics, and or particle physics would be unavailable to us otherwise. So, we can agree that computer have the capacity to manipulate data in such a way as to mimic behaviors of a system that are not easily found in the world. Granted, as we have seen in previous sections, whether this constitutes 'doing experiments' or not is a contentious manner. However, we can always fall back and appeal to Morrison's conception that at least sometimes, in some cases, the way we conduct experiments in physics is not so far removed from the way experiments are characterized by simulations processes. If so, then we can say that the manipulation of data in fact constitutes an both the production of phenomena not easily found in the world and an intervention that modifies its 
behavior. Yet again, computer simulations manage to claim their status as hybrids. While the details in each of these cases can be vastly expanded, what this chapter is poised to show is that, at the very least, computer simulations are the kind of instrument that does not fit easily into conventional categorizations of instruments in scientific inquiry. In this sense, computer simulations may indeed be a novel kind of instrument that requires its own epistemic assessment with regards to its status in scientific inquiry. If this is so, it is not because it is a sui generis kind of method, or a third branch of inquiry all on its own. Rather, it is because as an instrument it may indeed have genuinely novel properties that therefore pose genuinely novel epistemic challenges. As we will see, it may even be the case that computer simulations fail to be sanctioned the way other scientific instruments have been sanctioned through the history of sophisticated inquiry.

Computer simulation, in conclusion, are hybrid epistemic enhancers (Humphreys,2004) in that they help us transform one sort of information into another, they help us enhance existing capabilities and they allow insight into areas that we would not have access to otherwise; they are hybrid in that they are often both capable of simulating the processes by which an experiment is conducted (Barberousse, 2019) — they are, in Harré's words, an apparatus, “an arrangement of material stuff integrated into the material world in a number of different ways"-as well as an instrument "which registers an effect of some state of the material environment." (2003 p.19); and finally, computer simulations are also hybrid in that they are capable of being both productive and constructive instruments, in Heidelberger's terms. In other words, they are able to produce (simulate) a phenomenon in an environment that does not exist in nature as well as of modifying the (simulated) behavior of a system through intervention. 


\subsection{Concluding remarks on computer simulations as instruments}

In this chapter I have outlined a series of steps towards a framework that establishes computer simulations as instruments. In particular I have argued that their distinct essential functions and particular features are those of technical artefacts of the kind we see designed, developed and deployed in science. This discussion also led to a comparison of computer simulations to other instruments and to a focus on their hybrid nature regarding several existing taxonomies of scientific artifacts. Computer simulations have been in use for a little over a half a century. Scientific instruments in general, such as the telescope and the norms that govern their use and inclusion in scientific inquiry, have also not been in use for that long, maybe three to four centuries. This timeline is shorter if we limit ourselves to those artifacts associated with the rise of the precision instrument industry in European countries during the Victorian era. Therefore, understanding the role devices in general play in scientific inquiry is no trivial matter and the philosophical literature is exceptionally scarce. This makes the understanding of computer simulations as such the more challenging, but also the more pressing. It is my hope that this preliminary but thorough discussion brings both, computer simulations in particular and instruments in general, back on the radar of philosophers of science.

This chapter represents what I take to be the first steps towards a more comprehensive account of computer simulations as instruments and it provides, at the very least, the fundamental elements towards a novel understanding of computer simulations that is beyond the conventional dichotomy in the existing literature. It also provides the backbone of an argumentative structure 
that identifies the distinctive nature of computer simulations vis-à-vis other elements of scientific inquiry as well as vis-à-vis other instruments. From this, a more exhaustive inquiry can begin that further delineates the kinds of epistemic enhancers these artifacts are. For now, it is sufficient to establish that computer simulations are neither like their formal constituents nor like the empirical settings in which they are deployed and that they are rather like the myriad of devices that enrich the former in the context of inquiry and make the latter even possible. Within this category, one can now begin to inquire about their novelty, their epistemic import and the adequacy of the reasons we can muster to justify our trust in them. This is something I elaborate on in Chapter 5.

The 'practice' view of computer simulation which will be discussed in detail in chapter 4 takes some of the elements discussed above to suggest that the difficulties in placing computer simulations within existing categories are evidence of the inadequacy of the task. That is, the argument goes, the reason why it is hard to find where computer simulations belong is that they are not a thing. In this view, computer simulations are not models, they are also not an experiment, and, most particularly, they are not an artifact — as in a concrete computational infrastructure. In this view there is no cohesive set of practices, expertise, and/or even disciplines and fields - as exemplified by the different elements of a pipeline and the different emphasis each of those elements get from experts with diverse backgrounds — where the term 'computer simulations' denotes any unified concept (Resch, 2017), let alone a unified artifactual object. More importantly for the purposes of this dissertation, this position argues that because simulations are a heterogeneous practice - a medium, a way of thinking — with heterogeneous components and a heterogeneous community of experts behind them then it is misguided to try 
to understand them as an instrument. I will call this part of their position the argument from heterogeneity. As we will see in more detail below, this strategy risks excluding canonical exemplars from their 'instrument' status as well as ridding the term from any particular meaning.

In the following chapter I will show that the argumentative strategies focused on heterogeneity, though constituted by correct observations, are ultimately mistaken in their conclusion to exclude computer simulations from being understood as instruments. As briefly stated above, the argument from heterogeneity is such that on close inspection excludes nearly all technical artifacts from being understood as instruments. A peculiar result of this kind of argument is that it particularly excludes those instruments that have had a significant historical and methodological impact in the history of science. This is non-trivial implication. While the general argument of this dissertation is that computer simulations are best understood as instruments, the argument of the following chapter is that the reasons why some philosophers hesitate understanding computer simulations as instruments are not very good ones. 


\section{Computer simulation as practice}

In this chapter I present an overview the 'practice view' of computer simulations. I also present a series of arguments against it. This view, as I will argue, interprets computer simulation to be a practice term similar to engineering and/or medicine. In some particular cases, this view explicitly refutes the possibility that computer simulations can be understood as instruments, or understood through what Resch calls “tool metaphors' (Resch et al., 2017) ${ }^{81}$. In this chapter I reconstruct the arguments found in the work of Resch et al (2017), which are explicitly used against the interpretation of computer simulations as instruments. As I understand these arguments, they all appeal to the heterogeneity of practices, components and implications of computer simulation. This heterogeneity is understood to preclude any interpretation of them as instruments.

There are a few reasons why this view is of particular importance in this dissertation and its project. Here, I'll briefly mention two. The first reason is rather straightforward. As I will mention in the paragraph below, what I call the practice view has particular adherents that have explicitly targeted views that use any language related to artifacts in their understanding of computer simulations. This provides for a particular opportunity to address specific arguments

\footnotetext{
${ }^{81}$ I take Resch et al., here to mean that any conceptual framework that treats computer simulations as analogous (hence the term 'metaphor' )to tools or instruments is fundamentally misguided.
} 
that have been deployed against my view of computer simulations and any views similar to it. The second, and more important, reason is that this view represents a more recent development in the philosophy of computer simulations. As we saw in the introductory chapter, there is a division between a narrow and a broad understanding of computer simulations. The narrow view seeks to understand them as extensions of formal methods. The broad view sees them as belonging to a category closer to that of experiments and experimental practices. The practice view seeks to broaden the concept even further by suggesting that computer simulations are even closer to the category of things like engineering, or medicine. They are not an artifact, they are not an experiment, they are not models, so the argument goes. Rather, they are an amalgam of practices, expertise and communicative endeavors that are deployed in practical setting within scientific inquiry. They are, in other words, like science itself. This push towards a broadening of the concept, is a genuine attempt to include the many heterogeneous aspects associated with putting together a computer simulation. But it is also a genuine attempt at making sense of the many heterogeneous aspects that go into using a computer simulation. This call for a broader concept has its origins in interesting work from the sociology of computer simulations (Saam, 2017), which brings together opinions, norms and processes from practitioners. However, it is from this same fact that the practice view also draws its weakest points. As I will argue in the paragraphs below, this sociological turn in the philosophy of computer simulations imported questionable inferences from the sociological descriptions from the field onto normative aspects of the epistemology of science. ${ }^{82}$

\footnotetext{
${ }^{82}$ In a sense, this sociological turn has its origins in philosophical work that sought to account for the social and political forces behind scientific practices in general. Kuhn is paradigmatic representative of these sensitivities I the
} 
As the most recent development in the philosophy of computer simulations, I take this sociological turn to be an important view to address. This is particularly the case since some of the reasoning behind this view has direct implications for the view that I am here defending.

Consider, for example, that in their introduction to the edited volume "The Science and Art of Simulation" Michael Resch, Andreas Kaminski and Petra Gehring write that computer simulation "is not at all just a new device, an instrument, but rather a medium-a dispositif, in which thinking, decision-making, and understanding itself are shaped.” (Resch et al., 2017 p.2) According to them, because the practice of computer simulation "changes (and will increasingly change) the way political decisions are made, uncertainty is handled, and the future is understood" understanding them as instruments, or using any tool-related metaphors to shed light onto their nature and epistemic status in scientific inquiry is therefore misleading. (2017 p.2) According to Resch, for example, simulation is a close relative of thinking (Resch, 2013 p.234), while for Gehring the way in which we sanction the practice of simulation is a makeshift consensus amongst practitioners who are in turn concerned with a good-enough solution to a specific engineering task. For Hubig and Kaminski (2017), this descriptive account of computer simulations calls for a pragmatic epistemology that forgoes a grand narrative of a model's accuracy in terms of its proximity to a truth about the state of affairs of a target system. They offer instead success terms linked to engineering approximations, 'truths' that are measured 
depending on whether a practical task is accomplished or not and not a truth that speaks of the reference of the simulation to the way the world is.

As we will see below, this position has important implications for the formulation of a satisfactory epistemology of simulation. In particular, by continuing to push a purely pragmatic embrace of descriptive elements of the practice, positions such as these stand in the way of a stronger normative epistemology of simulation. Specifically, it fails to provide an epistemology that takes instruments seriously. But even if instruments were not taken into account in the epistemology of scientific inquiry, as I will explain below, a pragmatic view of the role of simulation which merely enumerates the occasions in which practitioners feel satisfied with a local development in the deployment of a device would still be ineffective in capturing the deeper motivations of scientific inquiry ${ }^{83}$ By taking sociological accounts of the way in which practitioners come to terms with their work as 'good-enough', pragmatic accounts of epistemology of simulation risk to delegate the job of normatively evaluating a scientific methodology to existing practices. If it is true, as some of these views suggest, that sanctioning of computer simulation happens haphazardly, that practitioners' criteria are makeshift, and that trust emerges through epistemic entitlements, then the more reason why philosophers ought to be worried about the status of computer simulations in inquiry. If anything, the sociological description of the way in which simulations are now being developed, deployed and employed

\footnotetext{
${ }^{83}$ Pragmatic views must explain why science is distinct from engineering, invention, or industry, for example. Or why explanation and not just prediction matters when it comes to the understanding of a phenomena in a scientific context.
} 
constitutes reason to reject their inclusion in the laboratory as sound scientific practice. I explore this latter issue in the final sections of this dissertation.

In the following paragraphs I will provide an overview of the main argumentative elements that have recently led some philosophers to focus predominantly on these social and practical aspects of computer simulations. I call this the "practice" view of computer simulations. It is marked by a sociological turn in their description and analysis. Although these views can be found elsewhere (Barberousse, 2019), the best and most recent example of this sociological shift can be found on the latest compilation of works entitled The Science and Art of Computer Simulation (Resch., et al, 2017). In this volume of edited works and elsewhere in independent publications, philosophers of computer simulations like Hubig and Kaminski (2017 p.121), Resch, (2017 p.23), Gehring (2017 p.9), present a series of arguments that display some of the details underlying their position against understanding computer simulations as instruments. They point, for example, to the scientific and social ubiquity—-the distributed features—of computer simulations, the practical complexity regarding their sanctioning and their multifaceted implications as central features for the characterization of computer simulations. They also point to the deep epistemic implications that the practice is having and will have on the way we think and assess what understanding is in general. That is, computer simulations have the power to redefine the criteria by which we judge scientific evidence to be epistemologically satisfactory. In this sense, what we think we know, or understand, the benchmarks by which we judge such understanding to occur, will be redefined. The practice of computer simulations, they suggest, also has the power to provide novel ways to gain scientific insight, often through visualization but also through their capacity to render complex computations into humanly intelligible 
modeling dynamics. The inference here, according to Resch et al. (2017), is that because of these complexity by which computer simulations come about and because of the reach and scope of those parts of inquiry that are affected by their use, computer simulations cannot be understood as instruments; the telescope, the large hadron collider, the synchrotron — amongst othersnotwithstanding. This conclusion, as I will show, simply does not follow.

Nevertheless, together with sociological analysis of computer simulation practices (Saam, 2017) as well as insight from social (Gehring, 2017) and pragmatic epistemology (Hubig and Kaminski, 2017), the views presented above, are sometimes taken to be reasons to reject an understanding of computer simulations as instruments. The gist of their arguments is the following. Computer simulations are a set of heterogeneous practices and motley processes (Winsberg, 2010) whose epistemic import and validity can only be approached through a pragmatic — and often makeshift— framework similar to that of engineering or any other "craft put into action by practitioners." (Resch et al., 2017) In other words, in this view computer simulation is a practice.

The argument, or series of arguments — which will be more thoroughly sketched below—roughly consist of the following reasoning. Computer simulations, according to most philosophers of simulation (Winsberg, 2010; Gehring, 2017; Saam, 2017; Durán, 2018) are the kind of heterogeneous inquiry practice whose conception, construction and use require a vast network of assorted communities, expertise and components. These communities, components and practices are also heterogeneous in that different simulations will rely on different communities, components, etc. Because of this, there isn't even yet a cohesive theory of computer simulation 
(Resch, 2017), or a science of computer simulation (Gehring, 2017). More importantly, according to these views, because of the heterogeneous and disparate constitution of computer simulations mentioned above, there isn't an instrument to point to (Resch, Gehring and Kaminski, 2017).

While all of these observations about the practice, development and use of computer simulations may indeed be true, the inference from them to the conclusion that these are satisfactory reasons to exclude computer simulations from being understood as instruments is not immediately obvious. As I will show in this section, this line of reasoning is unwarranted. First, because instruments made of instruments exist; second, instruments that are developed, deployed and maintained by seemingly unrelated communities of experts exist; and third because instruments that have the capacity to alter the way in which a science is conducted and even to change what acceptable scientific criteria are exist. ${ }^{84}$ Of course, what this shows is not exactly that because of this then computer simulations must/can be instruments. For all we know, we have just been applying the term wrongly all this time. What it does show is that if this is the case, then we have been so wildly wrong that even what we took to be the most paradigmatic instances of the term

\footnotetext{
${ }^{84}$ An interesting thing happens here with computer simulations and the pragmatic approach to their epistemology: the pragmatic epistemologist (Hubig and Kaminski, 2017) as well as the sociologist of computer simulations (Gehring, 2017; Saam, 2017) aims to reduce the normative project of sanctioning computer simulations into existing practices in order to accommodate and account for the realities of the field (Beisbart, 2017; Barberousse and Vorms, 2014). However, the relationship between other instruments, such as the telescope, and the normative criteria by which their deployment was rather to make the existing criteria more severe and demanding than what is was (Van Helden, 1994). In other words, while pragmatist seek to bring normative criteria associated with computer simulations down to accommodate practice, other instruments brought the normative criteria by which an instrument was judged up to accommodate desirable/higher epistemic standards (Lange, 2003 p.123).
} 
track nothing. Telescopes may not be instruments. No composite technical artifact—an artifact that is constituted by other technical artifacts - may be an instrument under this view. That may be so. However, I take this to be an undesirable consequence of any view that tasks itself with understanding any part of the way science is practiced.

Despite these objections, which I will come to later on in this dissertation, it is important to elucidate the basic framework of the 'anti-instrument' positions in order to understand why they represent a recurrent challenge to the understanding of computer simulations as anything remotely similar to instruments. ${ }^{85}$ In this dissertation I identify a series of conceptual hurdles to the understanding of computer simulations as instruments. In this section I address several of them, which directly address the existing literature: the conceptual pipeline; and the arguments from heterogeneity, understanding and reach.

\subsection{The pipeline}

As mentioned above, there are several conceptual obstacles to understanding computer simulations as instruments that are taken by some-particularly Resch et al. (2017) but also indirectly by others (Hubig and Kaminski, 2017; Barberousse, 2019)—as evidence of the

\footnotetext{
${ }^{85}$ As I showed in section 3.2 and 3.3, some fairly recent work does admit the use of the term 'device', or 'tool' to refer to computer simulations (Barberousse, 2019) and some others hint at the term as a vaguely encompassing metaphor (Parker, 2009; Winsberg, 2010; Morrison, 2015). However, as Resch et al. point out in their objection to the use of these terms, these instances of the notion are often indeed metaphorical. As I will make clear in this dissertation, I am not using the term instrument as a metaphorical marker. Rather, as will argue, computer simulations are instruments in the fullest sense of the term.
} 
inadequacy of the instrument view of computer simulations. Part of the argument, as briefly sketched above, includes the fact that computer simulations are the product of a multilayered, multilevel, multicomponent, heterogeneous process. Often, this multilayered, multilevel, heterogeneous view of computer simulations as a practice is exemplified through the means of a pipeline and the pipeline is taken as evidence that computer simulation is a practice rather than a device. As we will see, when it comes to the pipeline depicted below, Winsberg (fig 1 right), for example, positions simulation as an activity for scientists to engage in (2010 p.58). By extension, computer simulations, for Winsberg, are only a computerized version of this activity.

The pipeline is a conventional depiction in the literature of computer simulations of the stages followed by practitioners from a target phenomenon to the results of a computer simulation (Winsberg, 2010; Resch, 2013; 2017; Szabó, 2015). Though there are many different versions of these pipelines in the practitioner's world, it can be useful to closely look at and compare a couple of these depictions from the philosophical literature as they describe the process in a more general way. The following is a depiction of the pipeline of computer simulations as depicted by Resch (2013), Resch et al. (2017) (fig.1 left) and Winsberg (2010) (fig.1, right): 

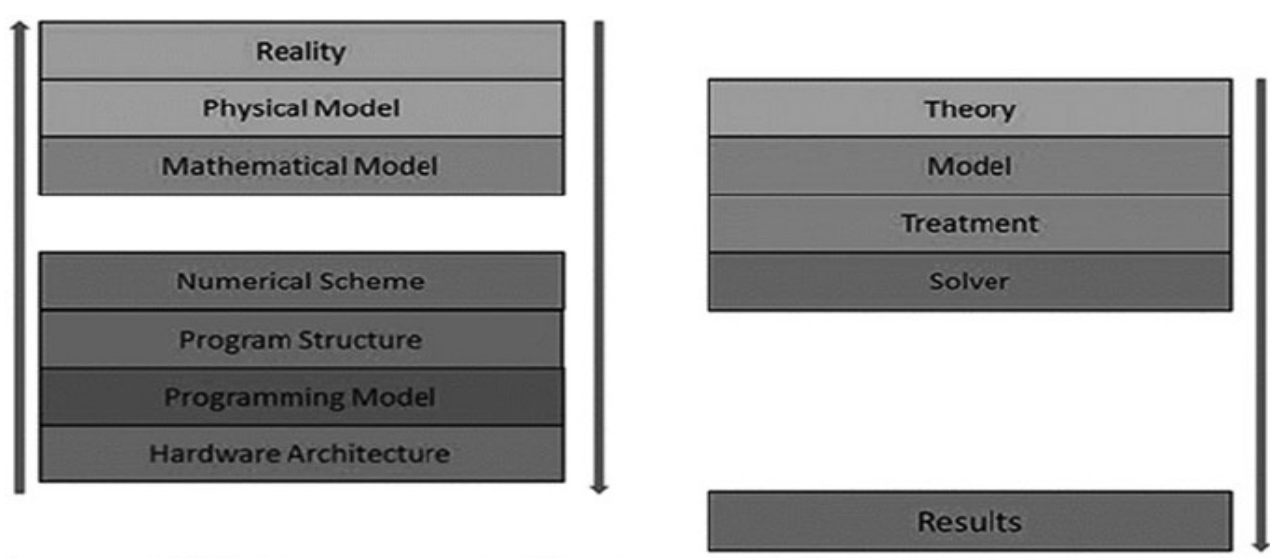

Figure 1 Two different simulation pipelines (Resch, 2013; 2017 (left); Winsberg, 2010 (right))

This sort of illustration of a simulation pipeline is deployed as a heuristic device - that is, an exploratory, often idealized, representation of a process - that elucidates the relevant components of a computer simulation and their place in scientific inquiry. ${ }^{86}$

It is worth looking at each of these steps in brief detail as these depictions represent a conceptual starting point for those who advocate understanding computer simulations as a practice (Gehring, 2017) and also those that think that they can't be understood as instruments (Resch et al., 2017). In the following paragraphs I begin by assessing the several stages of the simulation pipeline as depicted by Resch (2013) and then I contrast it with that of Winsberg (2010). Finally, I argue that the emphasis of both depictions and their accompanying explanations by the authors on the

\footnotetext{
${ }^{86}$ As you may note and as I will show in the following paragraphs, these pipelines are rather a confusing abstraction since they fail to identify where the computer simulation begins and ends. Instead, computer simulation is treated as a rather diffused entity present at all stages of otherwise conventional - that is non-computationally intensiveinquiry, i.e. at the point when a scientists looks at reality, at the point where she theorizes, at the point where results are scrutinized, etc.
} 
multiplicity of steps related to the creation of a computer contributes to a misguided conceptualization of computer simulations as an activity. This, therefore, serves as a false foundation to the idea that a computer simulation is the same as all the activities related to simulating.

In a 'simulation pipeline' (Winsberg, 2010; Resch, 2013; 2017) the process of developing a computer simulation is depicted as a series of modular items that goes from an inquiry into the world to getting results. In the case of the pipeline depicted in Resch's (2013; 2017) work (fig 1. left) the practitioner, or scientist, begins by looking at the world. This corresponds to the section called 'reality'. In the case of physical phenomena — to which Resch is purposely limiting his example to - a depiction of the physical interactions is conceived: an account of the entities, forces, and effects at play in the target system is delineated and a physical model results. ${ }^{87}$ From this depiction of physical dynamics (forces, mechanisms, etc.) a set of equations is developed that describes in a formal symbolic form the relevant interactions within the physical model. This is the called the mathematical model by Resch and can be seen depicted as the third stage pictured above (fig.1 left).

What follows the mathematical model, according to Resch's depiction of the pipeline above, is a series of transformations of the equations in the mathematical model into discrete steps that make them apt for a binary machine to provide approximate solutions: this is called by Resch a

\footnotetext{
87 Resch is here assuming a purely mechanistic account of scientific representation of the kind advocated by Craver.
} This can be problematic in many ways, but it is a problem beyond the scope of this project. 
numerical scheme (pictured as the fourth stage above, fig 1. Left) — a series of mathematical procedures that transform continuous equations into many discrete operations, as explained in section 2.2.1. According to the pipeline depicted above, and Resch's version in particular, the discrete mathematical procedures of a numerical scheme have to be later specified as logical procedures. That is, sometimes through the use of preestablished theorems that justify some of the continuous-to-discrete transformations, the steps established in a numerical scheme have to be detailed as machine-readable steps that become the program structure of the simulation to be implemented. Following, Resch's depiction of a simulation pipeline above, a programming model consists on mapping the program structure on to the hardware architecture-a specific machine, or set of machines as we saw in the description of supercomputers above depicted as the final stage of the simulation pipeline above, on the left side - which the process will use to carry out these procedures. ${ }^{88}$

Each of these elements is indeed relevant to an overall assessment of the place computer simulations occupy in scientific inquiry ${ }^{89}$ It is true, for example that at some point before one arrives at a computer simulation one must, or at least should, be engaged with the reality of a phenomenon to be simulated. It is also the case that other preliminary steps must be in place such

\footnotetext{
${ }^{88}$ Resch is careful to note that sometimes the process begins by actually looking at the hardware architecture. That is, the kind of machine(s) that one has available and then working one's way back through the pipeline to figure out the kinds of problems one can solve with it. He is skeptic of this process and even offers commentary on large investment in supercomputing architecture prior to inquiry parameters (Resch, 2013 p.240).

${ }^{89}$ Barring, of course, anti-realist instrumentalist positions that posit that any sense of reality we may have about a phenomenon is in fact a product of the artifact/perceptual and conceptual means by which we observe it in the first place (Hacking, 1983). In this case then there is no such instance/stage in which one "looks at reality and then $[\mathrm{X}, \mathrm{YZ}]$ happens after".
} 
as defining the parameters of the inquiry, narrowing the focus of our inquiry, knowing the available hardware with which we can run the specifications that will guide the program that supports the simulation (see footnote 92 below). It is true that these steps are important and, at least some, must be in place prior to the possibility of a computer simulation to function as it should. However, none of them is the computer simulation. In fact, only some of them are part of a computer simulation. Engaging with reality, for example, the first stage in Resch's pipeline, is as much part of a computer simulation as it is of any other empirical knowledge-acquiring practice. That we do so is not really a part of a computer simulation.

The point here is that, ultimately, the pipeline is depicting a procedure of production and not a product. How a product is made and what the product is and/or does are not the same thing. That is, the pipeline doesn't really say much about what a computer simulation is, rather it depicts the stages that those involved in the production of a simulation have to take into consideration. But it also betrays the assumption, as opposed to the analysis, that computer simulations are a practice, a method and not an instrument. For Resch, the main aspects of a simulation are on the programing and the hardware aspects. However, he also points to the pipeline depicted above and even appeals to the diverse emphasis that others have given to the other elements as evidence for the difficulty of developing a unified understanding of computer simulations (Resch et al., 2017). That is, he uses the pipeline as evidence that computer simulations cannot be understood as a single unified product, and this is further used to challenge calls to see them as instruments, or to dismiss any "tool metaphors" referring to them (Resch et al., 2017 p.2). 
The idea of the pipeline is to show, in a graphic manner in the style of industrial project management charts, the elements and steps that constitute a computer simulation. However, the gist of the simulation pipeline above serves to illustrate that these are steps of a practice, an assembly line of procedures meant to capture a practice and as opposed to an instrument. This view of computer simulation as a practice, a verb, is further supported by some sociological accounts of the role of instruments and their functions. Marcovich and Shinn (2017), for example, identify three types of function in Nobel Prize physics instruments: detection, metrology and control. ${ }^{90}$ Instruments, according to Shinn and Marcovich can identify the presence of (a property, a force, etc.), they can measure (distances, space, temperature, intensities, etc.), or they can provide control conditions in an experimental setting. ${ }^{91}$ According to them, simulation is not a fourth function of any instrument (p.353). To them, simulation "does not constitute a differentiated instrument function" but it "operates transversely across" detection, metrology and control (2017 p.353).

This reasoning is supposed to be strongly informed by close interactions with practitioners and the processes they undergo in an effort to bridge the assumed gap between those that theorize about computer simulations and those that work closely in constructing them.

\footnotetext{
${ }^{90}$ While detection identifies a presence or a particle, force or phenomenon, metrology "includes matters of distance, space, form, weight, temperature, intensity..."; and finally, control is characterized as the "intention and capacity to obtain an anticipated outcome." (2017 p.354)

${ }^{91}$ To Marcovich and Shinn, control can be understood in general terms as "having an objective" and the means to "supervise the processes necessary to fulfill the objective." (Markovich and Shinn, 2017 p.354) But it can also include the capacity to produce a new phenomenon.
} 
With this discussion of the pipeline, my aim is to show one more of the many conceptual obstacles that often serve as the basis for the default position that whatever computer simulations are, they are not unified technical artifacts. While I do not think that the pipelines illustrated above constitute a proper argument against the instrument view of computer simulation, the fact is that, at least for some prominent figures in recent debates in the philosophy of computer simulation (Resch et al., 2017; Resch, 2017; Gehring, 2017, Gransche, 2017; Durán, 2018) the pipeline serves as readily available evidence of the difficulties in understanding computer simulations in the way I suggest. Exploring the details, the assumptions and the discourse associated with these depictions serves as a clarificatory exercise that will further illustrate the position taken by those that advocate for the 'practice view' of computer simulations, which we will cover in detail in the following section.

\subsection{Practice View vs. the Instruments View of Computer Simulations}

In this section I contrast the views discussed above, together called the 'practice view' of computer simulations, with my own, the 'instrument view' of computer simulations. I do this by providing a detailed restructuring and analysis of several arguments - the argument from heterogeneity in its several forms, the argument from understanding and the argument from depth and reach — often deployed by the advocates of the 'practice view' and pointing at their inability to ensure their conclusion: namely, that computer simulations cannot be understood as instruments. 
Although I do consider my view to be an entirely novel alternative to the views presented throughout this dissertation, I would like to begin this section by noting that there are indeed a few instances in which the philosophical literature on computer simulations alludes to the fact that computer simulations can be understood using terms similar to the concept of an instrument (See Barberousse, 2019; Morrison, 2015; Winsberg, 2019). Yet, often, these mentions are laden with a vagueness that includes any tool, method or process in inquiry. These references treat computer simulations as tools in the same way a mathematical equation is a tool, a hammer is a tool, or a spreadsheet is a tool. In a sense, viewed through these uses of the term, the term 'instrument' can be taken more like a metaphor, a placeholder (Gransche, 2017), than a metaphysical claim about the nature of the object as a technical artifact. Given this interpretation, some philosophers, (see particularly the work of Resch, Gehring and Kaminsky (2017) mentioned above and discussed in detail below) have begun to attack "instrumental" terms such as 'tool' as incapable of providing an adequate framework to understand computer simulations and their epistemic status in scientific inquiry.

As I have made clear throughout this dissertation, my own use of the term 'instrument' is not metaphorical. It also does not appeal to an indefinitely broader class of things that can simultaneously include formal methodologies and also things like hammers. Rather, my use of the term can be best understood as referencing the class of technical artifacts - complex physical objects designed with specified functions related to a practical task — that are developed and deployed in the aid of scientific inquiry. The only caveat here is that when it comes to computer simulations in particular, the term does not yet say much about their place and their status as fully sanctioned scientific instruments. This is a question that will be explored in the last chapter 
of this dissertation. To recap, in the previous chapter I argued that computer simulations are instruments. In the last chapter of this dissertation I will argue that they should be treated — that is sanctioned, vetted, and/or understood— by scientist as well as by philosophers as instruments too. Whether or not they qualify as scientific instruments is an independent question that is also addressed rather than assumed in the last chapter of this dissertation.

The idea that computer simulations must be understood as something more that can be captured by using a "tool" metaphor — as Resch et al. suggest - makes sense when computer simulations are understood from the historical and sociological perspective of the development and introduction of computer simulations into scientific inquiry. For example, it is true that there is no cohesive and unifying set of principles guiding the practice behind simulation methods. There is no structured area of research studying the multiple components that constitute a computer simulation. As Petra Gehring suggests, despite the fact that the term "simulation science" is encountered scattered around engineering literature (2017 p.10) there is no central discipline to bring together the many distinct fields of expertise required to put a computer simulation together. Simulation is, according to her, at worst just a placeholder term, and at best an ancillary science (Gehring, 2017 p.12). Others see simulation as a methodology that creates a simulation pipeline in which diverse expertise and practice is required at different stages (Resch, 2017). To simulate, in other words, is to go through this heterogeneous process to gain insight about a particular phenomenon or feature of an object. For Hubig and Kaminski simulation is "the aggregate of transfers of elements and their relation from one representation in another for the purpose of expansion, revision, and alteration of our theoretical and practical references to the world" (Hubig and Kaminski, 2017 p.124). Simulation for Hubig and Kaminski is a series of 
representational translations from one way of 'seeing' a phenomenon to another, i.e. from quantification of real-world phenomena to mathematical model to computational model to visualization dynamics. Simulation, to those in this side of the debate is but a practical process that includes seeing something in the world, reality, translating that into a physical model, then into a mathematical model, then into a discrete scheme, then coded into a program and finally set up into a machine that can display results. While these steps are, in a sense, true stages of the process of creating a simulation, their description says nothing of what a computer simulation actually is.

Beyond being mere calculators, computer simulations are acknowledged to be more sophisticated analytical process that can and do encompass statistical analysis and other forms of intricate modeling; however, this sophistication is not taken to be a product of their formal accuracy and success but rather an outcome of the complexity involved in their creation. Winsberg writes, "the numbers are only the first step in generating a model of the phenomenon $[\ldots]$ The process of generating a model of the phenomenon involves sorting through the pile of numbers, tying to integrate it with other sources of knowledge, and determining which, if any, of the features revealed in that pile of numbers are trustworthy and reliable." (Winsberg, 2010 p.19). This suggests, again, that while computer simulations include the processing of numbers, the real work regarding their validity as a scientific project lies elsewhere. Namely, it lies in their validation processes and practices.

According to the 'practice' view of computer simulations, the construction of each individual computer simulation is a makeshift endeavor. These processes and their unification in a 
computer simulation are, in the words of Gransche (2017), a mise-en-scene project in which these "differentiated communities that have already been formed in (sub-) disciplines and have specialized on selected aspects of simulation and simulation research" have not yet come together as a cohesive group of researchers (Gehring, 2017 p.18). Further, computer simulation — "understood as craft put into action by practitioners" (Resch et al., 2017 p.3)—also takes a heterogeneous community to pragmatically put together a supporting framework for each act of simulating. This makes it so that both the process of creating a simulation and the process of using and sanctioning the results of the simulation should be understood as a heterogeneous artistic skill (Resch et al., 2017): no two simulation projects are alike. The community of experts that put it together is a heterogeneous bunch and the communities of those that use them are diverse and unrelated. Even further, as exemplified in the description of supercomputing above, the fact that a computer simulation is made up of a plethora of assorted components with distinct functions each and that often each simulation project requires a distinct mapping onto the available hardware architecture, is taken by those that advocate the 'practice' view of computer simulation as an additional sign that there is no conceptual unit capable of capturing what computer simulation is and does.

\subsection{The argument from heterogeneity}

Taken together, the positions briefly discussed above can be taken as providing a compelling framework that makes understanding computer simulations as instruments a questionable endeavor. The first objection comes in the form of an argument that implies that because computer simulations are the product of a heterogeneous process, then they cannot be understood 
as an instrument. I call this the argument from heterogeneity. This argument has a few

formulations that emphasize different sources of heterogeneity and use them in order to reach the same conclusion: the product of a heterogeneous process and/or a heterogeneous product cannot be understood as an instrument. In this section I elaborate each variation of these arguments and critically assess their limitations. In particular, I shall argue that even if we accept the observed sociological and physical features that make computer simulations be the product of a heterogenous process or be itself a heterogeneous product - a product of an irreducible, motley set of components - neither is sufficient reason to conclude that they cannot be instruments or understood as instruments.

The gist of the general argument from heterogeneity is that so many diverse components, so many diverse communities and knowledge, and so many diverse procedures can't be instantiated in a single artifact. At the very least, all the emphasis on community practice, on practical assemblages of broad processes and procedural components, leaves little room to envision a single artifact to point to. In the following sections I outline the main elements of this approach to simulation and point to this lack of imagination as that: an unwarranted imaginative constraint due mainly to a saturated discourse, a limited conceptual legacy, and a significant blind spot regarding the history of scientific instruments starting from the telescope all the way to the large hadron collider. 


\subsubsection{Heterogeneity of components}

As explained above, the general argument from heterogeneity against understanding computer simulations as instruments comes from the idea that computer simulations require a heterogeneous composite of practices, expertise, and sanctioning community. According to many prominent views in the philosophical literature, computer simulation is a motley set of practices, procedures, and software and hardware components (Parker, 2009; Winsberg, 2010; 2019;

Durán, 2018; Barberousse et al, 2019). This heterogeneity is often cited as one of the many challenges in providing a satisfactory account of what computer simulations are, what they do and what role they play in scientific inquiry.

Though it would seem at first glance disingenuous to extend the discussion of heterogeneity to a discussion pertaining the heterogeneity of components—-because it is hardly controversial that many technical artifacts are constituted by other technical objects (Simoulin, 2017)—it may prove useful to address the possibility of someone arguing for this extension before we move on to more specific claims regarding the heterogeneity of expert communities, methods and practices in the next section. In particular, this first step is useful because though no clear and explicit argument for such a position figures in the literature, the fact that computer simulations are made of many components is often mentioned - almost casually, as if already understood that it adds insult to injury — when discussing the epistemic difficulties of assessing the status and role of computer simulations in inquiry (Lenhard and Winsberg, 2010 p.258; Winsberg, 2010 p.6; Parker, 2009; Gehring, 2017). But also, it is useful because it allows us to begin to see a 
general structure of the bigger and more explicit argument from heterogeneity that focuses on practices and communities and not just components.

In the pipeline sketched above, the distinct components particular to a computer simulation can be found in the section of the pipeline dealing with hardware architecture (for Resch fig1.left) and on the solver section (for Winsberg fig. 1 right). The hardware architecture is for Resch the stage of development in computer simulations in which the engineering details of the implementing machine come back to haunt the process. That is, the components and specification of the actual computers that will run the simulations must inform, at the very least, three of the stages prior: the programing structure, the programing model, and perhaps even the numerical scheme. ${ }^{92}$ For Winsberg, on the other hand, this part is only named the solver where heterogeneous mechanisms come into play either in the form of coding practices or in the form of unifying engineering tricks that respond to specific problems and hardware constraints (Lenhard and Winsberg, 2010; Winsberg, 2010 p.8; Grüne-Yanoff, 2017). This is because to Winsberg the solver section is not only where one may find the hardware architecture, but rather where a step-by-step programming takes place. The epistemic challenges of the several detached transformations in the pipeline, for Winsberg, all play out in this particular stage of the simulation process.

\footnotetext{
${ }^{92}$ As you may recall, the simulation pipeline described in section 4.1, the stages leading up to a computer simulation include the numerical scheme (the transformation of continuous mathematical components of a model onto discrete mathematics), a programming structure (the logical steps required to implement the numerical scheme on a machine), and a programming model (which maps the programming structure onto the hardware architecture).
} 
We can formulate the argument coming from the heterogeneity of components to be something like the following: even if we are to point to a series of physical artifacts constituting the implementation of a computer simulation, the fact that they are a series of artifacts - and that they are motley in nature (Winsberg, 2010; Gehring, 2017 p.10)—makes it so that there is no one single instrument to point to when we are talking about computer simulations (Resch, Gehring and Kaminski, 2017 p.2). Hence, the concept of a computer simulation does not refer to an instrument but is rather a "placeholder" term for a composite procedure which tangentially also includes an implementation stage on a hardware structure (Gehring, 2017 p.10).

It is true that computer simulations are not 'just one device'; it is true that computer simulations in fact come together only when a myriad of other instruments are synchronized through a detailed schema of which functions happen where and when; it is also true that computer simulations happen only when all this instruments are functioning together. However, one cannot infer because of these facts that computer simulations are not instruments. To begin with, many contemporary and sufficiently complex technical artifacts seldom are just one device. This is particularly the case with software-intensive instruments, which rely on computer hardware - a complex device constituted by many other devices. While there may be epistemic issues related to their complexity and accessibility ${ }^{93}$, their being complex does not preclude them from being

93 Technical opacity (Kaminski, 2016), the fact that the number of components of one system may prevent an epistemic agent from accessing the details of its working is one problem that arises with complex systems. The many-hands problem (Nissenbaum, 1994), in which it is difficult to assign responsibility for a product whose production involved many people, is another. 
identified as 'a device' or 'an instrument'. The heterogeneity of components in computer simulations is not a sufficient condition for such a claim.

Consider the following. Contemporary scientific instruments are often not a single invention. They carry with them a long history of previous instrumentation. While some instruments are given a heroic position in the development of a scientific field (Simoulin, 2017), their history in fact shows that their creation is often only "permitted and conditioned by a whole series of instruments already in existence" (2017 p.417). The point Simoulin is making here is that not only that these predecessor instruments are historically required for the new instrument to be conceived, but rather that these predecessor instruments are required as embedded components of the novel devices.

In fact, as Simondon (1958) notes about technical artifacts in general, "the specific evolution of technical objects occur neither in an absolutely continuous not completely discontinuous manner; it is made up of stages that are defined by the fact that they produce successive systems of coherence; between stages marking a structural re-organization there can be an evolution of a continuous kind; this is due to the progressive perfection of details resulting from experience and use." (Simondon, 1958) According to Simondon, as we can see, there is a heterogeneous collection of methodology, expertise and components in most technical artifacts. ${ }^{94}$

\footnotetext{
${ }^{94}$ This is not to say that this fact is without its own challenges. As we will see in future sections, this fact alonethat computational methods, such as simulations, are the product of a wide and diverse array of components and expertise, or even lack thereof - brings about many challenging dimensions to the epistemic status of computer simulations and other software-instruments (Nissenbaum, 1994; Winsberg, 2010; Simoulin, 2017). But what this
} 
Fox-Keller (2003) makes this same point more specifically about computer simulations the following way:

"What we have now come to see as the epistemological novelty of computer simulation in fact emerged only gradually — not as a consequence of the introduction of any single technique, but as the cumulative effect of an everexpanding and conspicuously malleable new technology" (Fox-Keller, 2003)

The general lesson here is that computer simulations can be singled out as individual instruments even if they are complex and include a motley set of components. This is in direct contrast to the argument above suggesting that the heterogeneity of computer simulation components was sufficient evidence to deem terms like 'instrument' to refer to them as misguided.

Consider as an example of the discussion above the synchrotron, an instrument to produce $\mathrm{x}$-rays and deploy them for specific tasks. While several instruments within the synchrotron do different things - i.e. a linear accelerator propels electrons, optical chamber directs beams, etc.- - the synchrotron itself works as an amplifier or "booster" (Simoulin, 2017 p.421). While the synchrotron is made of distinct instruments — an electron accelerator, multiple beamlines and detectors, amongst many others - the structure that houses them all and allows the multifaceted and multilayered functioning to provide a higher-level functionality is the instrument itself. 


\subsubsection{Heterogeneity of practices, methods and communities}

In the section above we saw an argument focused on the heterogeneity of components in computer simulations because it was central to the practice view of simulations reconstructed above. A more defensible argumentative element of the above-mentioned strategy and a more widely cited feature of computer simulations is the heterogeneity of the methods involved (Hubig and Kaminski, 2017 p.), the practices required (Lenhard and Winsberg, 2010; GrüneYanoff, 2017) and the communities putting them together (Gehring, 2017). It is, as I showed in Resch, Gehring and Kaminski's arguments in the chapter above, the most compelling reason to deny that computer simulations are instruments. In this section I show the limitations of these arguments in a similar manner to the section above. That is, as I work through the history of the advent of computational methods in science and I show that the heterogeneity behind computer simulations is just like those of other instruments before them. If this is the case then there is not much in the argument from heterogeneity to exclude computer simulations from being understood as instruments while accepting that other similarly heterogenous instruments meet the criteria for inclusion. In fact, one can even say that the epistemic heterogeneity-the fact that so many diverse disciplines and areas of expertise have been involved in the creation of computers has been rather an asset and not a hindrance for modern computation. Such heterogeneity does not lead us to think that the computer itself is not a unified technical artifact. Computer simulations, as a software-intensive instrument, are no exception. The fact that a diverse community of experts puts them together helps maintain a somewhat sober outlook on their limitations (Parnas, 1990). I will begin this section by providing a brief overview of the advent of computer simulations into scientific inquiry in order to showcase the relationship between formal methods related to computation and their indispensable implementation in 
hardware. This can further clarify my position and the emphasis I put on both the functional artifact and its role as an instrument—a technical artifact—in scientific inquiry. Furthermore, this brief overview will serve as a set up to understand that other technical artifacts have been the product of heterogeneous practices, methods and communities and nevertheless can still be understood as instruments in their own right.

Most historians would, correctly, point towards mid $20^{\text {th }}$ century's war effort as the time-period when computer simulations rose to prominence in contemporary scientific inquiry. However, computational methods first made their first appearances in the analysis of physical systems in the early part of the $20^{\text {th }}$ century. By the mid $20^{\text {th }}$ century these methods were brought in as analog instrumentation that helped solve differential equations for the war effort because of the speed with which they were able to perform rule-based procedures. Single equations could be solved with continuous variable values with the help of two and three-dimensional structures called cams. These were built in a way that could yield a continuous computation to guide the inclination of a canon on a moving boat. These structures were rotated and tracked with a follower bearing that would push and pull other structural components whose hierarchical architecture was of the outmost importance to the suitability of the process to simulate its intended target (Clymer, 1993 p.19). While these devices are a long way from current computational methods, it is clear that their several components worked together as a single instrument. And this is precisely what this brief historical description is meant to show. If this is the case, then again, the argument from heterogeneity fails to show why computer simulations are singled out from being understood as instruments while other technical artifacts with similar histories are not excluded. 
Electronic devices soon replaced these mechanical ones. By the mid-century electronic computers already managed large parts of naval fire-control systems (Von Neumann and Brucks, 1946; Metropolis, 1987). They could compute "in five minutes the complex mathematical problem requiring two trained operators working with electric desk calculators two and a half to three hours to complete by hand." (van de Velde, 1960) In particular, computers were designed to yield "a complete ballistic solution (integration of half a score of differential terms representing the interacting factors of angle of departure, velocity, mass, weather, and so on) in less than the time of flight of the projectile." $(1960$, p. 210) The main focus for these computational techniques, however, was not what we now know as simulations. Their focus was the automation of mathematical procedures such as solving equations that were then applied in the field (Ware, 1953). In so far as the focus of these techniques was to solve mathematical problems they were not yet simulating anything other than discrete steps to integrated calculations (Fox-Keller, 2003), however, once more it is evident from the start that several distinct devices were a part of them and that they could still be recognized as a single computational instrument.

The purpose of the first implementations was to solve for discrete states of continuous differential equations. A decade later, a second wave of implementation deployed such techniques in still more complex problems. Here again, we can see that computer simulations were, despite being the product of a vast and diverse enterprise with heterogeneous agents and goals could be identified as a single instrument. Later, as we shall see, computational devices and computer simulations acquired the ability to encompass several tasks. When this happened, they 
did not cease to be a single instrument, but rather more general ones: instruments with multiple uses, like so many others.

In fact, it wasn't until a combination of computational techniques and devices came together that the modern notion of computer simulation came to be. Some of these techniques came directly from those involved in both the creation of computer technologies and the mathematics underlying their processes. In particular, they came from Ulam's and Von Neumann's work (Symons, 2008). Ulam's and Von Neumann's (1966) research on cellular automata showed that discretizing states of a system and representing them in grid form allowed researchers to study the dynamic development of such system via a set of minimal rules. By identifying only some of the key behavioral/causal components of a system, some of its future values or states could be emulated on a surface. This grid in turn provided researchers with an unprecedented visual dimension that allowed the tracking of a system without the need for explicit computational analysis (Hu and Ru, 2003; Symons, 2008). ${ }^{95}$ That is, the researchers did not have to do the math to follow the transformation of some of the system's spatial or numeric values.

\footnotetext{
${ }^{95}$ The innovation here of course is not the use of the grid but rather the computational process, the implementing of a machine procedure that could calculate and represent iteratively the discrete changes of a system's behavior. The deployment of a grid to understand a phenomenon goes back to Cartesian efforts and influence towards precision and accuracy in scientific and aesthetic representation (Mumford, 1934).
} 


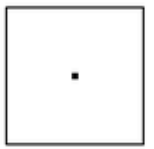

step 1

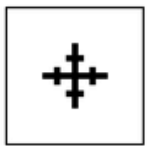

step 6

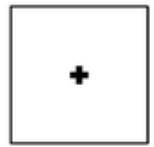

step 2

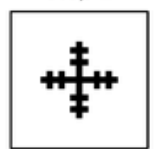

step 7

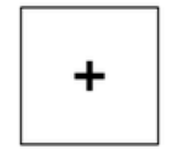

step 3

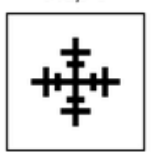

step 8

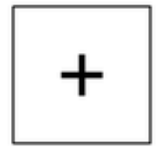

step 4

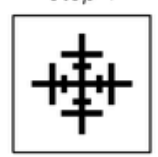

step 9

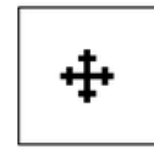

step 5

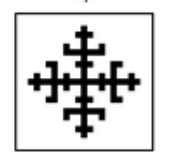

step 10

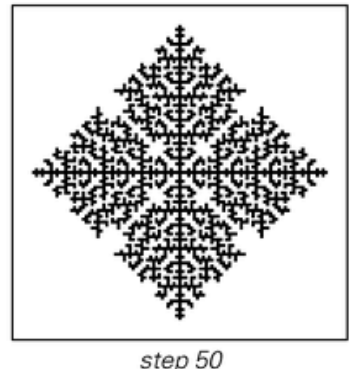

step 50

Figure 2 Iterated transformation in Ulam's 1967 procedures for 2D cellular automata (Wolfram, 2002)

Through iterations and statistical analysis of these iterations, these artificial systems began eliciting, or mimicking, consistently familiar behaviors (Dauxois, Peyrard, and Ruffo, 2005 p.4). ${ }^{96}$ At the time, however, this mimicry was still based purely on numbers. That is, the mimicry was established through the mathematical behavior of assigned quantities and values that were understood as equations assumed to model the dynamic relationships found in a realworld target phenomenon (Heims and Bailey, 1982). Simulations were only simulations of something insofar as the numbers in a system behaved like the numbers in a target system. What is important to note in the discussion in this last paragraph is that computational techniques are not merely thought of as abstractions. They are not like other mathematical procedures. When Ulam, Von Neumann, Wiener, and others devised these techniques they were simultaneously considering the machines that would have to be built for them.

${ }^{96}$ The visual aspect of computer simulations, and its early beginnings through cellular automata is a rich and complex topic. For someone like Simondon, for example, human understanding can be always reduced to visual understanding. For more contemporary philosophers of simulations, it is this visual aspect that uniquely identifies simulations. That simulations are only, and or mainly, a visual instrument is not exactly clear. 
In fact, these techniques were implementation schemes (Heims, 1980 p.139) ${ }^{97}$. This further supports the view, that at least initially, computer simulations were inseparable from the things that carried them out. In the following sections I will show that despite the level of abstraction involved in computational processes as complex as modern computer simulations, this fact - that they are instruments - hasn't changed that much. We can still single out an identifiable product, an actual artifact whose properties and deployment can be analyzed somewhat independently of the many components and expertise required for its production (Simon, 1996). That is, there are computer simulations in the world, they are things and they do things. To see this more clearly, we can go back to instruments such as the synchrotron mentioned above. The development and deployment of such an instrument requires a vast amount of people from different disciplines. This doesn't make them less of an independent artifact. This fact extends towards its use once in place. Even at specific, seemingly homogenous, stages of the process, such as when the accelerators are being operated, several small teams of technicians and engineers must be in constant communication and even collaborative compromise (Simoulin, 2017 p.442). Yet the fact that they are operating on a single functionally individuated artifact all at once remains. When it comes to operating it fully the synchrotron involves the input from specialists in magnets, geometers and engineers. This fact does not prevent us from calling it, using it and treating it as a single, unified, functional instrument. What is more important it does not prevent us from understanding it as an instrument. When questions arise about the reliability of the

\footnotetext{
${ }^{97}$ It is true that to Wiener, the focus of his novel insights was not to be on the physical constituents but on the behavioral patterns of machines and organisms (Heims, 1980 p.215). These patterns, however, were ultimately manifested in both his theoretical and technological writings as control and communication mechanisms, which depended on a physical implementation.
} 
instrument or the validity of its deployment for a given task we do not immediately resort to a sociological inquiry of its use, or to the theatrical aspects of its institutional praise. Rather there is an inquiry about the instrument's properties and functional characteristics as well as their relation (aptness) to the subject of inquiry. This inquiry into the means of inquiry is conducted by a wide variety of experts without diminishing the status of the device as a single, functionally unified instrument.

\subsubsection{The case of Galileo's instruments}

That many people, from diverse disciplines and expertise, are involved in the sanctioning of a complex instrument is not something new. Consider for instance the many extraordinary methodological steps that were taken by those trying to establish the epistemic superiority of Galileo's instruments - particularly both his most famous and also what some identify as (Koyré, 1957) the first scientific instrument ever. There are records that show, for example, that both Galileo's instruments and his novel controversial findings were not merely accepted because they were the best approximations at their time. Rather, a diverse community of developers, users and judges that included mathematicians, experts in light, crafters of glass and credible illustrators along with astronomers themselves were part of the development, sanction and deployment of the instrument. Unless we are willing, due to the involvement of this heterogeneous community, to demote the telescope from being an instrument to something more intangible, such as an experimental practice, then there is no reason why computer simulations should be excluded from being understood as an instrument either. 
When Galileo was trying to convince other scholars and the general public of the remarkable power of his instrument, he shipped some of his prototypes to persons of influence (Van Helden, 1994; 2003; Zik, 1999; 2001; King, 2003). The instrument itself and the maker's reputation were not enough by themselves to generate the trust required to include the device as a trustworthy technology in the canon of inquiry of the times. Galileo had to provide a method along with it. He provided a detailed manual of how to position the instrument, when the best viewing times were and even star maps to find locations in space more easily. Often, these instructions were provided with the aim of corroborating sightings that he himself was trying to convey (Van Helden, 1994; King, 1955). This method, however, did not always work. Some of the users of the instrument would be unable to work with the telescope itself, or would be unable to see the things that Galileo was trying to corroborate. This is in part why other independent methodologies had to be put in place by others, like Kepler and his associates in order to assert the authority of both the instrument and its designer. As we will see in the following paragraphs the communities surrounding these efforts were quite heterogeneous: they included statesman, mathematicians, philosophers, and even trusted merchants (Van Helden, 1994).

Kepler, particularly, took extraordinary steps to ensure what we would now call "objectivity" in corroborating Galileo's findings arrived at with Galileo's instruments. Importantly, these steps intrinsically $^{98}$ involved the inclusion of a heterogeneous community. Van Helden describes the

\footnotetext{
${ }^{98}$ It was part of their design and their function that they include a wide variety of notable characters form the scientific (intellectual) community as well as from the powers that be in order for there to be less (relatively speaking from an instance in which the people involved did not already enjoy a positive reputations) room for skepticism and hopefully bias. However, that they were already trustworthy characters I the community was only a
} 
recipe for certifying observations invented by Kepler the following way: "make the observatory a public space by enrolling fellow observers of high social status or other excellent credentials, have them draw what they see independently, and then compare results, thus confirming the observations by means of witnesses" (1994, p.13) Further, Van Helden notes "Kepler even went to far as to tell the reader of his published tract on the subject that Prague was his witness that these observations were not sent to Galileo although he owed him a reply." (Van Helden, 1994 p.13) Part of these procedures were described by Kepler himself as follows:

"We followed the procedure whereby what one observed he secretly drew on the wall with chalk, without its being seen by the other. Afterwards we passed together from one picture to the other to see if we agreed" (Kepler, 1611 as cited by Van Helden, 1994 p.12)

In order to ensure the credibility of both the instrument and its results, it wasn't enough to merely demonstrate its prowess. That is, simply using and testing the instrument with its appropriate instructions was not enough to sanction it as what we now know to be a scientifically acceptable device. Here we can see that Kepler took written note of his methodology for justificatory purposes, that he proclaimed independence as a third party free of conflict of interest and influence and that at the time of publication there had been no contact with biased sources (Van Helden, 1994). There was an explicit move to remove or reduce the possibility of human bias, unconscious intervention, and error by way of methods that could address as many counterfactuals the skeptic may come up with. This point, while useful in showing the presence 
and input of a heterogeneous community, will also be important when I talk about the epistemic superiority of scientific norms and practices and their inherent rigor.

Once again, this serves as counterfactual evidence towards the view that presence of epistemic heterogeneity in a sanctioning community of multidisciplinary experts is not enough to say that something is not an instrument. Just because it takes a community to produce and sanction an instrument it does not mean that the instrument is the community, or that the object of their sanctioning as well as the sanctioning norms by which the instrument is sanctioned are merely a community construct. ${ }^{99}$ This point is important because at least some (Gehring, 2017 p.12-13) in the literature on computer simulations appear to maintain this conflation. That is, they fail to recognize that an artifact may enjoy an independent epistemic life from that of the methods by which it was produced and/or the communities that produced it.

As I showed in chapter 3 particularly sections 3.1 and 3.1.1, computer simulations are independent artifacts with properties and epistemic challenges of their own. Hence, we should investigate them as such. For example, the challenges of sanctioning, vetting or trusting a computer simulation, are notably distinct from the challenges of sanctioning, vetting and trusting

\footnotetext{
${ }^{99}$ With regard to the norms by which an instrument is sanctioned: while some norms can and in fact are community constructs (preferences, paradigms, etc.), there can be instances in which an instrument is sanctioned by norms that are dictated by adherence to objective principles, this is particularly the case with functionally identifiable items such as instruments. Whether or not an instrument designed to detect a given property detects or does not detect said property, for example, is not a matter of community construct. Rather, it will be decided by whether the instrument in fact does or does not detect the property it was designed to detect. That it be somewhat accurate is not a social construct (though that it is preferably so may be one) but rather a preconditioned requirement of the thing itself with relation to the world with which it engages.
} 
the mathematical content they manipulate. As we will see in chapter 5 in detail, not only do mathematical equations have different properties from those of engineered artifacts, but our trust on them is the product of distinct epistemic warrants too: mathematics and other formal methods call for warrants for belief that are a priori, while technical artifacts such as computer simulations require warrants for trust that emerge a posteriori. If we go back a bit and think about the simulation pipeline in section 4.1 once more, the remaining task of my argument is to identify where the computer simulation actually is located within this pipeline and then talk about it exclusively as the instrument that it is. As discussed above, in the simulation pipeline, there was no computer simulation at the end of the process. Here I am suggesting that the computer simulation is the thing that was put together by all the previous stages depicted in the pipeline, and that it is this final product that is used by the scientist as an instrument to conduct inquiry.

It is worth noting as an aside that when the community of mathematicians and learned men mentioned above sanctioned telescopes, it was not merely due to consensus and pragmatic considerations. Rather, the processes by which they sanctioned the telescope were responsive to higher normative requirements: that there was consensus at all was due to the fact that the tests done on the instrument and the instrument itself were already better at satisfying a normative criterion: namely accuracy. In other words, not only did the telescopes work but they also did so in accordance to meta-methodological norms already in place that discarded or attempted to discard possible flawed criteria. This is evident, when — as I will describe below-tests that involved reading poetry at a distance were found to be easily gamed by participants who had memorized the poem. While instruments are a way in which scientists and scientific communities can confer a certain degree of authority — as is evident from the multiple 
demonstrative devices created by prominent men of science from Galileo to Newton-it is also the case that in some cases the authority precedes the instrumentation. This authority is not merely, as some suggest (Van Helden and Hatkins, 1994), the result of social and epistemic standing on individual members of sanctioning committee. Sometimes the sanctioning is the product of a methodology that in and of itself reflects superiority ${ }^{100}$ over conventional epistemic practices in everyday life (Zik, 1999). This point speaks to the importance of superior epistemic norms embedded in scientific inquiry and the sanctioning of its components. These epistemic norms are often in fact in tension with pragmatic concerns that call for the instrument to be deployed and understood as a series of localized empirical success that further its deployment. As I will argue in this dissertation, like telescopes, computer simulations should be subjected to these superior epistemic requirements and not merely accepted to be trustworthy devices on the basis of pragmatically driven adaptations of our criteria (Hubig and Kaminski, 2017) or nonevidential warrants such as epistemic entitlements (Beisbart, 2017; Barberousse and Vorms, 2014). Accordance with, and normative calls for meeting, enhanced criteria was the driving force behind the birth of the instruction manual printing industry that emerged along the precision instrument industry (Heilbron, 1993 p.10-13). This has also been the case for newer laboratory equipment (Baird, 2004, p.126). As we move to understand computer simulations as instruments, we must take into account this fact: whether or not something makes it as a scientific instrument has to do with whether or not it does what it is supposed to do in accordance with these rigorous

\footnotetext{
${ }^{100}$ By superiority I simply mean a level of epistemic rigor and/or epistemic hygiene that is meant to overcome some limitations inherent in conventional or everyday epistemic practice.
} 
norms and not just with whether or not it does what it is supposed to do. In other words, merely doing something, and or doing it well is not enough for science.

In this section I showed that a heterogeneous community was involved in the development and sanctioning of the telescope, which is a paradigmatic example of a scientific instrument if there ever was one. By doing this, I also showed that to exclude a device from being considered an instrument on the grounds that a heterogeneous community was involved would also exclude the telescope. Some may still want to push the argument and indeed strip the telescope of its status as a scientific instrument. Yet, in doing so they would be depriving almost everything that has ever been called an instrument of the same status. If this is so, then we need to ask the question: if technical artifacts such as telescopes are not instruments, then what is? Furthermore, they would also be questioning the very meaning of the term itself or even stripping it of any meaning it may have had.

In this dissertation I assume that there are things such as instruments-tools, devices, machines and other similar technical artifacts - in the world, that this term does have meaning (that it denotes certain functional objects in the world), and I argue from these assumptions that computer simulations are such things. More importantly, this section showed that instruments can and have had, from a very early age, a diverse community of arbiters and that they are no less an instrument because of it. 


\subsection{The Argument from Understanding}

In the previous sections, I analyzed a series of positions, which claimed that a computer simulation could not be deemed an instrument on the basis of the disunity of its components and the complexity of the sanctioning community processes required for their existence. If we were to visualize the arguments as positioned on a time-line in which computer simulations are at the center, these arguments would be to the left indicating challenges related to things that come prior to the computer simulations. That is, the aforementioned challenges are challenges that happen at the stages before the computer simulation is put together or as it is used. Another related claim that is exemplified in the second part of the quotation at the start of this chapter is that computer simulations should not be regarded as instruments because, as a novel addition to scientific inquiry and policy-making endeavors, they change the very way in which we understand "understanding itself" (Resch, Kaminski and Gehring, 2017 p.2). Resch et al. here mean two things by this. The first one is that computer simulations allow us to access the evolution of complex dynamics that would be otherwise inaccessible to our understanding, they make the results of some restrictively complicated calculations available to our otherwise limited epistemic methods. Hence, because of them, we can now understand more than what we could understand before. ${ }^{101}$ In this sense, our understanding has changed. The second way in which computer simulations change the way in which we understand 'understanding', according to this

${ }^{101}$ This is particularly the case if one takes a loosely pragmatic account of what 'understanding' entails. As we will see in following sections the opacity of software-intensive instruments does in fact challenge what we can say to understand. 
view, is that computer simulations can force us to reassess what we take as evidence. Computer simulations, in Resch et al.'s words, entail a 'transformation of the way in which science works in general" (Resch et al., 2017 p.1). This transformation as we saw in the section above, happens through methodology and community practices. But here, the authors - and other in the literature that make similar claims - are referring to a change in epistemology (Hubig and Kaminski, 2017; Primiero, 2019; Pias, 2011). Simulations, in short, change the criteria by which we say we have understanding. ${ }^{102}$ So, the argument is roughly the following. Computer simulations do two things that instruments do not: they expand our understanding towards uncharted territory and they modify the evidential criteria by which we conduct scientific inquiry itself. In the timeline considered above, these challenges are to the right of computer simulations. That is, the argument is a challenge to the 'instrument' view of computer simulations that derives from what takes place after a computer simulation is used. It is meant to show that simulations have an epistemic effect that goes beyond what any instrument is capable of. This is, once more, taken to be a reason why computer simulations cannot be referred to as mere instruments; according to advocates of this position they are activities, trading zones, techniques, mediums, etc. (Galison, 1996; Winsberg, 2010; Pias, 2011).

\footnotetext{
${ }^{102}$ In a more technical way, this camp also suggest that computer simulations change the way we understand understanding because they will move us away from conventional mathematical requirements of transparency and tractability that are associated with scientific understanding (Durán, 2018). I will say more about this in the concluding sections. Suffice it say here that in this respect I do believe computational methods in general are introducing a novel challenge to conventional epistemic norms in scientific inquiry. For reasons that will become clear later on what I do not agree with is in taking these challenges as evidence that such norms ought to be pragmatically weakened to accommodate opaque technology.
} 
In this section I will not address the topic as an issue in traditional epistemology. ${ }^{103}$ Rather, I will address it by taking note of what this assertion is intended to do. In particular, this claim is intended to support the view that computer simulations are not instruments by indirectly associating the kinds of developments described above as exclusive of other elements of scientific inquiry: practices, methods, etc. Here I will show that this is not the case. Instruments can and do change what we take as appropriate evidence, they do change the way in which we explore and understand the world. They also change the way we understand the world (Koyré, 1957). In short, instruments - and simulations in particular, as those in the 'practice' view of computer simulations agree — are capable of bringing about significant developments in the epistemology of science.

Judging from the citation at the beginning of this section in which any notion of "tool" is dismissed by Resch, Gehring and Kaminski (2017 p. 2) in their introduction to the "Art and Science of Simulation", they argue that instruments are simply not capable of moving inquiry and inquirers towards fundamental reassessment of our own epistemic capacities and the relationship of these capacities to the world. Though I have examined it in the sections above, here it may be useful to see the specific quote again, this time in full and with appropriate emphasis on the parts that I deem relevant to the current discussion:

${ }^{103}$ Epistemic concerns, particularly regarding warrants and novelty, will indeed be addressed in following sections. Here, however, I want to keep the discourse regarding scientific understanding at the level of history in order to show that the changes loosely alluded to by the authors in question have in fact happened in other instances due to the introduction of instruments. 
"Computer simulation changes (and will increasingly change) the way political decisions are made, uncertainty is handled, and the future is understood. Tool metaphors, therefore, are misleading. Computer simulation is not at all just a new device, an instrument, but rather a medium — and a dispositif — in which thinking, decision-making, and understanding itself are shaped" (Resch, Gehring and Kaminski, 2017 p. 2)

Taken together the argumentative core of the citation, once rearranged for clarity, is something like the following:

1) Computer simulation changes (and will change) three things:
a. the way political decisions are made
b. the way uncertainty is handled
c. and the way the future is understood

2) Computer simulation is rather a medium - and dispositif — in which the following are shaped:
a. Thinking
b. Decision-making
c. Understanding

3) Therefore, computer simulation is not at all just a new device or instrument. And tool metaphors [that refer to it as such] are misleading. 
The assertions in 1) can be assessed in a straightforward manner. First, computer simulations are indeed used to make policy-related decisions. ${ }^{104}$ This is something novel in decision-making procedures at the political level, simply because there were no computer simulations available prior to the advent of WWII. Secondly, going back all the way to the early days of the Monte Carlo method, computer simulations do allow a broader, faster and more thorough exploration of possible trajectories of a system. This in itself creates an approach towards possibility and therefore towards certainty — mentioned in their claim — that is different from pre-simulation times. It is so in a few ways: a large number unknown 'knowns' can be explored at higher speeds and lower resource cost, in a way that only automated model exploration can (Sugden, 2000); a large number of unknown unknowns can be brought to light through the exploratory methods of automated statistics in simulations; and, the ranges for optimal solutions can be visualized with lower risk of failure. Thirdly, if we accept the assumption that computer simulations yield insight about the development of different stages of a system in time, then a charitable interpretation allows us to see that — in terms of risk management and disaster anticipation, for examplecomputer simulation does give us a sense of peering into the future that we did not have before. Hence, we can see where the claim that computer simulations change 'the way in which the future is understood' comes from.

\footnotetext{
${ }^{104}$ For an optimistic account of the various ways in which this is justified see D. Scheer's (2017) work "Between Knowledge and Action: Conceptualizing Scientific Simulation and Policy Making" in The Science and Art of Simulation I: Exploring-Understanding-Knowing, edited by Resch, Kaminski and Gehring (2017).
} 
The items in 2) are a bit more difficult to assess, in particular because of the use of terms like 'medium' and 'dispositif'. These are terms from the discipline of Media Studies that are supposed to reflect a contrast between an artifact—external, hand-held, and individuated — and an immersive (conceptual, linguistic, epistemic) milieu within which we interpret the world and the knowledge we acquire of it (Pias, 2011). From this perspective simulations are closer to the latter than the former. While some of these concepts are important to understand the general framework behind some of the positions of those who like Kaminski and Gehring oppose the view of computer simulations as instruments, what is more important to grasp from this point of view is that these positions hold that profound effects into the way in which we understand the world are not possible by “just a new device.” (Resch et al., 2017) This is precisely what I aim to refute in this section of the dissertation. The argument form understanding is roughly of the same form as the arguments from heterogeneity, and just as I did with those, I intend to show through a historical counterexample that it does not hold.

First, let us try to understand a little more of the position exemplified by the quote above. Drawing from Claus Pias (2011) who in turn heavily relies on the historical analysis of computer simulations by Peter Galison (1996), Resch, Kaminski and Gehring (2017 p.2 fn. 4) accept the premise that reliance in computational methods such as the Monte Carlo method-itself a precursor to the modern computer simulation — ushered scientific inquiry into a "place paradoxically dislocated from the traditional reality that borrowed from both experimental and theoretical domains, bound these borrowings together, and used the resulting bricolage to create a marginalized netherland that was at once nowhere and everywhere on the usual methodological map." (Galison 1996) What this emphasizes—-beside the point elucidated in previous sections 
concerning the disparaged and heterogeneous nature of simulation research-is that computer simulations also changed what science is and how it is done (Resch et al, 2017 p.1). ${ }^{105}$ Peter Galison (1996) says, for example, that in the early days of computer simulations "in the process of negotiating the relationship of the Monte Carlo to traditional categories of experiment and theory the simulators both altered and helped define what it meant to be an experimenter or theorist" (1996 p.120). The result of which was that simulations constituted an epistemic trading zone (Galison, 1996), "an arena in which radically different activities could be locally, but not globally, coordinated” (1996 p.119). In this sense computer simulations disrupted not only the market of ideas itself but the nature of the currency by valuing a different set of skills (mathematical and computational literacy, modeling experience, etc.) and pairing them against those already in existence (theoretical depth, empirical intervention, field work, etc.). The point being that there was a deep disruption in the way science was conducted and the skills and criteria necessary to conduct it. Resch et al., Pias, and others, however, took this as evidence that computer simulations can't be instruments. ${ }^{106}$ As we can see from the deconstruction of their claims above, the elements in 3) are supposed to follow from 1) and 2). But this is a strange reason to disqualify computer simulations from being instruments, or to dismiss any 'analogy' to

\footnotetext{
${ }^{105}$ It also alludes, once more, to that in betweenness often ascribed to computer simulations which I explained (or explained away) in chapter 3.

${ }^{106}$ It is unclear that this is the case, particularly considering the discussion in chapter 4 . The way this ambiguity of computer simulations is being treated here follows the framework that is still trying to understand them in the context of a false dichotomy: either they are like theory or they are like experiment. I have shown this dichotomy to be limiting, misguided and unnecessary. However, for the sake of argument, I will not retake that discussion and will assume that what is being said of computer simulations is in fact the case as it is being said. I will then use this same argument to show the contradiction that follows when it is being used to exclude computer simulations from being understood as instruments.
} 
instruments as misguided, since many instruments, particularly those of historical importance and novelty, have done just that: they have revolutionized the way science is conducted and often have modified the way we understand what science is as well as what good science looks like.

Let us consider the telescope once more. When it comes to the practice of the science of astronomy the telescope brought about fundamental changes and it did so in at least three ways. First, the telescope changed what could be seen and how it could be seen just by virtue of the visual enhancement intrinsic to the instrument (King, 1955; Zik, 1999; 2001). Details about objects in the sky were now available at a higher resolution than ever before and previously unknown objects also began to be accessible to humans. Second, as we will see in the following paragraphs its advent also changed what was taken as proper evidence, as craftsman and men of science raced to certify ways in which to measure the strength of the newest - and supposedlymore trustworthy of the telescopes available on the market were. This change had at least a couple of dimensions to it. It changed what was taken as evidence for the findings purportedly accessible by the instrument, and it changed what was taken as evidence that the instrument itself was better fitted to access those findings. Yet, as I will detail below - and this is the third way in which the telescope brought fundamental changes to scientific understanding - the telescope also changed the way we looked at the world and our place within it (Koyré, 1958). For it changed astronomy as well as the perceived relationship between instruments, theory and observation.

Let us go through each one of these changes. First, Galileo was right in claiming that his early discoveries were significant not only because of the discoveries themselves but because of the 
instrument with which they were made. Here he is presenting his early work in his own words, as cited by Koyré (1957 p.88):

"In this little treatise I am presenting to all students of nature great things to observe and to consider. Great as much because of their intrinsic excellence as of their absolute novelty, and also on account of the instrument by the aid of which they have made themselves accessible to our sense"

As we can see, here Galileo is not only introducing a series of novel findings but a novel instrument that made them available. With the help of the perspicillum — an immediate predecessor of the telescope-Galileo was able to describe many novel facts about objects in space: a detailed account of the rugged surface of the moon, new planets, new stars, etc. More importantly, for the purposes in this section, besides all these new facts, in the words of Koyré, "there was also the description of an astonishing invention, that of an instrument - the first scientific instrument - the perspicillum, which made all these discoveries possible and enabled Galileo to transcend the limitations imposed by nature — or by God—on human senses and human knowledge" (Koyré, 1957 p.90). What Koyré is trying to emphasize in this rather colorful description is the fact that the telescope did indeed allowed, for the first time, people to look into what was thought of as 'the heavens' and beyond the reach of both our senses and our comprehension (King, 1955 p.37). It is clear that the perspicillum had changed not just what could be seen but also through which means it could be seen. This second point refers not only to the instrument itself, which after all was the only means by which such novel phenomena could be observed, but also to the fact that with the race to develop the instrument into an ever better 
one came a series of methodological norms that would forever change the way we assessed proper scientific practice (King, 1955; Van Helden, 1994; Zik, 1999; 2001). In other words, the telescope changed what we understood scientifically but also, as will become evident in the paragraph below, what we took to be scientific understanding.

When there is only one producer of a specific instrument and that instrument provides evident advantages not available before we may have no choice but to trust the instrument maker as an authoritative source. In other words, one may be entitled to trust the instrument and its maker for lack of an alternative. Galileo was also a practitioner of the science and had been making progress within it. Galileo, after all had access to his very expensive instrument the most. He could take his time and obsessively track elements in the sky. However, after the initial advent of his perspicillum, and later with the early days of his telescope Galileo began claiming things that were not so evident to others. The community of astronomers, mathematicians and intellectuals involved in the practice of astronomy required a certain amount of procedural and propositional knowledge about the instrument in order to use it accordingly. That is, they required a certain level of theoretical and mathematical understanding regarding the heavens and the objects therein but also some know-how regarding the ways in which to track them with his invention. And even then, just having those two things was often not enough to corroborate Galileo's findings. So, when he released his findings regarding Jupiter's moons - along with diagrams 107 _he led a serious methodological and informational campaign to convince others about their

107 The practice of releasing astronomical findings accompanied with a series of diagrams was in itself a novel technique only preceded by some accounts of comets. This alone reflects a new element in the way astronomy was 
validity. But even this proved to be insufficient (Van Helden, 1994). As described in the sections above, it was an independent committee led by the efforts of Kepler that through a challenging and novel set of methodologies established the credibility of Galileo's findings.

Furthermore, as the telescope became more widely available its incorporation into the canon of scientific practice — or the role it played in creating one — required a complete epistemic project that included developing and revising ever more sophisticated ways of discarding error, much like computer simulations ${ }^{108}$. Telescopes were tested with objects on earth and then tests of the tests were conducted. That is, as the instruments were tested so were the tests in order to ensure that they were measuring what they were supposed to be measuring (Van Helden, 1994) This also led to a change in methodology regarding error or bias correction (King, 1955; Zik, 2001). Letter tests, for example, the standard targets for early telescopes-like the ones you find at the eye doctor but placed miles apart—went through significant modifications. They changed many times over not just in content but in style and material too. Problems emerged early on when the tests contained fragments of literature that could be guessed by well-read segments of the population - the very same members of the population participating in having/or testing a telescope — after the first lines. Fonts too underwent changes due to tests on telescopes: fonts that

practiced hereafter. And while the instrument that Galileo was using enabled this new element of astronomical practice, it would not be correct to ascribe too much responsibility to the instrument itself for Galileo's decision to draw what he saw. Yet, the instrument Galileo was using did directly change the way observations from an instrument could be verified. It, in fact, instituted a method specifically designed to avoid possible human missteps.

${ }^{108}$ Computer simulations and many other software-intensive instruments require very different ways to deal with possible error than other simpler hardware devices (Parnas, 1990; Symons and Alvarado; 2016; Symons and Horner, 2019). 
made letters easily recognizable, even if not clearly seen, through the instrument were discarded (Van Helden, 1994 p.26; Zik, 2001). In short, the telescope changed quality control standards in the printing industry. Special paper, special ink, and even special ways of drying the letter tests were developed. We can see from this that that Galileo's influence and authority were not enough anymore to sanction his instruments and the findings acquired with them. They required not only a third person but also a whole body of properly vetted witnesses as well as a whole industry of assurances. This happened inside and outside of the community of practitioners close to the scientist. ${ }^{109}$ This is not just about practices anymore; it is about epistemology: reliability and objectivity as means for proper understanding were not instated before then as norms in scientific inquiry. In this sense, the practice behind using telescopes changed even what was considered as credible evidence but also the devices and norms we use to assess and ensure that credible evidence is indeed gathered. The telescope is an instrument if there ever was one. Hence, this is a direct challenge to the claim assumed above by those that dismiss the term 'instrument' to refer to computer simulations on the basis that instruments cannot have such an impact.

The argument presented by Resch, Kaminski and Gehring (2017) as well as those of Pias (2011) and Galison (1996) amount to stating that tool references are misguided because there is

${ }^{109}$ Galileo's findings regarding Jupiter were also corroborated in Rome albeit with some caveats. While the mathematicians in Rome had indeed ben able to corroborate the observations as described by Galileo, they were cautious to note that the interpretations of these observations was an independent matter. What this did was to sanction/certify Galileo's instrument as a mathematical instrument but not a philosophical, or what we now call empirical instrument (Van Helden, 1994; Price, 1980 p.79; Turner, 1993 p.21; King, 1955; Zik, 2001). I will speak more of this distinction in the sections below. 
evidence of grand-scheme changes in the ways we understand inquiry itself, changes to the way we understand our own position as inquirers and what can be known. Then the examples of the telescope and its precursor need to be reexamined. After all, history shows that they had such an impact. Here we can concede that computer simulations, when and if they ever get to be appropriately sanctioned and validated as proper scientific instruments (see Symons and Alvarado, 2019), can be said to have done just that. Galison, for example points out that the methods, practices and insights from those working on thermonuclear weapons introduced a novel way of doing science and in doing so "a debate grew about who might count as an experimenter or as a theoretician, and what would count as an experiment or theory" (1996 p.137). This happened in particular because of the use of computers to simulate physical processes. That practice changes because of practices - that our understanding changes because of practices - is not a revelatory claim. But the important part is that, as I have shown, there is no reason that the same cannot be said of instruments. Practices change because of the advent of instruments. Theories change because of the advent of instruments and criteria for theory and practice choice change because of instruments. There is nothing controversial in any of those statements. Therefore, merely citing this is not enough to disqualify understanding computer simulations as instruments. The views above would have two alternatives faced with this: either instrument such as the telescope did not have a bearing on changes of such kind, or they are not instruments after all.

As we saw above, one of the main claims of this camp is that in the absence of principled practice standards and homogenous epistemic methodology, those involved in creating a computer simulation have to gather a motley set of justificatory and pragmatic moves- 
interpretative artistry (Gehring, 2017) — as they move forward in a simulation project. This point is deployed to illustrate the ways in which these communities of practitioners and scholars pragmatically achieve a fundamentally transformative consensus on what is to be deemed as justificatory evidence to support their reliance and progress on a particular computer simulation. Hence a novel and unprecedented set of methodologies and practices emerges as the simulation moves forward. According to this view, this gradual process is transformative because the process of reaching consensus itself changes the very criteria by which evidence is deemed appropriate for the simulation task at hand. It was through the sanctioning of Galileo's results, and the work of others (Bedini, 1993 p.110) that the instrument itself began to be sanctioned. Instruments can and have in fact changed the way instruments themselves are sanctioned. ${ }^{110}$ Once more, we can see that the way in which the telescope was sanctioned, in particular, the way in which the evidential criteria and the practices of an engaged community changed with its advent, is not that different from what those advocating for the 'practice' view of computer simulations describe in relation to computer simulations.

\footnotetext{
${ }^{110} \mathrm{It}$ is important to note that there is a distinction between sanctioning the results of an instrument and sanctioning the instrument itself as an appropriate one to convey, or even arrive at such results. This is an important distinction that will later be useful in assessing the reasons why we should or should not immediately be trustful of computer simulations. We may, for example, say that a $2+2=4$ and sanction such a result on a priori warrants that appeal to the definition of the terms involved in the proposition, while at the same time wonder whether a toddler who arrived at the same result did so in an appropriate manner and then expanding on what an appropriate method is on the bases of processes justified through a posteriori evidence. Merely stating that something does the job does not elucidate the criteria by which we judge it to do it well or not.
} 


\subsection{The Argument from Depth and Reach}

The argument from Resch et al. analyzed above, specifically the contents of segments 2)— that computer simulation "is not at all just a new device, an instrument, but rather a medium — and a dispositif - in which thinking, decision-making, and understanding itself are shaped." (Resch et al., 2017 p.2) - is also an assertion about the depth and the reach that computer simulations have on the overall project of inquiry. That is, according to this view, the reach that computer simulation is unprecedented in the history of scientific inquiry. Accordingly, so the argument goes, so are the range and the depth of the implications of their ubiquitous influence across scientific and policy-making fields. Therefore, computer simulations should not/could not be understood as instrument, or so the argument seems to go. The implication being — to be found somewhere in a hidden premise, I suppose - that instruments, on their own, are incapable of being the agents of such deep and far reaching changes to the fabric of inquiry.

Yet, here too, the telescope meets, and even outdoes computer simulations by, those criteria. Koyré, for example is quick to point out that because of the advent of the perscipillum and later the telescope "one could even say that not only astronomy, but science as such, began, with Galileo's invention, a new phase of its development, the phase that we might call the instrumental one.” (Koyré, 1957) When the perspicillum came about, it allowed us to not only see things that had not been seen (Bedini, 1993) but also to think about things that had not been thought before and in ways in which had not been thought of before (Turner, 1993 p.21; Price, 1980). Hence, we can say that there is an instrument which enhanced our understanding in a deeper sense than merely enhancing our perceptual abilities. 
The resolution of telescopic observations allowed astronomers to understand cosmic distances in a way that had not been done before. allowed for a series of inferences concerning the nature of space and the things in it that was empirically but also conceptually novel. ${ }^{111}$ This was in part due to the fact that the telescope, or the perspicillum before it, was able to render some of the details of stars and galaxies visible. What is also interesting to note, particularly since the measuring of distance was of great importance for Galileo and his peers, is what the telescope could not do. The size of the stars seen though the perspicillum, unlike other objects on earth, did not seem to grow in size. This fact proved to be key in the arguments regarding their distances and therefore the distances in the heavens.

Although Koyré's assertion that the advent of the telescope changed cosmology itself is not seen as the final word on the actual extent of the changes brought about by such instrument, what is clear is that these changes were indeed of a depth and reach comparable to the depth and reach referred to above in Resch et al.'s argument. Furthermore, what this particular example shows is that changes with this degree of depth and reach were brought about by the advent of an instrument. Therefore, we can conclude that instruments, by themselves, are in fact capable of effecting such changes. If the argument from reach and depth is used to characterize the exclusion of computer simulations from the realm of instruments, we may have to give up on the term all together. After all, if a telescope is not an instrument, what is?

${ }^{111}$ See Ch. IV “Things Never Seen Before and Thoughts Never Thought” in Koyré, (1957) 
So far, the argumentative purpose of the sections above has been to respond to criticisms of the instruments view. The history of science in this context does not provide for a conclusive argument regarding computer simulations as instruments. However, what it does provide is a counterexample to the position above and which proves that instruments can be heterogeneously composed, that they can be the product of heterogeneous efforts on the part of heterogeneous communities and that they are not less of an instrument because of it. If this is so, then I have shown that none of the existing anti-instrument arguments surveyed here provide any principled nor practical reason to exclude an understanding of computer simulations as instruments. In particular, the argumentative focus has been to elucidate the underlying framework of those views that explicitly or implicitly suggest that the notion of instrument is at best a 'misguided' concept to understand computer simulations.

\subsection{Carving out simulations and making simulation instruments count}

While sociological descriptions of the conditions and practices of those involved in the creation of computer simulations are important, by themselves they are not adequate to capture the fact that the inquiry at hand is concerned rather with the end product of their efforts - an instrument, its specific properties, features and epistemic limitations — and not with the overall conditions that make scientific endeavors such as theirs possible. Furthermore, I point to the fact that strong reliance on descriptive accounts of what practitioners of computer simulations must undergo risks taking such accounts as if they were able to provide much sought-after normative content. As I will argue in detail in sections 5.3 and 5.4, this is not the case. 
As mentioned above, the main point of this section is to illustrate that while many different disciplines, components and expertise can be involved in the creation of a computer simulation there is a product at the end of the multidisciplinary pipeline - namely a computer simulation. Conventional views tend to include the theoretical and modeling efforts as well as social aspects between the parties involved (Schembera, 2017 p.54) as being part of the computer simulation. It is true that some social elements-such as political alliances, community consensus as well as economic constraints - have a bearing in the development and deployment of computer simulations in general. However, this is also true of most components of scientific inquiry and certainly true of the development of scientific instruments. Hence, these features can and should be counted as part of a sociological and other descriptive perspectives. ${ }^{112}$ However, when it comes to the particular epistemic analysis of an artifact - be it a computer simulation, a telescope or when assessing the epistemic merits of a specific method-these elements (the social, the political, the financial, and the makeshift engineering practices) can be broadly seen as the necessary preconditions of all scientific inquiry and thus not particularly informative as to the status of artifacts or methods. That is, if the social nature of scientific communities, the political aspect of resource allocation, and the non-formal elements of assembling something have to be considered for any scientific endeavor, then they must be considered for all scientific endeavors and therefore these aspects are not particularly informative about what is of interest regarding a specific instrument, or method, or practice within scientific inquiry. Rather, these are general

112 Kuhn famously advocated for the inclusion of social dynamics such as politics, biases and resource management in the analysis of the forces shaping scientific inquiry (Kuhn, 1969). 
concerns of science. Here, however, we are not trying to figure out the epistemic status of science, but rather what is the epistemic status, role and import of a particular instrument deployed in science. Scientific inquiry in general is developed and framed through social processes that include consensus-seeking practices and resource allocation considerations. To make these elements the focus of epistemological debates related to computer simulation risks missing the fact that a computer simulation is a thing in and of itself, an independent element of philosophical importance, namely a scientific instrument. In other words, focusing on the social and political elements of social practices of those involved in creating computer simulations misses the fact that what we are most interested in trying to explain is the phenomenon of computer simulation rather than anything else. Hence when we ask about the place and import of computer simulations, this end product, the computer simulation itself, whose epistemic role we are- or ought to be-investigating deserves philosophical attention independently from the broader social context in which it is developed.

As indicated above, understanding computer simulations as instruments is a significant departure from the mainline of philosophical discussion on this topic. ${ }^{113} 114$ As I will show below,

\footnotetext{
113 As we saw in section 3.2, making the case that computer simulations should be understood as experiments is quite a non-trivial task. An interesting point however, is that because of the difficulties in making the case for computer simulations as experiments, the conversation has once again splintered off and taken an even broader approach in which the experimental aspects of computer simulations and its heterogeneous components are taken to be part of a broader practice like medicine, or engineering. In this sense, when someone refers to computer simulations, they are referring to the whole practice of simulating rather than to a particular experiment or a device of any sort (Resch, et al., 2017).
}

My view is a response to the dichotomy between the narrow and the broad view of computer simulations as well as a response to this latter position.

114 The dichotomy between theory and experiment that has permeated much of philosophy of science has produced insights regarding the importance of theoretical principles in experimental practice in generating robust scientific results. It has also consolidated long-standing practices highlighting the experimental aspect of hypotheses-testing 
epistemological debates surrounding computer simulations have focused on the role of the communities involved (Gehring, 2017), the motley elements of multiple practices (Winsberg, 2010; 2015), and their experimental and experiment-enabling uses (Parker, 2009; Barberousse 2009). In particular, as mentioned previously, a large part of the contemporary debate on computer simulations has focused on whether computer simulations are like theoretical models or like experimental practice and not on the actual machinery, the integrated artifact, with which these practices and experiments are carried out. The argumentative burden of this dissertation is to show that viewing computer simulations as scientific instruments can shed new light on these debates and that my approach goes beyond both sociologically-oriented discussions and the models and experiments perspectives from philosophy of science. The principal contribution of the instruments view is its potential for shedding light on questions of reliability and trust. This dissertation argues in Chapter 5 that while computer simulations ought to be understood as scientific instruments, merely doing so does not serve to ground their epistemic status. Rather, like other instruments in science, computer simulations must be developed and deployed always accompanied and constrained by both strong theoretical principles and reliable empirical evidence.

central to constructing robust theoretical frameworks. Yet it has little to say regarding the importance of instruments in conducting said experiments or their role in establishing the credibility of theories. In many ways, as some historians of science have noted debates in epistemology of science, which have for the most part focused on this dichotomy between experiment and theory, have "tended to obscure the independent role of instruments in science." (Van Helden and Hankins, 1994 p.6) The debate surrounding the epistemic status of computer simulations is no exception. 
As I have showed in previous sections, existing debates in the epistemology of computer simulation have had little to say about the status of instruments as either bearers of knowledge or as enablers of knowledge independently of their relationship to either theory or experimental observation. One thing is clear from the previous decades of these debates on the status of computer simulations: understanding whether computer simulations are one thing or the another - that is, providing a concise answer to the question of "what is a computer simulation?'- is highly relevant to the assessment of their trustworthiness and hence also to the assessment of their merit as sanctioned components of scientific inquiry. As I will show at the end of this dissertation, to say definitively that computer simulations are instruments and that they ought to be understood and, more importantly, sanctioned or vetted as such, provides a new conceptual framework that reframes our approach to their epistemic status.

As noted in section 1.1, recent debates in the epistemology of computer simulation have sought to lessen the conceptual challenges that computer simulations face when compared to other practices and methods in scientific inquiry. Computer simulations are often compared to traditional empirical experiments and are regarded as an inferior source of observational material. Similarly, when compared to more formal uses of computation in inquiry, such as computer-assisted mathematical proofs, computer simulations are regarded as less rigorously developed (Barberousse and Vorms, 2014). And yet, their ubiquity and their evident success suggests that there are some reasons to, and some strategies by, which we can trust their deployment in science. To this end, philosophers and epistemologists of science have sought to develop strategies that suggest such instances and reasons while at the same time acknowledging their limitations vis-à-vis other elements of scientific inquiry. Some of these approaches towards 
an epistemology of computer simulation have focused on the kinds of truths that practitioners should concern themselves with and suggest that task-oriented successes are more pragmatic than broad theories of correspondence in scientific representation in computer simulation (Hubig and Kaminski, 2017). In other words, they suggest that unlike with other kinds of theoretical and scientific representation, what should concern us about computer simulations is not their ultimate correspondence to a Truth of the matter, but rather the little truths with which engineers and practitioners can cumulative build computer simulations and solve technical problems. Other approaches have sought to distance computer simulations from their formal interpretation by treating them as an art form (Gransche, 2017). This latter interpretation seeks to accommodate the view that computer simulations are of a motley (Winsberg, 2010) nature that, as a practice, is a heterogeneous emerging field without much formalization. Treating computer simulations as analogous to artistic practices, however, as Gransche (2017) does, ends up conflating a descriptive account of computer simulation practice and their epistemic environment (i.e. that simulations are often assembled with the help of ad hoc engineering practices and a motley multidisciplinary effort) with a normative epistemic account for adequate and sanctioned introduction of a novel element into scientific inquiry. That is, instruments deployed in science are not—and should not, as I will argue throughout this dissertation — accepted merely because they are introduced into scientific methodology as possible solutions to outstanding problems. If these pragmatic and sociological interpretations briefly mentioned above manage to capture some true elements of the way computer simulations are put together, it is unclear that they have what is necessary to construct a normative project about epistemic requirements for a new method, practice, or-more importantly—a device in science. 
Understanding computer simulations as instruments can help us ground epistemic questions that focus on the assessment of an artifact deployed in the service of science. That is, acknowledging the artifactual nature of computer simulations allows us to put them in a category with tangible precedent of what it takes to belong in scientific inquiry. When we wonder whether a given technical object—say a stick or a rock — is particularly fit for a specific task we do not ask whether the object is an art form (Gransche, 2017), or whether it is a social construct (Gehring, 2017), or whether its introduction calls for distributed and pluralistic epistemologies. What we ask is whether the object itself is capable of fulfilling the functions required by the task and/or whether it does so by meeting specific predetermined standards. It is not enough, for example, that a stick can stir a chemical solution. It should also be made of a material that can stir without dissolving itself, whether or not it can contaminate the process, and whether or not it is used in a manner that responds to superior norms of procedure. Rather, as technical objects to be used for a task - as instruments - the questions about the reliability of a stick or a rock for a specific task are about their material constitution as well as about their functional capabilities. The questions refer to the kind of thing they are, the kinds of things they can do and to whether or not we have proper epistemic reasons to use them for the specific tasks and contexts in which they are deployed. Rather than seeing them as a set of disparate and loosely practiced methods (Gehring, 2017), a medium or way of thinking (Resch et al., 2017), computer simulations should be assessed and sanctioned in the same way other instruments are. They are technical artifacts and as such they should be subjected to rigorous reliability tests, perhaps made in such a way that mirrors the precision instrument industry that emerged through the Victorian era. 
As I have shown in sections 4.3.3, 4.4 and 4.5, examples from the history of science can show that not every instrument deployed in the aid of science ought to be taken at face value. Rather, each of the instruments exemplified in those sections were subjected to numerous skeptical tests and rigorous reliability assessments that transcended — or were looking to transcend — not only the limitations of the instrument itself, but those of the community of scholars that was going to employ them and even the limitations of already established methods of testing. The way by which instruments provide solutions, that the solutions are in accordance with known theoretical principles, and that a community of practitioners understands the underlying processes by which these instruments arrive at the solutions is integral to their sanctioned inclusion into scientific practice. This is in sharp contrast to the suggestion that they should be accommodated by our epistemic requirements. The way things are done should not always be taken to be reasons to change the way we think they should be done. Computer simulations are rapidly expanding their presence across disciplines and projects, and rather than seeing them as a set of disparate and loosely practiced methods (Gehring, 2017), a medium or way of thinking (Resch et al., 2017) computer simulations should be assessed and sanctioned in the same way other instruments are.

It is important to note, at this point in the dissertation, that there is another obstacle to understanding computer simulations as instruments but that I cannot - for reasons of space, scope and depth — treat in this dissertation in detail. Nevertheless, this obstacle deserves to be emphasized, if only briefly, once more before I move on. The obstacle is that instruments per se have not been seriously investigated by philosophers and epistemologists — and, until fairly recently, historians_ — of science (Heilbron, 1993; Van Helden, 1994; Harré, 2003; Baird, 2004), with the exception of a few notable examples (Hacking, 1983; Humphreys, 2009b). While it is 
difficult to do justice to this characterization in such short space, it is reasonably uncontroversial to say that, for the most part, the epistemology, history and philosophy of science has been driven by discussions on the tensions between theory and empirical experimentation. Neither of which treats instruments as a serious, independent element of inquiry. Instruments, for the most part have been treated as either an extension of theoretical aspects of inquiry ${ }^{115}$ or as mere aids to experimental concepts (Baird, 2004). However, as I have shown in chapter 3, instruments do have this independent epistemic import in inquiry (Turner, 1993; Harré, 2003; Baird, 2003; 2004). And while this dissertation is mostly a treatment of computer simulations as instruments, it also serves a more general purpose of bringing the role and position of instruments in scientific inquiry to the fore for philosophers of science.

${ }^{115}$ Much has been written for example about the theory-ladeness of instruments and therefore of their inability to objectively claim direct access to the way the world is (Chalmers, 2003). 


\section{Implications: Computer Simulations and Epistemic Trust}

The arguments in the previous chapter sought to establish the distinct character of computer simulations as scientific instruments within the general landscape of scientific inquiry. One of the arguments of that chapter sought to establish that computer simulations are not distinct as a novel, sui generis, addition to the ontology of scientific methodology. They also do not represent a new science. They are distinct and novel, however, as part of an already established third branch of scientific inquiry: namely they are an addition to the set of instruments with which we conduct scientific inquiry. As such, computer simulations do involve challenging problems for the epistemology of science. Computer simulations call our attention to the more general need for a genuine attempt at an epistemology of instruments (Baird, 2004). While this general project is beyond the scope of the present project, there is clearly a need for more restricted analysis of the novel features of computer simulations relative to other technical artifacts

In this chapter I will argue that in our efforts to understand computer simulations as instruments some substantial changes follow to the way we can and/or must establish their epistemic status as devices capable of enhancing knowledge acquisition in scientific inquiry. Although I will elaborate on the details of the following three points in thorough detail in the sections below, for now, these changes can be briefly characterized the following way:

1. Computer simulations should not be considered purely formal methods that transparently covey mathematical or similarly formal information without substantially modifying such 
content: this includes the alteration ${ }^{116}$ of the epistemic warrants behind the content they manipulate. Accepting that the warrants supporting the kind of content computer simulations manipulate are distinct from the warrants that justify them as devices implies that we must reassess their epistemic import independently from the formal methods they are often associated with.

2. Because instruments such as computer simulations are not transparent conveyers, philosophical appeals to epistemic entitlements - a non-evidential epistemic warrant that operates under the assumption of the absence reasonable doubt — to justify our trust and reliance on them is an inadequate epistemic strategy. Furthermore, epistemic strategies that seek to pin the trust on the results of computer simulations to epistemic practices such as reliance on expert testimony are similarly inadequate.

3. And finally, as illustrated in previous chapters by the example featuring the many efforts to establish the scientific reliability of Galilean telescopes, having an epistemically satisfactory reason to accept a device as scientifically reliable requires more than a pragmatic reliance on the accuracy of their predictive power. Appeals to pragmatic justifications for computer simulations are inadequate. Treating computer simulations as

${ }^{116}$ As will be shown later, the term 'alteration' here is used in reference and contrast to Tyler Burge's notion of content preservation. Burge's notion frames content preservation as the ability of a method to transmit information without introducing, at any stage of transmission, any distinct justificatory content from the one that already underlies the content being transmitted. 
scientific instruments will also subject them to the same rigorous epistemic standards as other instruments in scientific inquiry.

\subsection{Epistemic entitlements and computer simulations}

There is a range of puzzling questions concerning the epistemological framework with which to assess the status of computer simulations in the context of scientific inquiry. This is particularly the case when views such as the ones discussed at the beginning of this dissertation are endorsed. When computers simulations are understood to be a completely novel practice or methodology, almost a new way of doing science (Rohrlich, 1991; Kaufmann and Smarr, 1993; Nieuwpoort, 1985 [as cited by Paul Humphreys, 2004]) justifying our sudden and ubiquitous reliance on them across scientific disciplines becomes problematic. On what grounds are we justified to trust the results of computer simulations? If they are a completely novel way of doing science, do we need a new epistemology? (Primiero, 2019) ${ }^{117}$ If this is not the case, if they are somewhat similar to other elements of scientific inquiry, what are the existing epistemic resources to assess their status in the laboratory? All these questions point towards a need for a justificatory source for our reliance in such devices. In epistemology these are questions about epistemic warrants. The sources of justificatory force explaining our reasons to trust. Since my response to some of these questions, as argued for in previous chapters, is that computer simulations are similar in

\footnotetext{
${ }^{117}$ For more details see Winsberg's (2019) updated entry on "Computer Simulations in Science" at the Stanford Encyclopedia of Philosophy.
} 
nature to - and should be sanctioned as — other instruments, then the question becomes clearer: what epistemic warrants back our reliance of computer simulations as scientific instruments?

A growing tendency in the philosophical literature on computer simulation (Barberousse and Vorms, 2014; Beisbart, 2017) is to appeal to a particular kind of epistemic warrant, called epistemic entitlement, as the kind of epistemic warrant that can provide such justification. An epistemic entitlement is a non-evidential epistemic right to hold a belief that something is the case just in case there are no reasons to doubt that it is indeed the case (Dretske, 2002). I may be entitled to believe, for example, that given certain circumstances (of limited time, depth and intricacy of subject matter, etc.,) — on grounds of good authority and without having to look any further for evidential support - that most of the content in my physics textbook is somewhat correct. I am also entitled to trust, without strict evidential requirements, the meteorologists' prediction that it will rain during a typical fall day in the pacific northwest. Because of their nonevidential nature, epistemic entitlements are reasonably suspect as justificatory sources in important epistemological debates (McGlynn, 2014). In this section of this chapter I will show how epistemic entitlements are even more problematic in the context of the warrants justifying the propositions and/or results stemming from a devise, namely computer simulations, deployed in scientific inquiry. Briefly, in what follows I will show that epistemic entitlements are inadequate to warrant trust in the computer simulations of computer simulations in science.

One way that philosophers of science have suggested explaining why we trust computer simulations appeals to the ways that epistemic entitlements work in other less controversial forms of inquiry (See for example Barberousse and Vorms 2014). For example, it might make 
sense to trust simulations in the same way that we trust our perceptual faculties and our memories, or in the same way that we trust expert testimony. We are entitled to base beliefs on the evidence of our senses or the testimony of experts in spite of not having full access to the underlying workings of the senses or the full understanding of the inner workings of those testifying. We do not need to fully understand the intricacies of the organs in charge of vision in order to take most of what is perceived by our eyes as true. Similarly, we do not have full access to the education and abilities of the expert. Yet, for all ordinary purposes, one is warranted in believing information stemming from either source. We have the right to believe-we are entitled to the belief - that the universe is over one billion years old without knowing more than a few paragraphs worth of cosmology. The argument from those advocating for entitlements in the context of computer simulations in science is that we are in a similar way entitled to the belief that the results of computer simulations are trustworthy. This, according to these views, is also in accordance with sociological and practical aspects of the way science and scientific communication happens. The assumption is that, sociologically speaking, for example, scientists do not have to fully replicate each other's extensive work in order to utilize each other's results in furthering their inquiry. They are entitled to trust each other. ${ }^{118}$ This is the case in an even

\footnotetext{
${ }^{118}$ While this may certainly be the case, sociologically and practically speaking, when multidisciplinary research is conducted, it isn't entirely clear that this is what happens or what should happen in intradisciplinary interactions. As a matter of fact, an easily available example of where this entitled-kind of trust does not happen is at specialized academic conferences. Rather than accepting each other's research via a non-evidential epistemic process, scholars gather to thoroughly inspect each other's work. A similar process is implied with reference to peer reviewed publications in science in which a significant portion of the publication is dedicated to a "justificatory" section. In such sections great efforts are undertaken to make the methodology and the processes involved in the acquisition of scientific results as transparent as possible. While some degree of trust is necessary, there is a big difference between the kind of trust necessary to trust that your peer is reading their paper correctly and accurately and whether or not their paper is correct and/or arrived at in a correct manner.
} 
more striking way, according to these views, when the results of scientists are communicated outside of academic contexts. Most of us, for example, have no choice but to trust what our science textbooks say. For Barberousse and Vorms (2014) denying this fact amounts to the dismantling of most knowledge-acquisition and production infrastructure that our species has relied on since written documents have been used to transmit information. Philosophers in this camp argue that something similar is the case for computer simulations: computer simulations are either like expert systems and we ought to trust their testimony like we would that of an expert, or they are like some of the elements that make up our perceptual capacities.

These views acknowledge that computer simulations are extremely complex in more than one way and that in general they operate in ways that are not surveyable by ordinary human minds. That is, computer simulations are often what is called epistemically opaque. However, this fact is taken as yet another reason why the notion of epistemic entitlements make sense in this context. Under these circumstances, some argue, it might still be reasonable to trust computer simulations given the assumption that everything is working smoothly (Beisbart 2017) just as it is ordinarily reasonable to trust our senses or the testimony of other people, even when we do not have full access to their inner processes. That is, unless you have evidence of facing a person whose reasonableness you cannot assume. When this line of reasoning appears in the philosophical literature, it frequently draws on insights from Tyler Burge's work. In particular it takes as a starting point Burge's view of how human beings ordinarily maintain a posture of acceptance in epistemic matters. Just as we ordinarily trust our senses, we also ordinarily tend to accept what other people say unless we have reason to disbelieve them. The justification, according to Burge, 
that enables us to acquire beliefs from others may be glossed, to a first approximation, by this principle:

"A person is entitled to accept as true something that is presented as true and that is intelligible to him, unless there are stronger reasons not to do so. Call this the Acceptance Principle. As children and often as adults, we lack reasons not to accept what we are told. We are entitled to acquire information according to the principle-without using it as justification-accepting the information instinctively.” (1993, p. 467)

Burge points out that this ordinary disposition to accept the testimony of others is a necessary condition for acquiring language, and for a range of other social phenomena. ${ }^{119}$ As far as science is concerned, failure to act in accordance with the Acceptance Principle would make rational collaborative projects of inquiry impossible. On this view, testimonial sources such as published research, standard pieces of scientific equipment, and expert opinion should be trusted by default as a precondition for the possibility of ongoing inquiry. Adopting unreasonably high epistemic standards makes inquiry impossible. It must be noted though, that —as noted above - there is a difference between unreasonably high epistemic standards and non-evidential warrants when it comes to what is acceptable in peer review processes such as academic conferences and publications. One could immediately point to the fact that at least internally, that is within the

${ }^{119}$ Burge's account of the Acceptance Principle (as he acknowledges) is very similar in spirit to the Principle of Charity, as it figures in Quine (1960) and Davidson (1973). The principal difference between these principles is the role that Burge's notion of preservation of content plays in his account. 
same discipline, acceptance of expert testimony is not the default position, nor is it a precondition for the possibility of ongoing inquiry. It is true that in rare occasions a reasonable amount of trust is needed in order to speed up the process of inquiry. It is also true that in some cases there is little option but to trust, i.e. some experiments and/or processes are too difficult to replicate by others. But these instances are neither the norm nor exemplars of normative behaviors in scientific inquiry. This is particularly the case if one believes that scientific inquiry should be at least a little more rigorous than everyday sense-data observations: we methodically document, test, repeat, ensure that others can corroborate by repeating the same procedures and then ask them to do so before we accept what is to be scientifically reliable. This is not something we do before crossing the street. This is part of what makes appeals to entitlements so puzzling in the context of scientific inquiry. There is a sense in which appealing for a nonevidential warrant to provide justificatory force to a reason to trust anything in science sounds highly dubious.

For Burge, acceptance is the epistemic default position for human beings in most epistemic practices and it grounds what he calls an a priori entitlement whereby a person is entitled to accept a proposition that is "presented as true and that is intelligible to him unless there are stronger reasons not to do so, because it is prima facie preserved (received) from a rational source, or resource for reason; reliance on rational sources—or resources for reason-is, other things equal, necessary to the function of reason" (1993, p. 469). Like Barberousse and Vorms a decade after him, Burge also talks of entitlement in terms of being a precondition for the possibility of knowledge and extrapolates what can be said about this in the context of sensorial 
experience into a context of more complex types of inquiry such as computer-assisted mathematical proofs.

Given that the Acceptance Principle serves as a necessary precondition for the function of collaborative projects of inquiry, it has been natural for philosophers influenced by Burge to imagine that a priori warrants can ground computer simulations in a similar way. This is particularly the case if one sees computational methods as mere extensions to existing mathematical methods, which is the case for many philosophers of computer simulations (Humphreys, 2004; Weinberg, 2012; Morrison, 2015). For such philosophers, the origin of the computer simulation as what can be characterized as a 'number cruncher' still play an important role even in contemporary settings. This is because while computer simulations become more and more complex, they are still tasked — at least in the case of simulations mimicking the behavior of complex dynamic systems — only, according to these views, with providing discrete mathematical solutions to computational models that are derived from mathematical models and the continuous equations therein (Morrison, 2015). As we saw in previous sections, the pipeline associated with characterizing the steps required to create a simulation from an empirical observation to a dynamic representation is often taken, by some (Morrison, 2015), to be constituted by purely formal methods. This in a sense explains why some would think that the warrants justifying the mathematical content that computer simulations manipulate would also be relevant when assessing the reliability of the computer simulations themselves.

Furthermore, even when there is an acknowledgement of the engineering and implementational challenges of computer simulations in these pipeline representations (as in Resch, 2017), these 
steps are assumed to simply preserve the formal content they transmit when the engineering is "done right." (Beisbart, 2017) As explained in sections above, why this is the case may be a simple case of folklore in which engineering is being idealized as applied physics and physics is being idealized as applied mathematics. Regardless, the advocates of this view of computer simulations sees them as nothing more than computing complex equations by following formal rules. As such, it is easy to see that, if this is all they do and they have been properly put together to achieve it, they will qualify as transparent conveyers of content whose warrants are of the a priori kind.

This is where Burge's work figures significantly in the discussion of computer simulations. Burge's reflections on computer-assisted mathematical proofs in the context of computer simulations emphasize the role of what he calls transparent conveyers of warrants. We have already discussed some details concerning the notion of transparent conveyers and content preservation. We will discuss the idea of transparent conveyers in more detail below, but for now it suffices to note that a transparent conveyer is one that does not modify the content it conveys in any epistemically relevant way. In the context of knowledge-generating content, this means that the processes/methods/devices by which it is conveyed do not change in any significant way the nature of the knowledge itself nor the warrants that serve for its justification. One immediate problem for the application of the idea of transparent transmission of warrants in simulation practices is that we cannot, in fact, be confident that computer simulations do not introduce epistemically relevant changes to the content being manipulated. For example, as Eric Winsberg notes, simulation involves what he calls motley sets of practices and technologies (Winsberg 2010). As we shall explore further below, the inhomogeneity of computer simulation as a 
practice means that the idea of transparent conveyance for warrants is not as applicable as it is in the case of computer assisted mathematical proof.

This is also the case given the inhomogeneity of components. But more importantly, as it turns out, the various and diverse components and processes involved in computer simulation do in fact change the content as it passes through them. Sometimes, it is by this very modification that they serve their purpose (as will be exemplified below in the case of discrete transformations of continuous equations to make them executable by computers). When we compare simulation to the process of computer-assisted proof, the latter clearly involves a more homogenous epistemic context (Barberousse and Vorms 2014). In computer aided mathematical proof one has a much better chance of ensuring the transparent transmission of warrants and the preservation of a priori content (Arkoudas and Bringsjord 2007; McEvoy 2008, 2013). This is in part because the very concept of proof pushes the endeavor of computer-assisted proofs to be more rigorous with the transparency and provability of the software and hardware components of the machines involved. Furthermore, given the specialized task and the nature of the task - a mathematical proof - special care can be justifiably taken to exhaustively test the hardware and software components of computer-assisted proofs. This is not the case-as has been extensively shown in other sections of this dissertation — with conventional development and use of computer simulations in science, particularly those that are developed in academic settings, which often have little adherence to industry standards. This is, of course, ignoring the fact that even in mathematical proofs, one may argue, and even in conventional mathematical proofs - as opposed to computer aided proofs - the process is not as transparent as one may initially assume so. It turns out that there are several reasons to doubt the conveyers of mathematical proofs even when 
such conveyers are peer reviewed human-made publications (Frans and Kosolosky, 2014). This is evidence that Burge's starting point may be more challenging than he leads us to believe. In particular, it may be strongly presupposing the reliability of many things that actually are subject to reasonable doubt.

This leads us to a more fundamental problem with taking Burge's strategy as the basis for the epistemology of computer simulation. The problem lies in his construal of our everyday reliance on the testimony of other people. As we shall see in the following sections, computer simulations and computational methods in general — as instruments deployed in scientific inquiry — are neither reliably transparent conveyers in all contexts, nor can they be regarded as equivalent to expert sources of testimony. Clearly there are occasions in which one is entitled to a belief independently of one's subjective grasp of the epistemic rights or warrants supporting that belief. We can be entitled to believe, for example, what the weather forecaster has told us independently of the state of our knowledge of meteorology (Dretske 2000; Williams 2000; Adler 2015). Given the epistemic entitlements described by Burge, we are frequently exempted from such justificatory practices as citing evidential support or of giving reasons more generally (Wright 2004; Burge 1993; Lackey 1999; Dretske 2000; Davies 2004; McGlynn 2014). However, there is one last note concerning epistemic entitlements that must be addressed before I can move on.

As mentioned above, the way epistemic entitlements are usually deployed in the context of computer simulations strongly relies on Burge's view that, barring a reason to doubt, one should take an acceptance attitude towards a reasonable source. In the literature regarding the epistemic status of entitlements, however, entitlements are not treated as monolithic and homogeneous type 
of warrants that simply give the receiver of information a right to hold a belief that what is transmitted can be trusted (Wright 2004; Lackey 1999; Davies 2004; McGlynn 2014). It is unclear whether the epistemic entitlements at play in the views described above constitute a reason to trust the results of computer simulations or reasons not to doubt the processes by which computers simulations arrive at their results. If the former was the case, then an argument could be made that given non-epistemic reasons-such as resource constraints, lack of a better option, etc. - we have the right to trust the results of computer simulations. This is however, not strictly speaking an epistemic warrant, but a warrant none the less (Dretske, 2000). If the latter was the case, however, one would wonder how it is that such a warrant was acquired. How is it that we have a non-evidential right not to doubt the reliability of a process/method/devise deployed in science? This is a sort of entitlement that even those who really want us to trust in the results of computer simulations would want to stay away from. For them, a warrant that speaks to the latter case, that of not doubting the processes by which a computer simulation arrives at its results, is warranted by, in their view, the vast empirical evidence on their behalf and not by some rational precondition.

In any case, in the sections following the next one I will discuss when we are legitimately exempted from justificatory practice in more detail. For now, however, I will focus on the details of why exactly computer simulations fail to meet the criteria as both transparent conveyers and as experts providing testimony. 


\subsection{Transparent Conveyers, Expert Testimony and Computer Simulations}

In the following sections I offer a series of arguments that directly undermine the deployment of epistemic entitlements, exemplified above, as an adequate justificatory source for our reliance on computer simulations. The first argument concerns the treatment of computational methods, processes and components underlying computer simulations as anything resembling transparent conveyors. For many reasons, detailed below, I will show that they are not. The second argument will also show that computer simulations and their associated components and processes should not be treated with a similar epistemic position as that with which we treat ordinary epistemic practices. In particular, I argue, computer simulations are not - like perceptual or testimonial sources may be - the kinds of epistemic sources whose well-functioning should be taken for granted; that is, unlike certain epistemic elements of inquiry, computer simulations do not enjoy having the status of a precondition for the acquisition of knowledge, nor do they represent a source of possible knowledge whose functioning should be taken as evoking no reason for doubt.

A second and conclusive argumentative section will follow, which will show via an example that, when it comes to scientific inquiry and computer simulations, a pragmatist position is ill equipped to capture the superior normative expectations of science vis-à-vis everyday epistemic practices. In this example, I show that what science is after is, at the very least ${ }^{120}$, slightly more

${ }^{120}$ Although my personal view on the requirements and the normative aspect of scientific inquiry is slightly stronger in that it calls for a full view of scientific enterprise as an enterprise of understanding and/or elucidating the way the world is and/or works, for my argumentative purposes here only a minimum threshold claim is required. That is, for the sake of the argument in this section the only thing required to show is that scientific inquiry is, at the very least, 
than what a group of oracle-believers requires of their predictive tool. If we are to make sense of our reliance on computer simulations for genuine scientific inquiry and for socially consequential decision-making strategies, such as policy and protocol design, simply expecting them to work and/or appealing to their predictive power are not epistemically satisfactory approaches.

\subsubsection{Computer Simulations are Not Transparent Conveyers}

Following Burge's application of the Acceptance Principle to the epistemology of computerassisted mathematical proofs, Barberousse and Vorms (2014) argued that the epistemic warrants supporting our reliance on computer simulations are not intrinsically constituted or enhanced by appeal to empirical evidence. They argue that the warrants behind our trust in computational methods can and do take the form of Burge-style entitlements. Just like other devices whose role, like that of memory or perception, is to transmit and preserve intact information, computational methods that manipulate mathematical content appropriately should be considered transparent conveyers (Burge 1993; Barberousse and Vorms 2014). In a transparent conveyer if the propositions being conveyed are justified a priori, for example, then this justification will not change from a priori to empirical. Following Burge, this is called content preservation. ${ }^{121} \mathrm{~A}$

meant to be a slightly better external methodology of record meant to overcome direct observational underperformance of everyday epistemic sources such as perception, memory, etc.

121 Though they acknowledge that there is a substantial difference between computer assisted mathematical proofs, such as the ones Burge focused on and complex computer simulations in terms of content preservation, they justify the aprioricity of a scientist's entitlement to trust a simulation in virtue of a second strategy (which we will inspect in detail in the following section): trusting computer simulations, they argue, is like trusting expert testimony: you do not need full and transparent access to the experts' thought process to trust it. Though expert testimony may be fallible, for this view, casting a general doubt on the practice absent specific reasonable doubt can be seen as 
method, process, or devise ensures the preservation of the content it transmits by not introducing any epistemic warrants that do not belong to the kind of warrants that the content is already justified by. If I was, for example, conveying to you the result of a geometrical theorem but in the process of doing so I appealed to the authoritative social position of Pythagoras as reason to endorse its results I would have in fact have introduced into the transmission of the theorem's propositions an epistemic warrant (appeal to authority) whose nature (a posteriori) is distinct from the warrants of the content being transmitted (which are presumably of the a priori kind given that we are talking about a geometrical theorem). If in contrast, I do not appeal to Pythagoras' authority and solely rely on my assumedly well-functioning memory I ensure the content is preserved in Burge's sense, and I and my well-functioning memory serve merely as transparent conveyors of geometrical propositions.

From this perspective, a warrant for belief can be a priori even if the manner in which one attained the warrant depends on some contingent fact about the world, such as the fact of having a brain or some particular perceptual capacity, even memory. The fact that a human being needs a brain to do arithmetic is irrelevant to the justification of an arithmetical proposition. One implication of this position, is that a cognitive capacity such as memory "is no more intrinsically an empirical faculty than it is a rational faculty. Its function in deductive reasoning is

irrational. This is in part because it is seen as undermining much of what we take to be reasonably acquired knowledge. It is also deemed irrational because it is assumed to be made in the absence of a reason to doubt. As we shall see below, that this absence is the case is not immediately obvious in scientific inquiry and particularly when dealing with the sanctioning of an instrument for scientific purposes. 
preservative" (Burge 1998). ${ }^{122}$ In other words, when memory does what it is supposed to do it conveys information without altering it in any epistemically relevant manner.

However, as Burge acknowledges, when it comes to the transmission of information outside one's own cognitive processes things are more complicated. Although Burge believes that there are similarities between the memory example and the way we can gather knowledge from other sources (from testimony or otherwise), he acknowledges that it is "only in special cases that a priori knowledge can be preserved through interlocution" (Burge 1998, p. 5).

It is very important to note, for example, that Burge's account of content preservation and transparent conveying requires that the recipient already has reason not to doubt the source. ${ }^{123}$ Much of Burge's argument relies on the intelligibility of other minds, and the inescapable need for trust in testimony as a condition for the possibility of the most basic epistemic and linguistic practices. However, on my view it is crucial to recognize that scientific inquiry is not a basic epistemic practice but rather a very special cultural practice that is designed, in part, to overcome the evident limitations of our ordinary epistemic conditions. ${ }^{124}$ This particular point will be

\footnotetext{
122 Similarly, when we rely on our senses, we grant that when they are working the way they are supposed to they transmit information without altering it. That is, as explained above, they are transparent conveyers. Thus, though one can acknowledge their fallibility, in the absence of a plausible reason to doubt their well-functioning, it is rational to rely on the senses (Burge 1993).

${ }^{123}$ As we will see below, and briefly mentioned above, whether one has reason not to doubt, no reason to doubt, or reason to trust represent significantly distinct challenges for this prerequisite.

124 Philosophy is another social practice that sets abnormally high epistemic standards. In the philosopher's case, we aim high with respect to what should count as a rationally persuasive argument.
} 
explained below, but for now suffice it to say that at the very least, the scientific enterprise aims to overcome evident and ordinary limitations concerning memory (by writing things down), observation (by repetition ), and possible bias (by efforts in transparency and reproducibility). Furthermore, when the issue is trust in a technical artifact whose prowess and adequacy is still very much on trial, we are already far removed from basic epistemic and linguistic practices. At the very least, it should be acknowledged, the preconditions for the possibility of knowledge were well in their place by the time computer simulations came about. That is, knowledge is evidently possible without them.

Content preservation, furthermore, fails in an immediate sense in the context of instruments deployed in the aid of inquiry. That is, using the notion of content preservation as a strategy to justify our reliance of a method/process/device fails in how the warrants that underwrite the content being manipulated by scientific instruments are inadequate to warrant trust in the instruments themselves. Even if the content being transmitted is justified via priori warrants, that the method of transmission is adequate and/or capable of transmitting such content, that it can do so transparently, and/or whether the warrants that justify our reliance on such method are of an a priori or an a posteriori kind are questions whose answer must come from independent epistemic evaluations from anything there is to say about the warrants that underly the content being transmitted. In other words, it is not clear that the warrants that justify the content in question are/can be the same as the warrants that justify a conveyor. We should further note, for example, that often the warrants that justify our reliance on transmitted content have only an asymmetrical relation with the warrants that justify our reliance of the method of transmission. This is particularly the case when the content is already justified or justified via a priori warrants. 
Consider, for example, that in some instances the justification of our reliance in some content is negatively affected if there are reasons to doubt the transparency of the conveyor. If you are a known delusional neighbor for example, I will have reason to doubt that what you just said about the Pythagoras theorem is in fact true. In an even simpler situation, if I do not consider you to be a full expert on Pythagorean matters, I may receive your information concerning the relationship between heights and lengths of triangles with a reasonable suspicion of alteration. So reasonable doubt on the method of transmission has an immediate negative effect on the warrants for trust of the content. By contrast, while reasonable doubt regarding the warrants of the content can also have some effect on the reasons for trust of the method of transmission, it is not to an equal degree. We can see this when scientists take a second look at their inputs way before they cast any doubt on their method of analysis. Whether or not a method of transmission is a reliable method is still an open question even when the justificatory force of the content being transmitted is a closed matter. Just because a scientist is dealing with algebra does not mean that they are any more justified in trusting the device they are using to manipulate it (an abacus, an analog machine, or a digital computer) or that the same warrants justifying the content transfer to or have much to say regarding the warrants related to the reliability or trustworthiness of the device. Similarly, and this is an important detail concerning the asymmetry mentioned above, if the device is warranted then the reasons for our reliance in the content being transmitted is in fact enhanced. If I know for a fact, and ahead of the fact, that you are a particularly brilliant mathematician and you tell me some truths about the relationships between numbers I have at least one more reason to warrant my belief in the propositions conveyed. In this sense, while the warrants justifying the content have little to nothing to say about the warrants justifying our reliance on the devise that transmits it, the warrants justifying our reliance on the method of 
transmission have a larger impact in the warrants that justify our accepting the content being transmitted. This important considering computer simulations since we can say that just because computer simulations solve for mathematical equations (even if we were to accept that that is all they do) this does not do much for the reasons to trust the results of the computer simulations themselves, while having reasons to trust computer simulations does in fact provide further warrants for the justification of our reliance on their results. As we will see below, computer simulations are not transparent conveyors, but if we are to trust the arithmetic results of computer simulations (that is once they are transmitted by a mediator), if we are able to warrant them, it is in fact because of the introduction of further warrants that do not belong to the kinds of warrants providing justificatory force to the content they manipulate. It will be because there are other evidential warrants providing justificatory force. That is if there are any at all.

Unlike perception and memory, or even the human tendency to trust others, the introduction of computational methods into scientific inquiry has been the product of artifacts and practices whose reliability has involved a gradual process including laborious efforts on the part of scientists and engineers (Winsberg 2010). As Evelyn Fox-Keller points out, contemporary uses of computational methods in science are the product of massive collaborative efforts since the Second World War involving trial and error approaches to practical problems (Keller 2003). As Winsberg argues, the development of computer simulations includes an array of distinguishable evidential benchmarks and other features such as considerations of fit, calibration, in addition to extra-theoretical and extra-mathematical engineering practices (Winsberg 2010). These practices involve both hardware and software innovations that are familiar parts of scientific practice but are seldom discussed in the epistemology of science. From architectural considerations for 
optimal processing to mathematical discretization, justificatory practices involving computer simulations involve facts about the history of their successful deployment but also the appropriate management and assessment of uncertainties, errors and calibration procedures (Ruphy 2015). These sanctioning processes, and therefore the computer simulation itself, cannot be regarded as transparent in the sense required by Burge. ${ }^{125}$ As mentioned above, the kind of homogeneity that transparent conveyers of epistemic warrants require is simply not available. For example, given the role of engineering constraints, implementation, discretization, questions of fit, calibration, and countless other non-explicit features of computer simulation it would be a mistake to think that the warrants underwriting our trust in these artifacts are simply derived from the formal character of the computer code that these systems run.

Consider the well-known features of discretization techniques. Many computer simulations are the result of a process that includes the transformation of differential equations in a mathematical model into expressions that represent approximate values for specific finite spatiotemporal states of a system. ${ }^{126}$ Discretization techniques involve determining practical ways of implementing

\footnotetext{
${ }^{125}$ As we will see later, they cannot be ignored either, as is the case when the justificatory force behind their sanctioning is said to be drawn from a non-evidential warrant. What ought to be done is to take these benchmarkings seriously and ask whether or not they are representative of a theoretically grounded process that included very carefully curated data in addition to the empirical trials mentioned. Only then, do these qualify as good reasons to support our trust in computer simulations. As it turns out and as I will argue later, this may not be the case. If so, computer simulations may not be granted entrance in to the canon of laboratory instrumentalia.

${ }^{126}$ One can think of discretization as trying to approximate a circle by drawing one regular polygon after another with more sides each time starting form a square. Of course, a square is a terrible circle, but a polygon with millions of sides may be visually indistinguishable for practical purposes. Nevertheless, at each point, one is not drawing a continuous curve but rather a series of straight lines at an angle from each other.
} 
calculations in manageable chunks. This transformation/translation procedure introduces epistemically relevant decisions on the part of the modeler that are distinct from the original mathematical model. In fact, sometimes, the discretization techniques are of such complicated nature that mathematicians specialized in conversion methods are enlisted in order to achieve some of the mathematical transformations required. In such cases, it isn't only the idiosyncrasies and/or available tools of the modeler, or those of the coder, that come into play, but also those of a whole other individual or team of specialists. That is, often the reason a specific set of equations is chosen in the mathematical model has nothing to do with the challenge of approximating the model via a computer simulation. Computational constraints such as whether a machine can handle the mathematical dynamics observed in the world need not be a constraint on mathematical models. By contrast, the choice of specific discretization techniques in a computer simulation will be responsive to the practical necessity of implementing a model in a digital device. In fact, when it comes to discretization techniques, the decision to select one technique over another is often a matter of engineering trade-offs (Winsberg 2010, pp. 12, 23).

Discretization techniques, a fundamental aspect of computer simulations, undermine the possibility that computer models can serve as transparent conveyers insofar as they alter the nature of the epistemic justification of the content being manipulated. And this is so even when one grants that they are working as intended, as Beisbart (2017) suggests. Further, even if the reasons to trust the results of the equations in the original mathematical model are grounded in well-established theory, or are supported a priori on purely mathematical grounds, the introduction of discretization techniques involves an engineering element that alters the justificatory considerations involved in a computer simulation of the mathematical model. 
A further aspect to consider is that the actual numbers related to the discretized mathematical models run by the components in computer simulations are constrained by a limited memory array with restricted available digits to express a result. That is, several important computer components are often tasked with storing computational results within memory arrays that have a limited number of slots. Because of this, the actual results of a numerical computation are rounded up and/or down to the most relevant number of digits that can fit into the array. This happens on every component, on every machine and on every computation of every simulation. At every turn, from one relevant component to another, the results are rounded up or down in order to fit the constraints of the machine, the architecture or the implementation model of a computer simulation. This introduces a significant discrepancy, or error, between the actual results of a computation and the results that can be conveyed given the memory constraints of a component. While methods exist to assimilate the numeric discrepancies generated by such processes, the fact remains that the rounding up, the error correcting and/or the approximation methods represent an extra manipulation of the content in epistemically meaningful ways. ${ }^{127}$ 
Some such processes are in fact epistemically opaque and do not offer easily identifiable ways to correct for their artifactual results (Kaminski, 2017).

Thus, in a very basic sense, computer simulations cannot count as transparent conveyers given Burge's characterization because justificatory elements distinct from the ones warranting the original content do in fact enhance, decrease or constitute the epistemic warrants of the manipulated content (McEvoy, 2008). ${ }^{128}$ Furthermore, as we noted above, computer simulation involves independent epistemic warrants at different stages. Consider the following. Even if the mathematical model is fully warranted and works as intended and even if the discretized version of the model also works as intended there is no reason to think that we have reason to trust the latter because of the former, as explained above.

When a discretized model is ultimately implemented in a device, for example, it requires yet another epistemically relevant transformation in the process of coding. In coding, considerations of fit, trust and/or reliability of a given algorithm will depend on independent factors from those

do not belong to purely formal, mathematical, strategies. How we judge these strategies - as epistemically sound or not, trustworthy or not - is independent from the way we judge the mathematical content being manipulated by the machines in questions. As such, they introduce a relevant epistemic modification to the content they are conveying. If this is multiplied by the several components and/or machines involved in computer simulations it is easy to see how the rounding of results represents an epistemic challenge to the notion of transparent conveyors in computational processes.

${ }^{128}$ McEvoy in his response to Tymoczko and Kitcher on the aprioricity of computer assisted mathematical proofs concedes as much by saying "What determines whether a proof is a priori is the type of inferential processes used to establish the conclusion of that proof. If the method of inference for any of the steps in the proof is a posteriori, it is a posteriori" $(2008$, p. 380$)$. 
involved in the discretization process. Unlike discretization techniques that involve established techniques and theories, code is often the result of highly idiosyncratic problem-solving approaches. Coding practices are error-prone. Consider for example the way many significant software bugs are 'patched'. That is, they are not 'fixed' per se, as in actively engaging with their malfunctioning components or erasing an erroneous block of code. Rather, code is added that 'patches' the original code by superseding previous functionality. These patches almost always introduce their own new bugs, making the process of assessing the reliability of the software even harder than it already was. In the process of patching, as is often observed, software, which is at the core of any and all processes of computer simulations, tends to grow over time merely because it can (Holzmann 2015). ${ }^{129}$ The apparent inevitability of errors in the practice of coding is an empirical reason to decrease trust in any content manipulated via computational methods. ${ }^{130}$ This is particularly the case if the warrant in question is an epistemic entitlement. Thus, even if one grants that a computer simulation seems to work as intended, it cannot be regarded as a transparent conveyer in Burge's sense.

If, as Burge suggests (1998, p. 4), one of the main issues to address is whether an entitlement or justification has any independent justification apart from empirical evidence, or as Barberousse and Vorms (2014) put it, whether the justification of a warrant is in any way constituted or

\footnotetext{
129 This is especially the case now that memory has become so inexpensive in modern computing. The 'true' command in Unix, for example, which originally consisted of an empty file with nothing to execute grew to nearly 23,000 bytes from 1979 to 2012 .

${ }^{130}$ See Horner and Symons (2019) for a review of the empirical literature on software error. They show that there has been a relatively consistent level of error reported in empirical studies from 1978 to 2018-for every 100 lines of code between 1 and 2 lines of code contain errors. (See also Symons and Horner 2017).
} 
enhanced by empirical means, then the answer concerning the warrants underlying out trust in computer simulations is clear. Typically, the results of computer simulations have been through a process that definitely alters whatever formal content they have manipulated by a series of processes that are dependent upon empirical considerations. In very practical terms, for instance, software design is often limited by empirical and engineering constraints. Furthermore, some of these constraints are such that alteration is the way they work, as in the case of discretization methods and coding that underly the transformation from mathematical models to computer implementations of a target simulation.

In an effort to provide a more refined version of the Burge-style entitlements account, Beisbart (2017) acknowledges some of the practical complications that we have noted, but argues instead that warrant transmission takes place from one stage in the simulation process to the next. On his view, the results of computational methods can be said to provide knowledge in virtue of a sequence of inferential transfers from one level of propositional content to another. That is, at each step in the simulation process, there is a result, a proposition to be considered: from target phenomenon to mathematical model, from mathematical model to computerized model, from computerized model to computational implementation, and from single implementation to iterations (Beisbart 2017, pp. 160-162). The important point here is that the warrants of the previous step are not merely transferred to the next step as providing justificatory force within the next step. There is also not too much hinging on whether or not the warrants are unaltered. Rather, once each step is concluded with its own warrants (whatever they may be) we are warranted in inferring that we can move on to the next step without having to exhaustively investigate the warrants of the previous step. In this sense, there is still an assumption taking 
place, and there is still an entitlement kind of warrant at play as we move along the process, but it is not playing the same role as it is with the views explored above, namely those of Barberousse and Vorms and other Burge-style arguments. At any given step our trust is justified if we assume that that our methods are the adequate ones and we assume they work as intended. In other words, we are warranted in inferring from one stage to the next if we grant that each level is somewhat/somehow warranted to begin with. For Beisbart, an agent is "inferentially justified in believing a propositional result constructed from a computer simulation if she is justified in believing the dynamic equations used to feature the system under scrutiny and if she is justified to think that the simulation works as intended" (2017, p. 171). However, for the agent to be justified in believing that the simulation works as intended, all that is required is that the "epistemic agent has sufficient reason to believe that it does so" (p. 169). This "sufficient reason" in turn implies, the assumption that at every inference step, from mathematical model to final display, certain types of errors (round-off errors, modeling errors, hardware failures) are "excluded or are sufficiently small" (pp. 161-163). At every step however, trust is warranted with the proviso that there are no significant epistemic challenges (2017, p. 162). As we will see, on my view, this is exactly the open question that has not been and ought to be settled. In all these views there is the assumption that could assume or that we must assume the wellfunctioning of computer simulations in order to allow for scientific inquiry to be conducted through them. However, I have shown how the latter is not the case, we do not have to assume anything regarding computer simulations epistemic status (computer simulations are not like other preconditions for the possibility of knowledge, as some argue). Furthermore, the aim of this section is to show that the former, that we can assume that computer simulations work well, is also a questionable strategy, in particular because there is nothing that warrants this 
assumption. In other words, we have no epistemic reason to believe that the results of computer simulations are true. The project of sanctioning computer simulations, whether in practice or in normative projects such as the present one is precisely to establish a criterion by which they can be assessed to be well functioning, and then apply that criteria and figure out whether their place in scientific inquiry is indeed warranted or not. On Beisbart's account, a scientist is ultimately justified in believing a proposition derived from a computer simulation because what the computer itself has done is drawn inferences from the propositions of a discretized conceptual model (2017, p. 165). The scientist draws the final inference that ties the results back to the target phenomenon by assuming that at any given step the methods involved worked as intended. Note that Beisbart recognizes the distinction between warrants supporting the content of simulations and warrants supporting our reliance on their results. Strikingly, his analysis highlights precisely the relevant empirical and historical considerations that must ground our trust in computer simulations, namely a strong foundation in theoretical principles and processes that ensure that well curated data are put in to them.

As we have seen, the practices involved in simulation processes are such that they are typically not the product of transparent conveyers even when they seem to work as intended. The reasons why each of the steps can be said to be reliable and/or trustworthy are independent from the step before or after it, offering no single kind of warrant transmission that spans the entire process of computer simulation. Warrant transmission in purely epistemological contexts, without the addition of complex technical artifacts as mediators, is already a complicated philosophical issue (Beebee 2001; Davies 2004; Pryor 2012; Moretti and Piazza 2013). Furthermore, the question of whether epistemic entitlements can at any point provide the justificatory force required from 
genuine epistemological processes poses important challenges that are not easily established. Whether or not, for example, an epistemic right such as a non-evidential entitlement can ever become a sufficiently-enough justificatory source to generate knowledge is often casted as epistemic alchemy and regarded as highly suspicious in epistemology (McGlynn, 2014). Can knowledge proper, usually thought to be the product of evidential efforts, suddenly emerge from a series of steps that prominently figure non-evidential warrants? If this epistemic alchemy is the case then we all know a lot of things without having done proper evidential diligence, which may be the case. If epistemic alchemy is not possible then it means that only some of us know some things properly and the rest of us trust but not know. This is also strongly plausible. While this may be a conservative view on what knowledge really is out there and who really does know something, it strongly resonates with the intuition that some of us really do know physics while others do not. This is a restrictive view I am willing to take. On the other hand, if epistemic alchemy is accepted in order to rationalize the acceptance of computer simulations as knowledge-generating instruments, then there is in fact no distinction between those that go through strenuous evidential efforts to ensure that something may be the case and those that do not. This applies to our discussion of computer simulations and their results. And this seems to me highly unlikely.

As I will explain below, whatever reason we have for trusting the results of one stage in a simulation as we move to the next should generally not be ad hoc; it should not be based solely on the factors that are unique to particular instances of a simulation practice itself but should be the product of established theoretical principles and engineering standards. The methods and technologies used in practice are not simply deployed in an unchanged form across different 
applications, processes and platforms. They are modified, often in non-trivial ways, to suit the task at hand. Throughout these modifications there are ways in which one can establish benchmarks that help to sanction the results of a simulation. These might involve, for example, repeated runs and internal comparison, comparison to outputs of other simulations, or most importantly, comparison to real-world data (Symons 2008; Winsberg 2010; Gramelsberger 2011). Thus, when computer simulations can be trusted it is because of their adherence to theoretical principles, empirical evidence, or engineering best practices and not because of their output alone. Like other instruments deployed in scientific inquiry, computer simulations do not simply inherit their warrants in virtue of their manipulating formal syntax in a rule-governed manner. They also do not directly inherit them from the fact that theoretical principles themselves, or the data analysis methods themselves, are warranted. Rather, just like instruments, they must invoke their own set of warrants (which in turn must include considerations of coherence with independently justified theoretical principles and empirical observations). It must be noted however that unlike other instruments in science whose implementation and reliability has often been determined through processes that have depended on well-established traditions and principles, digital computers and computer simulations have had a relatively short period of use. And while some scientific instruments have been adopted quickly and successfully in restricted domains of inquiry (e.g. the electron microscope, MRI, etc.) computer simulations are applied so ubiquitously that the assessment of their success is not as straightforward as it is for many more targeted scientific instruments.

As mentioned several times above, in addition to the importance of recognizing different kinds of epistemic practices in different stages of the construction of the simulation, we also need to 
consider that even when the foundational components of the simulation are sound, the processes of combining them into a working application can fail in many challenging ways. To put this point simply, we can say that the inference from warrants at the level of "parts" to warrants at the level of the "the whole" computer simulation is not sound. The reason that it is a mistake to regard an epistemic warrant as being successfully conveyed from relatively simple, "componentlevel" inferences to the behavior of the simulation as a whole, even in cases where the components of a software system are well understood, is because of the role of software engineering itself. Software, which is an essential part of any general-purpose computing system, is a source of error insofar as it is created via human engineering and is built to serve human purposes. Human engineered software contains errors at the level of coding and vagueness at the level of specification (Tymoczko 1979, p. 74; Fresco and Primiero 2013). These errors mean that normal notions of epistemic-warrant-transmission from elegant and well-understood foundations to the behavior of aggregates of these components simply do not hold (Winsberg 2010). In other words, as Stéphanie Ruphy notes, "the computer simulation does not simply inherit" its epistemic credentials (2015, p. 139) Rather, its reliability is rather the product of a range of diverse sanctioning processes.

As we have seen in this section, our trust in computer simulation takes place within the motley traditions of engineering and scientific practice. The actual process of building a simulation involves distinct stages, each of which has its own epistemic standards and warrants. This heterogeneous context makes the idea of transparent conveyers inapplicable to the epistemology of computer simulation. As I will show in the final section of this chapter, our trust in computer simulation is fundamentally different from the epistemic relation that a group of oracle believers 
have to their mechanical oracles. Furthermore, as we will see in the next section, in themselves, computer simulations are not what should be taken as good sources of testimony. This is because it is their credentials that we are trying to establish. To treat them as possible sources of expert testimony is already granting them the 'expert' part of expert testimony that makes any testimony have the desired credentials for anyone to be entitled not to doubt it, if one was ever to appeal to such a position or epistemic warrant.

I have argued that there is a non-trivial epistemic difference between formal systems and computer simulations. The epistemic status of computer simulations is more akin to that of scientific instruments, than to mathematical proof.

\subsubsection{Computer Simulations are not themselves sources of expert testimony}

This section distinguishes between the ordinary epistemic thresholds involved in trusting others and those that ought to be deployed in trusting computer simulations as expert sources in scientific inquiry. According to Burge-style views of epistemic entitlement, when we are dealing with a given piece of testimony that we have no plausible reason to doubt, the default rational position is to accept it as truthful (Burge 1993). If, for example, I hear that weather models predict that a hurricane is likely to hit my city, it would be rational for me to heed the meteorologist's warning without rigorously investigating the methods and evidence supporting the prediction. In this scenario, it would seem that I am trusting the model in the same way that I would trust an expert. This, however, is not the right way to understand the non-scientist's attitude towards the meteorologist's prediction. First, we would argue that in the weather 
forecasting case I do not trust the models as experts. Instead, I trust the judgment of the human meteorologists with respect to the models in question. But even then, the reasons why I trust the human meteorologist include a wide array of epistemic warrants of different kind and justificatory force. I would for example, at the very least, trust one meteorologist over another depending on the journalistic trustworthiness of the station broadcasting the predictions. If so inclined I could appeal to past predictions and their accuracy or also reference to the sort of education one meteorologist has received over another if I had the option. Hence, the 'expertise' of the human meteorologist would have been established by me or others I trust (news sources, institutions, etc.,) before I trust the meteorologist herself. Whether computer simulation have been similarly vetted is what we are trying to find out, not what should be assumed when we are wondering whether to trust their results or not.

Second, while it is reasonable for me to regard the meteorologist as a reliable source of testimony (Borge 2003; Jenkins 2007), it would not be reasonable for the meteorologist him—or herself to maintain this epistemic attitude towards the computer simulation. It is reasonable for us, in ordinary life, to trust the weather forecaster insofar as they have the endorsement of experts. Neither we, nor the weather forecaster should treat the simulation itself as an expert. Again, the simulation in itself is not self-validating. When things go well, the community of experts and the tradition of using simulations in successful scientific practice grounds the confidence of experts in the use of simulations.

A scientist may certainly be justified in relying on the computer simulation's pronouncements once it is integrated into her practice or into the practice of other experts (Audi 1997). As 
explained above, this is a long and gradual process. Sometimes it is a process that can take decades. It is never the case that a scientist should give as a reason to trust a simulation the claim that the simulation is an expert. To treat our trust in simulations by analogy with our trust in human experts, would be to miss the actual warrants that ground the trust granted by human experts to simulations. When non-experts take the testimony of experts seriously, they are not granting that same level of credence to the tools used by the experts they trust. For example, they might not even know of the existence of those tools. In our case, most consumers of the weather forecast simply trust the experts to interpret computer simulations and other tools for them. In other words, the one thing we humans assume when we trust other humans in the way described above is that the human in question has done due diligence and passed all the credentialawarding procedures that allow them to interpret and then convey, somewhat transparently, what the science says about the weather with an instrument as an enabler. We do not assume the instrument to be the conveyer by itself or the one that merits the trust by itself, and certainly not by default.

Justification to trust testimonial evidence comes from observing a "general conformity between facts and reports [by which] with the aid of memory and reason, we inductively infer that certain speakers are reliable sources of knowledge" (Lackey 1999, p. 474). It is the conformity between facts and reports that does the heavy epistemic lifting when we trust experts. The facts and reports are analogous to the theoretical foundations and empirical processes in the context of more advanced epistemic practices such as science. What is described above may be the case for those humans that we consider experts, but it certainly is not the case for computer simulations. For simulations, the reasons that experts would count them as reliable sources of knowledge is 
their relationship to theoretical principles and engineering best practices in addition to their predictive successes. For example, a reason to trust a given computer simulation of a system is not merely because it is able to predict a given state of the system, but rather because it does so in the appropriate way - in conformity with the laws of physics, in conformity with engineering practices that are amenable to error assessment, etc. (Resnik 1997; Symons and Alvarado 2016)

All of this is to say that the task for experts in the evaluation of computer simulations is fundamentally different from the consumption of computer simulations by non-experts. Even if we had to accept that some of us, most of us, have to somewhat trust computer simulations in as their results are transmitted by scientist and others, this is not the case for those involved in the development and deployment of computer simulations. Furthermore, it is definitely not the case for those of us seeking a philosophically sound argumentative strategy to establish their epistemic status. Non-experts depend on experts to have certified the relevant computer simulations as worthy of attention. The testimony that non-experts are trusting is the testimony of expert communities, not directly the output of their simulations. A scientist relying on computer simulations should not trust their results simply as a matter of a default rational position. A philosopher trying to establish the normative guides by which a novel scientific instrument is to be deemed epistemically viable in the context of scientific inquiry has even less reason to appeal to such a strategy. 


\subsection{Scientific Inquiry, Everyday Epistemic Practices and Computer Simulations}

I have argued that Burge-style approaches to epistemic entitlement are inappropriate in the context of computer simulations when it comes to scientific or other high-stakes applications. However, I have also contended, albeit briefly, that science should be, and often also is organized around more stringent epistemic norms for the evaluation of computer simulations. In fact, it might even be the case that since time constraints are less pressing in much of science than they would be in, for example, military or policy contexts, scientists have the luxury of being even more rigorous and demanding with respect to the epistemic standards governing their devices. In saying this I am not discounting the serious time constraints faced by individual scientists in their careers e.g. the need to publish novel findings, the pressure of funding agencies, or the pursuit of tenure requirements. Rather, I am referring to scientific inquiry as a series of methods aimed at furnishing the best available understanding of our world. As such, scientist (from astrophysics to geology to biology- and some social sciences) can and ought to ensure a rigor in their methods that the nature of industry and war seldom afford.

In this section I want to expand on some fundamental differences between basic epistemic practices - such as the ones described by Burge and others appealing to perceptual and testimonial capacities as model epistemic practices in scientific inquiry—and epistemic practices that that have emerged from more sophisticated normative standpoints, which in turn require them to transcend common limitations of basic practices. In particular I want to point to the fact that scientific inquiry is not like perception and/or other basic epistemic practice. There are two main points that make this case. The first one is that while there may be reasons for some to trust 
either perceptual and/or testimonial sources in the way that entitlements suggest, these entitlements may not be of an epistemic kind. Clearly, there are instances in which time does not allow for someone to fully survey an expert's propositions and must trust the contents being conveyed; other times one may not have full access to the instruments or processes by which a team of researchers arrive to a conclusion (here I am thinking of the kind of equipment and procedures at the LHC) and therefore I have a reason to default towards accepting the findings that come from them. But note that these reasons, insofar as they can be deemed as reasons to trust, are practical reasons and not epistemic reasons. That is, they do not provide further epistemic justificatory force, just practical, or pragmatic, justification for me to fall back into a position in which I have no other option but to trust, say for the sake of moving on with a conversation, a project, or simply with citing my sources. This may be what is happening when philosophers appeal to entitlements in the context of scientific inquiry. If this is so, then maybe we can agree that sometimes there are reasons, other than epistemic reasons, that play a role in the development of scientific results and that this is what happens in the context of computer simulations. In my view, whether this can constitute the proper basis for a normative argument in the philosophy of computer simulations is highly dubious. The second argument that draws a distinction between epistemic norms at play in scientific inquiry and those at play in everyday epistemic practices consists in reversing the context of such norms and see if they are equally adequate for the opposite context. My claim is that epistemic norms at play in everyday epistemic practices are inadequate in scientific contexts. As I will argue below, one way to make this point is by showing that scientific norms are also completely inadequate in everyday epistemic practices. The conclusion is that there is a stark distinction between both. A very plausible explanation of this distinction is that it has something to do with a difference in rigor, 
adherence to theoretical principles, and a normative call for higher epistemic hygiene of one context over the other one.

Clearly there are aspects of scientific practice that are just like the epistemic practices that one would find in ordinary life. Scientists are people too, and in this sense, Burge's Acceptance Principle will have an important role in the way scientist speak to each other, convey casual information about their research to one another and ultimately rely on one another. We are not denying the fact that in order for scientists to engage in research, they need to trust one another, they cannot maintain an attitude of radical skepticism, and they need to make reasonable tradeoffs between time spent in various epistemically relevant tasks.

However, there are a few distinctions that ought to be drawn in this discussion. First, philosophical treatment of computer simulations should distinguish between the kinds of entitlement being appealed to in distinct contexts. Is the entitlement in question, for example, an entitlement to trust, or an entitlement to not doubt? ${ }^{131}$ Second, there are epistemic and nonepistemic entitlements that can be invoked as justifications to trust something. For instance, an epistemic entitlement might be playing a role in our decision to trust a medical device given our

${ }^{131}$ It is unclear, as noted above, the extent to which Barberousse and Vorms assume a negative (entitlement to not doubt) or positive (entitlement to trust) role for entitlement in their arguments. However, this particular paragraph focuses on the completely distinct categories between kinds of entitlements (a kind of warrant that gives someone $a$ reason to believe $(\mathrm{x})$ ) beyond their epistemic variety. 
belief that it may be beneficial to a patient. A non-epistemic reason to believe might involve our commitments to our affiliation group. There might be good social reasons to believe what a particular person says in cases where the matter under consideration is trivial or involves no harms or significant expense. If the cost of error is low, then arguably it is reasonable to simply accept the other person's position, in cases where the social (or other) benefits are important. A person may accept or reject some scientific hypothesis for social reasons. Perhaps this can be regarded as reasonable insofar as that person's individual commitments have little practical import. Her views for example on climate change, evolution, the biology of sex and gender, or other socially controversial matters have little real significance for either the practice of science or for policy decisions based on the scientific hypothesis in question. Insofar as their position on these matters causes no significant harm, it is at least understandable why consumers of science would choose to practice bad epistemic hygiene with respect to the science in favor of strengthening their position within an affiliation group. They strengthen their place within their social network by assenting to the consensus position of that group, perhaps even assenting to an exaggerated version of that consensus position. In those cases, as stated above, one may indeed have reasons to believe/trust, but they are not strictly speaking epistemic reasons (See for example Dretske 2000, p. 594). Epistemic reasons provide justification for the belief that a proposition is true. Having to accept somebody's proposition because of lack of resources to access evidential sources, or because they have a gun to our head does not meet this condition.

According to Dretske, for example, if what we are appealing to in accepting some proposition as true is to be regarded as a genuinely epistemic reason then the only grounds are its truth or probable truth (2000, pp. 593-594). The truth or probable truth of the values produced by an 
instrument deployed in the aid of science can only be a product of a reliability assessment, which is in turn distinct from personal justification (Williams 2000). That is, whether or not an instrument is reliable is not the same as whether or not we believe it to be so. Epistemic entitlement for computer simulations, should not be reduced to, or confused with the effort to provide what Dretske calls "a pragmatic vindication of the practices we use to fix beliefs" in place of efforts to "validat[e] the beliefs themselves" (2000, p. 598). Merely showing that a "practical purpose or need is served by accepting certain practice does not address the problem of our epistemic right to accept the individual beliefs that occur in this practice" (ibid). This is an important point that will be useful later as we discuss pragmatic approaches to the sanctioning of computer simulations. As we can see, an entitlement to trust does not necessarily mean a warrant to provides justificatory force to believe that something is true, or more likely to be so.

This is not a distinction made by Barberousse and Vorms and/or those deploying Burge style arguments of entitlement. If they are referring to entitlement as providing a reason to trust and they accept that this reason is not an epistemic one, then we can concede that there are indeed, often, reasons why scientist and non-scientist have no option but to trust one another's propositions. This applies to computer simulations. However, as we saw above, we should not confuse these reasons with epistemic reasons. In particular, that this (sociological, practical, etc.,) fact constitutes a normative epistemic claim concerning the way to go when dealing with the epistemic status of computer simulations is not immediately clear. Just because I have reasons to trust the results of computer simulations, does not mean that I have reason to believe the results of computer simulations are true, or accurate, or significant in the context of scientific inquiry. While this is far from a devastating blow to the use of computer simulations in science, what it 
does show is that there is a significant distinction in the kinds of epistemic entitlements and that this distinction has to be taken into consideration. This is particularly the case if we believe science to be fundamentally an epistemic endeavor and not merely a practical one. Epistemic endeavors require epistemic justifications.

\subsubsection{Higher Epistemic Standards in Science}

Scientists are held to a higher epistemic standard than ordinary epistemic agents for a reason (Nola and Sankey 2014). This is one of the reasons why they can serve the role of providing expert testimony. Consider Burge's own use of the example of Newtonian calculus (1998, p. 8). Burge's claim is that non-demonstrative reasoning can underwrite sound mathematical beliefs. An example of this, he argues, is that Newton's "knowledge" of elementary truths of calculus was available to him before formal explanations were available. However, consider too that without such formal explanations few would have considered Newton's knowledge to have any epistemic value. Similarly, Burge provides another example in which people accept the Pythagorean theorem solely on the basis of a diagram or the word of another without being able themselves to produce its proof. This acceptance only takes place in circumstances where no consequential inquiry is being conducted on the theorem itself, or in which the theorem does not play a central role. In fact, to accept the Pythagorean theorem in this way is to treat it on a par with, for example, one's unreliable neighbor explaining that recycling is collected with the trash every second week. The significance of knowing when to put out the recycling with the trash is (under normal circumstances) low enough, that believing one's unreliable neighbor is a reliable strategy. If it were very important, for some reason to know the recycling and trash collection 
schedule with greater certainty —imagine, for example, that we were the person in charge of the agency collecting the trash — then one should make additional efforts to determine the truth.

Burge's example of how one might come to accept the Pythagorean Theorem without really understanding the proof is convincing only insofar as we have relatively low standards for what we take to be true in mathematics. In ordinary life, it is true that our standards will often be low. There are cases where knowledge of the Pythagorean Theorem will have the same priority and role in our everyday lives as knowledge of the trash/recycling schedule. However, in the practice of science, our standards are higher and in the judgment of scientific evidence, a critical educated public should also hold important matters to a higher epistemic standard. Consequently, expert scientific testimony is not on a par with ordinary testimony and ordinary epistemic norms are not adequate to formulate an epistemology concerning scientific practice or the public understanding of science.

While no individual scientist is an epistemic saint, the scientific enterprise collectively seeks to ensure truth-aptness and reliability independently of the shortcomings of each individual practitioner. In relation to modeling and simulation, this has practical import. Humphreys, for example thinks that practitioners should always possess working knowledge of a given instrument and he argues that the background theory of its principles should always be at hand to the practitioner. This is not only because some instruments, for example, an MRI scanner, require this knowledge in order to be used effectively (Humphreys 2004, p. 38), but also because, the theory of how the instrument operates is "routinely needed to protect the user against skeptical objections resulting from ways in which the instrument can produce misleading 
outputs" (p. 38). Computer simulations are often used in contexts where highly demanding scientific standards are in place and where even ordinary well-grounded scientific practice falls below the threshold for acceptability (Ruphy 2011; Morrison 2015).

As a last point regarding the distinction between epistemic norms at play in scientific inquiry and those in everyday epistemic practice consider the fact that scientific standards would be regarded as unreasonably strict if we were to adopt the perspective of everyday epistemic practices such as normal decision-making, trust in perception, and credence with respect to the testimony of others. Not much would get done if we were to deploy epistemic norms taken from scientific inquiry in every day epistemic practices. This is because the requirement to accept something as part of scientific inquiry are substantially more demanding than those of our everyday practices. Consider for a moment a scenario in which I am wondering whether or not that white thing my son just threw at me is indeed a baseball on a direct trajectory towards my face. Consider another scenario in which I am wondering whether or not that car will stop at a red light so that I can go during my green light. Yet another scenario: imagine pondering whether or not that mango on your table is no longer edible. Now, consider applying the kinds of norms required for the acceptance of a scientific result in each scenario before you accept the results of a deliberation. A quick run through what that would look like should evidence the futility of the exercise. This is because scientific epistemic norms are absurdly demanding for everyday epistemic practices and therefore inadequate for such settings.

All these scenarios of course allow for distinct degrees of epistemic care. I have absolutely no access to epistemic, maybe even cognitive, resources in the first scenario before I make a move. I 
may be able to look a couple of times and assess speed variation in the second one before the pressures of my fellow commuters accumulate and make me step on the pedal. And I may be able to perform a couple of manipulations on the mango and deploy a few senses in the service of my decision. Yet, neither of these epistemic practices compares to or suffices for what scientific purposes demand. Looking twice is not the same as peer reviewed and/or precisionfabricated material deployed in laboratory settings. Deploying a few senses is not equivalent to performing distinct and exhaustive tests on a material or a phenomenon. This is evidence that satisfactory epistemic norms in every day practices are not on a par with even the most basic of norms in scientific inquiry. Consider the following. Let us imagine that an exaggerated level of care one would take in each scenario, right to the point before it all becomes absurd (i.e., we would not want to go to the extremes of requiring blindly controlled representative samples of traffic before we decide to cross a street). Then, imagine we switch whichever norms we have deemed epistemically satisfactory in any of the scenarios and apply them in a scientific setting. The question is whether they would be satisfactory at all. Most reasonable, even if exaggerated, levels of epistemic precaution taken in everyday life would not even come close to the procedures by which we ensure, or attempt to ensure, the norms practice in science. This should make it clear that the norms governing ordinary epistemic practices are not appropriate for the evaluation of scientific results. More importantly, it should make it clear that they are inappropriate to evaluate the results of a novel and opaque technical artifact such as computer simulations. 


\subsection{Pragmatism as Inadequate Epistemic Framework for Computer Simulations}

Imagine an oracle that produces reliable predictions but does not grant its users understanding or explanation concerning the system whose states it predicts or concerning its own inner workings. In this section I will argue that we should not, and (for the most part) do not treat simulations as oracles. As we shall see, warranted trust in the results of simulations is grounded in much more complex practices than simply deferring to a record of predictive success. In so far as a 'pragmatic' approach relies on making the case that predictive successes (however small or large they may be $)^{132}$ are the grounding warrants for trust in computer simulations, as Hubig and Kaminski (2017) suggest, these views will prove to be inadequate. The differences we note here between the mechanical oracle and the actual practice of simulation in contemporary science and engineering will help us to explain the limits of pragmatist and Burge-style approaches to the epistemology of computer simulation. I will explain, by contrast with the oracle, where we should look in order to understand how trust in actual simulations is and ought to be warranted. One implication of my account will be that some prominent uses of "simulation" in the social sciences - for example much of what is called agent-based modeling — may provide interesting sources of insight but will not count as trustworthy guidance with respect to fully sanctioned

\footnotetext{
132 Budig and Kaminski (2017) for example, seek to differentiate their pragmatic approach in computer simulations by emphasizing that their strategy needs only to focus on small practical engineering victories and not, as others suggest, in long term projects seeking convergence to a Truth. In their view, engineering successes constitute little truths in so far as they achieve the tasks at hand and these successes can be taken as the foundation for a "pragmatic approach to the epistemology of computer simulations" (ibid).
} 
scientific inquiry and therefore should not count towards efforts related to policy or other kinds of decision-making.

Nicole Saam (2017), for example, thinks that given that the problem of epistemic opacity can be a serious obstacle to the reliability of simulation outcomes, only those computer simulations that are generated and treated as thought experiments (as opposed to laboratory experiments) should be considered in the social sciences. Simulations treated as scientific experiments have at their core, significant issues concerning access or lack thereof to all the relevant steps of their processes (Humphreys 2009). That is, they are opaque in a manner that makes their sanctioning deeply problematic, as we have discussed above. Furthermore, conventional error assessment strategies may simply be inadequate in contexts where software use is prominent (Symons and Horner 2014; 2019; Horner and Symons, 2019). In contrast, simulations that are treated merely as thought experiments, according to Saam, provide "well-founded answers to what-if-thingshad-been-different questions" (Saam 2017, p. 81). That is, while it is very difficult to treat computer simulations as reliable experiments or as theoretically grounded modelling strategies that meet all the epistemic requirements of scientific inquiry, if we treat and/or develop those simulations only as technical artifacts of limited epistemic and imaginative import, then we can deemed them of use as counterfactual testers or instruments to enhance the imagination without having to worry too much about their scientific merit. Simply put, if we agree that they fail to qualify as scientific instruments then we do not need to treat them as such and appropriate attitudes towards their use shall ensue. Moving pictures are in the same category and so are other demonstrative instruments deployed for the illustration of scientific information in non-scientific ways. They are however, not used to reach scientific results. I agree. As opaque technical 
artifacts whose epistemic warrants are not on a par with those required by scientific practice, computer simulations may not in fact enter the catalog of those precision-made scientific instruments whose sanctioning has taken a whole industry ensuring their epistemic standing. However, for now I want to focus on an example that illustrates the inadequacy of views that strongly rely on predictive success as a way to warrant opaque technical artifacts such as computer simulations.

\subsubsection{The Mechanical Oracle}

Consider the following example. Imagine, that once upon a time, there was a civilization in which people had outsourced the task of predicting and controlling their natural and social environments to black boxes with blinking lights. Let's imagine that the story of how these boxes appeared was lost in the mists of history. At first, people might have approached them with trepidation; naturally, they would have been unsure of whether the boxes could be trusted. However, over time, those who trusted the boxes gained an advantage over those who did not. Eventually, the practice developed whereby almost everyone asked the boxes questions with the expectation of receiving predictively accurate answers.

While the mechanical oracles became sources of guidance for their users, the inner workings of these machines remained a mystery. When users asked the boxes for an explanation of their inner workings, the boxes simply refused (or were unable) to provide one. Users assumed that the boxes obeyed the usual physical laws and that their inner workings were in accordance with mathematical principles of computation, but this assumption was simply part of their shared 
commonsense metaphysical background theory rather than having anything directly to do with what was known specifically about the inner workings of the blinking boxes. ${ }^{133}$ Neither the details of the software engineering process that produced the oracles nor the machine learning algorithms that seemed to support their operation were accessible to those who successfully used of the artifacts.

The consensus among users was that the boxes rarely made serious errors with respect to important matters like the paths of hurricanes, the likelihood of epidemics, or the behavior of the economy. They were, by all relevant measures, predictively accurate. For the sake of argument, let us suppose that the users of the oracle are correct in their judgment of its predictive power. And thus, users posed questions to the oracle, took past successes as evidence that future answers were reliable, and granted the boxes significant influence in decision-making. Eventually, social norms developed to the point where it was widely agreed that ignoring the oracle in matters involving important decisions would be unethical. Given the available options and the predictive prowess of these boxes it could also be deemed irrational to not use the boxes in times of crisis. Now the question is, does our use of computer simulation resemble the oracle believer's use of their boxes? More importantly, should our use of computer simulations resemble the practices in our thought experiment?

\footnotetext{
${ }^{133}$ Notice that explicit theoretical or justificatory assumptions from the part of the first people to trust the oracle are not a necessary component of their reliance on the oracle. They could have begun to trust the oracles by default, accident or even superstition (See Skinner's 1947 study “'Superstition' in the Pigeon”. In it, pigeons would continue to perform behaviors they equivocally associated with food rewards solely because the behavior and the distribution coincided successfully in the past).
} 
It is tempting to analogize the situation of the oracle-believers and our own relationship to at least some prominent computational methods. At first glance it seems that in several important contemporary decision-making contexts we already depend heavily on systems that fit the profile of our imagined mechanical oracles. In fact, with the increasing use of machine learning the situation is likely to become opaquer rather than more transparent. It is often the case that we do not have sufficient understanding of the workings of these computational methods to evaluate each of the epistemically relevant steps in its operation. Paul Humphreys described systems of this kind as epistemically opaque (2009). In the case of computational methods, Humphreys in fact suggests that they are essentially epistemically opaque. That is, they are opaque in a manner that may be insurmountable and not just in a manner relative to an agent's position. Likewise, given the complexity of modern software, we cannot fully survey the operation of computational simulations, nor can we be sure that they operate correctly all the time (Symons and Horner 2014: Horner and Symons 2014). It is not surprising then that reliance on software-intensive systems in science has struck some thinkers as questionable and perhaps even unscientific (Newman 2015). If we are ignorant of the method by which the simulation generated its answer, then indeed it seems that we do not have rational basis to believe the answers. As we saw above, non-evidential warrants seem to be immediately inadequate for scientific purposes. However, given our example and the context in which computer simulations are deployed, this intuitive reaction may be too hasty. It is important that we look at it a bit more closely.

In scientific and commercial contexts, it certainly seems as though we have already come to rely heavily on software intensive systems. Given that science and commerce are, for the most part, 
successful, it looks as though our faith in simulation is not unreasonable. However, we are not in the same position as the oracle-believers of the thought experiment. I would argue, for instance that the oracle-believers are not engaged in science in any standard sense of the word. Here I will draw a couple of distinctions to clarify my point. It may be the case that the oracle believers, like many of us in real life, are in fact using a scientific instrument. Something that was put together in a scientific manner through extensive and rigorous precision standards, that was carefully and deliberatively sanctioned with higher epistemic norms in consideration, and that in fact produces scientifically reliable results. And yet, I can still say, that we, as the oracle believers, may use such a scientifically sanctioned artifact in non-scientific ways for non-scientific purposes. In that sense, we are not doing science. Another consideration would be that I, as the oracle believers, could be using a non-scientific artifact in scientific contexts. That is, an instrument that does not meet the norms related to scientific sanctioning deployed to derive results that will be taken as scientific. Yet another point to consider would be an instance in which whether or not the artifact we are using is or is not scientifically sanctioned does not play a part in our considerations for its use in either scientific or non-scientific contexts. In all such cases, we can agree, we are not in fact partaking in scientific inquiry. We are forgoing the norms of scientific practice in that we are not ensuring a sufficiently robust epistemic setting that is associated with scientific practice. Namely, we are not making sure our instrument is a scientific one, that its results can count as scientific, and that we have scientific reasons to deploy it in our setting. In short, we are not doing science. Similarly, while the oracle believers can be said to be successfully deploying their boxes in all kinds of situations, they are not in fact doing science as they do so. Part of the task of the epistemology of computer simulation is to explain the difference between the contemporary scientist's position in relation to epistemically opaque computer simulations and the believers' 
relation to their mechanical oracles in the story above. Another task of the epistemology of computer simulation is to explain how and why computer simulations belong in scientific inquiry. In other words, we still have to figure out whether or not they qualify as scientific instruments and whether or not we should be doing science with them.

Scientists and engineers are often motivated by pragmatic considerations. However, if we restrict ourselves to pragmatic considerations alone we will not be in a position to explain the differences between the oracle-believers and us. As we will see below, pragmatic reasons should be distinguished from properly epistemic reasons.

Given something like the pessimistic meta-induction with respect to the truthfulness of science over the course of history, if our criterion for trust is the history of past successes or failures, then the oracle-believers are certainly more entitled to believe the blinking boxes than we are to believe our latest, best science. The pragmatist must defer to the oracle, since his criterion for deciding between theories is their relative predictive accuracy. While it is certainly true that predictive power is an important desideratum in choosing between theories or models, it is not the only dimension of scientific inquiry (See Symons and Boschetti 2013). Predictions can be accidentally true, predictions can be trivially true, or predictions could be true by magic or clairvoyance. In none of these cases would we ordinarily call the sources of these predictions scientific. ${ }^{134}$

\footnotetext{
${ }^{134}$ A position of this kind leads Quine to concede that he would have to count successful predictions from clairvoyants and telepaths as science (1973, see especially his 1990, pp 20-21). While genuine clairvoyance would
} 
In cases like the oracle, in which the system generates reliably good predictions, most of us would wonder why the oracle works so well. Indeed, it is often the case, in the history of science that natural regularities and correlations become the target of explanation (Nola and Sankey 2014). Thus, for the scientifically-minded, the mysterious predictive power of the oracle would become the explanandum. For the pragmatist, since predictive power is the preeminent criterion of success, explaining predictive power could only be justified in terms of the pursuit of some additional, enhanced, predictive power. ${ }^{135}$ By stipulation, the oracle in our example has maximal predictive power. Therefore, in this case, there is nothing more for a pragmatist to do or to ask for. The inability for the pragmatist to explain the difference between the oracle and ordinary scientific practice is indicative of the weakness of pragmatism as a philosophical position. This also applies to their inability to say why something is or is not a scientific instrument.

One reason in favor of taking a pragmatic stance towards computer simulation is that, given the novelty of their use in scientific inquiry, current simulation practices are more like a craft than a science - in which simulation skills include an interpretative artistry to "reflect and make decisions based on the outcome of computer simulations" (Resch et al. 2017, p. 3). While the craft element of scientific practice is undeniable, its ultimate role in epistemic justification of the

be extremely interesting and useful, on the view that I defend here it would be a phenomenon that is largely orthogonal to science.

${ }^{135}$ In the context of computer simulation, other secondary criteria are also at play for pragmatists. These include simplicity of the algorithm and greater unification of algorithmic calculations. However, these criteria will generally be subordinate to predictive power as a mark of success for the pragmatist. 
results of computer simulation to the consumers of scientific claims will be minimal. Typically, there will be situations in which practitioners in many areas of scientific research will develop skills that are not easily explained in explicit terms. Clearly, even in highly cognitive projects like teaching or scientific research, one develops skills that are difficult to articulate or justify verbally. For example, in some areas of applied mathematics, practitioners use heuristics and trial and error in a way that would be unacceptable in pure mathematics. One might argue for example, that the justification of techniques such as stochastic gradient descent relies on judgment and accumulated expertise. One could generate many examples from experimental science where the use and calibration of instruments involves skills that are not even explicitly teachable, but require something like a period of apprenticeship.

The cultivation of skill and judgment over the course of a career is part of what it means to be an expert and indeed in some areas it makes sense to rely on the judgments of experts simply in virtue of the fact that they are experts. We can grant this and can acknowledge that expertise is often difficult to articulate. ${ }^{136}$ However, as we have seen earlier in this dissertation, the fact that

${ }^{136}$ Thus, for example in cases where an applied mathematician uses techniques that require tacit knowledge or judgment developed through practice to reach some conclusion, we could recognize and rely on this fact in the same way we might recognize that someone who successfully rode a bicycle to work arrived to work without their being able to articulate explicitly, the details of how one rides a bike. The rider's ability to ride a bicycle will not be entirely explainable by the rider, but the fact that he rode to work successfully can be regarded as undeniable and we can believe his claim to have made the journey by bicycle in virtue of knowing that he has the ability to ride a bicycle. 
experts are trustworthy in some contexts is not sufficient for justifying our faith in computer simulations, since computer simulations themselves are not credentialed as experts in the same way as human experts. ${ }^{137}$ We can trust human experts in the use of techniques like stochastic gradient descent in applied mathematics because of their accumulated judgment and experience. However, as we have argued in more detail above, in the case of the computer simulation itself, consumers of scientific claims would be mistaken if they confused the expertise of modelers with the reliability of simulations - the simulation itself does not have the credentials that we would grant an experienced applied mathematician for example.

In response, the defender of expertise in these contexts might argue that expertise is relevant to the consumer of claims generated via simulation insofar as simulations can be certified by experts and experts are reliable sources of judgments about the reliability of computer simulation (Barberousse and Vorms 2014; Beisbart 2017). As we saw above, endorsements from expert practitioners are relevant to the evaluation of simulations, but they are by no means the source of epistemic justification for computer simulations themselves. Another broadly pragmatist defense of computer simulation sees it as a set of engineering tasks tackling one measurement problem at a time (Hubig and Kaminski 2017). While this is an accurate characterization of some simulation practices and their deployment, it does not by itself constitute a substantive reason to adopt a

${ }^{137}$ As we will see, even human expert credentials are not given out the same way we give out credentials to qualify as minimally reasonable interlocutors. I can trust my son, as Burge suggests, when he says that there is no more milk in the fridge based on a minimum intelligibility requirement and an assumption of healthy perceptual capacities. We would apply very different criteria in our choice of scientific experts. 
pragmatist approach to the epistemology of computer simulation. While it is important to account for actual uses and practices of scientific inquiry, simply reporting on the practice does not account for the normative judgment concerning whether we should continue to use or expand the use of that practice. ${ }^{138}$ An epistemology of computer simulations is not merely a sociology of computer simulations. A report on current practices is not an account of whether those practices are the ones that should be in use. Heavily relying on the ways engineers cope with limited epistemic resources to produce some minimal results as evidence of a normative epistemic account of the reliability and trustworthiness of computer simulations risks ignoring this distinction.

However, the problem here is not merely pragmatism's inability to provide satisfactory normative principles for the epistemology of computer simulation. By failing to capture the actual factors by which we rationally come to trust simulations, we risk neglecting the practical processes by which rational trust in science that depends on computational simulation ought to be cultivated in practice. Defenders of Big Data, for example, argued that the way that practitioners were successfully deploying data analysis tools already constituted proper scientific practice and that in many domains, theory and theoreticians were now useless (Steadman 2013;

138 The pragmatic approach seems to presume that science using computational methods is so fluid that the methods and instruments themselves can only be assessed in an ad hoc manner. The focus on reading simulations as sets of isolated measurement tasks misses, for example, the role of carefully curated data and principled theoretical background knowledge in the judgment of the expert interpreter. 
Symons and Alvarado 2016). The dangers of Big Data hubris are now appreciated. ${ }^{139}$ Computer simulation is a broader and more diverse enterprise than Big Data involving a range of distinct techniques and technologies with sensitivity to empirical and theoretical constraints. It therefore has many constraining and correcting influences from the scientists that engage with it (Roush 2015).

The epistemology of computer simulation is a highly complex domain where assumptions concerning the transmission of warrants and the characterization of epistemic entitlements must be understood in relation to the diversity of scientific practices. The actual epistemic situation is fundamentally dissimilar to the situation depicted in my thought experiment. Neither pragmatism, nor an analogy with ordinary epistemic practices will prove useful as guides to the epistemology of computer simulations. Instead, I have argued that trust in simulations should be grounded in empirical evidence, good engineering practice, and established theoretical principles. This is because computer simulations are technical artifacts and as such they ought to be sanctioned accordingly. Technical artifacts that make it into the annals of scientific instruments dot merely do so in virtue of entitlements or because they are already partaking in undergoing practice. Rather, they are included or discarded by arduous and meticulous procedures of that confront them to high epistemic standards to assess whether or not they are fit

${ }^{139}$ An example of the pitfalls of atheoretical Big Data studies, is discussed by Lazer et al. (2014). Ultimately, many advocates of Big Data were misguided in spite of being able to point to well-established practices, successful predictive patterns and expertise within the data science community. 
for the task. Without these constraints, computer simulation risks becoming little more than unmoored speculation. 


\section{Conclusion}

In this dissertation I have provided an overview of the most relevant philosophical discussions in the philosophy of computer simulations of the last two decades as well as of the most recent philosophical positions concerning their nature, significance and role in scientific inquiry. I showed how the field transitioned from its early, narrow, understanding of computer simulations as mere extensions of the formal content they were once made to simulate to acknowledging their extra-formal elements as epistemically essential for their understanding (Winsberg, 2010). This transition pushed the concept of computer simulation to be understood as something closer to empirical practices such as experiments. This lasted almost a decade, in which philosophers, engineers and sociologists who accepted computer simulations as experiments wondered exactly what kind of experiments they could possibly be (Beisbart, 2018). As indicative of this dilemma, Nicole Saam (2017) reviews the past two decades of this debate in the philosophy of simulation and identifies four different positions: simulation as a special type of thought experiment, simulation as a special type of experiment, simulations as fundamentally different from thought experiments, and simulations as fundamentally different from laboratory experiments. The philosophy of simulation, in fact, has devoted many years and much ink has been spilled exploring these differentiations (Lenhard, 2007; Barberousse et al., 2008; 2009; Parker, 2009; Durán, 2018). 
When faced with deep philosophical challenges that questioned the similarity of computer simulations to experiments, some in this camp, who nevertheless acknowledged the extra-formal elements of computer simulations, reverted to deflationary views of what was meant by experiment. This way, they included those practices that consisted in the manipulation of purely formal elements such as theoretical values and measurements (Morrison, 2015; Schiaffonati, 2016). Others pushed the concept of computer simulation further towards the outside of both the formal content they inherently manipulate and the experimental settings in which they are used to include the sociological aspects surrounding their design, development and deployment (Beisbart and Saam, 2018). Computer simulations, this view suggested, are more like a practice. This broadening move contributed to the development of important questions regarding the diverse skills, communities and methods necessary to their implementation. Together, all these views have substantially contributed to the overall understanding of computer simulations.

However, In this dissertation I argued that these views also served as evidence of something else: the focus on the essential formal features of computer simulations coupled with the acknowledgment of their experiment-like and engineered aspects served to indicate that they are in fact best understood as something else than any of their aforementioned constituents. In this dissertation I argued that computer simulations are distinct artifacts with distinct aims and tasks from those of the content they manipulate, the experimental settings in which they are deployed and from the sociological aspects that surround their construction. They are the things with which some of the above elements can be carried out. In particular, I argued that computer simulations are the kinds of things that are best understood as technical artifacts of the kind designed, developed and deployed in scientific inquiry. In virtue of this, I concluded that 
computer simulations are best understood as instruments rather than as models, experiments or practices.

To claim that computer simulations are instruments, that they ought to be treated as instruments or that they should be understood as instruments, however, does not entail that they are just like other instruments. They are not. Computer simulations are in fact hybrid instruments, composed of a multiplicity of components and capable of many tasks. Because their distinct function is to simulate, they can take over the tasks of many different processes and artifacts in the laboratory: they can simulate an entire experimental setup, an analytic method, the interventions done by another instrument, or even the dynamic features of natural phenomena. This is something I argued by showing the hybridity of computer simulations vis-a-vis existing taxonomies of scientific instruments.

Throughout this dissertation, I also suggested that computer simulations may indeed be novel additions to inquiry, as many have suggested, but they are only so as instruments and not as a separate branch of inquiry onto themselves. This is the kind of novelty related to computer simulations that ought to be investigated in the future. That is, rather than investigating the novelty of computer simulations as a whole scientific method onto itself, rather than investigating their novelty as a possible third branch of science, we should investigate what kind of novel instrument they are. In so arguing I do not only hope to have established computer simulations as a novel and interesting addition to scientific inquiry but I hope to also have brought forth once more the old idea that science already has a third branch of inquiry that has been neglected by philosophers: namely instruments. 
After establishing my view of computer simulations as instruments, I also showed that the heterogeneity of computer simulation projects, emphasized by what I called the 'practice view' of computer simulations, was not sufficient reason to consider their understanding in terms of instruments as misguided. Rather, I argued, if this was the case, even those devices that had enjoyed a status as scientific instruments for centuries would have to be excluded from the concept. This is because instruments such as the telescope were also the product of heterogeneous communities, components and practices, just like computer simulations. If the argument from heterogeneity was to have any effect on considering computer simulations as instruments, telescopes would suffer too. This showed the argumentative strategy regarding heterogeneity to be too far reaching and hence inadequate to exclude computer simulations from being understood as instruments.

The characterization of computer simulations as hybrid instruments should serve as a starting point of a more accurate understanding of their nature, significance and role within scientific inquiry. As it is, this view already shows important ramifications for the way we can and cannot accept their ubiquitous presence in scientific laboratories. In particular, this view offers a framework that allows us to question whether and when we are using them as an oracle believer would use their oracle or when we are using them as we use and sanction other scientific instruments; whether we trust them because they have withstood a healthy and rigorous dose of empirical skepticism or simply in virtue of some pragmatic version of technological determinism. As I argued in the final section of this dissertation, whenever the latter is the case, we can easily identify it as an instance that fails to meet important norms in scientific inquiry, particularly those that ensure it as a slightly better way of overcoming our very fallible epistemic 
limitations. Warrants such as epistemic entitlements, which require no evidential effort on the part of the epistemic agent, are not adequate to sanction recently introduced instruments in scientific inquiry. To suggest so is to miss the point of science, which is, at the very least, to overcome some of our deeply rooted and ordinary epistemic limitations.

Computer simulations have the capacity to revolutionize the way we do science, but revolutions can have catastrophic results. By treating them as instruments we can more carefully discern the warrants that justify their use and we can more carefully ensure that the changes they bring about are consistent with the superior epistemic requirements at the center of scientific inquiry. 


\section{References}

Adler, J. (2015). Epistemological problems of testimony. In E. N. Zalta (Ed.), The stanford encyclopedia of philosophy (summer 2015 edition).

https://plato.stanford.edu/archives/sum2015/entries/testimony-episprob/. Accessed 20 Dec 2018.

Alvarado, R., \& Humphreys, P. (2017). Big data, thick mediation, and representational opacity. New Literary History, 48(4), 729-749.

Arkoudas, K., \& Bringsjord, S. (2007). Computers, justification, and mathematical knowledge. Minds and Machines, 17(2), 185-202.

Audi, R. (1997). The place of testimony in the fabric of knowledge and justification. American Philosophical Quarterly, 34(4), 405-422.

Baird, Davis. Thing knowledge: A philosophy of scientific instruments. Univ of California Press, 2004.

Barberousse, A., Franceschelli, S., \& Imbert, C. (2009). Computer simulations as experiments. Synthese, 169(3), 557-574.

Barberousse, A., \& Vorms, M. (2014). About the warrants of computer-based empirical knowledge. Synthese, 191(15), 3595-3620.

Barberousse, Anouk, and Julie Jebeile. "How Do the Validations of Simulations and Experiments Compare?." Computer Simulation Validation. Springer, Cham, 2019. 925-942.

Beebee, H. (2001). Transfer of warrant, begging the question and semantic externalism. The Philosophical Quarterly, 51(204), 356-374.

Belfer, Israel. "The Info-Computation Turn in Physics." Turing-100. 2012.

Beisbart, C. (2017). Advancing knowledge through computer simulations? A socratic exercise. In M. Resch, A. Kaminski, \& P. Gehring (Eds.), The science and art of simulation I (pp. 153174). Berlin: Springer.

Beisbart, Claus. "Are computer simulations experiments? And if not, how are they related to each other?." European Journal for Philosophy of Science 8.2 (2018): 171-204.

Beisbart, Claus, and Nicole J. Saam, eds. Computer Simulation Validation: Fundamental Concepts, Methodological Frameworks, and Philosophical Perspectives. Springer, 2018.

Borge, S. (2003). The word of others. Journal of Applied Logic, 1(1-2), 107-118. 
Boschetti, F., \& Symons, J. (2011). Why models' outputs should be interpreted as predictions. In Proceedings of the International Congress on Modelling and Simulation (MODSIM 2011), Perth, Australia (pp. 12-16).

Boschetti, F., Fulton, E., Bradbury, R., \& Symons, J. (2012). What is a model, why people don't trust them and why they should. In M. R. Raupach (Ed.), Negotiating our future: Living scenarios for Australia to 2050 (pp. 107-118). Australian Academy of Science.

Burge, T. (1993). Content preservation. The Philosophical Review, 102(4), 457-488.

Burge, T. (1998). Computer proof, apriori knowledge, and other minds: The sixth philosophical perspectives lecture. Nô̂s, 32(S12), 1-37.

Dalmedico, Amy Dahan. "History and epistemology of models: Meteorology (1946-1963) as a case study." Archive for history of exact sciences 55, no. 5 (2001): 395-422.

Davidson, D. (1973). Radical interpretation. Dialectica, 27(3-4), 313-328.

Davies, M. (2004) II-Martin Davies: Epistemic entitlement, warrant transmission and easy knowledge. In Aristotelian Society supplementary volume (Vol. 78(1)). Oxford: The Oxford University Press.

Dretske, F. (2000). Entitlement: Epistemic rights without epistemic duties? Philosophy and Phenomenological Research, 60(3), 591-606.

Dubucs, Jacques. "Simulations et modélisations." Pour la science 300 (2002): 156-158.

Durán, Juan M. "Varieties of Simulations: From the Analogue to the Digital." In The Science and Art of Simulation I, pp. 175-192. Springer, Cham, 2017.

Durán, Juan Manuel. Computer Simulations in Science and Engineering. Springer, 2018.

Durán, Juan M., and Nico Formanek. "Grounds for trust: Essential epistemic opacity and computational reliabilism." Minds and Machines 28.4 (2018): 645-666.

Frans, Joachim, and Laszlo Kosolosky. "Mathematical Proofs in Practice: Revisiting the reliability of published mathematical proofs." THEORIA. Revista de Teoría, Historia y Fundamentos de la Ciencia 29.3 (2014): 345-360.

Fresco, N., \& Primiero, G. (2013). Miscomputation. Philosophy \& Technology, 26(3), 253-272.

Frigg, R., \& Reiss, J. (2009). The philosophy of simulation: Hot new issues or same old stew? Synthese, 169(3), 593-613.

Galison, Peter. "Computer simulations and the trading zone." (1996). 
Gransche, Bruno. "The Art of Staging Simulations: Mise-en-scène, Social Impact, and Simulation Literacy." The Science and Art of Simulation I. Springer, Cham, 2017. 33-50.

Gramelsberger, G. (2011). Generation of evidence in simulation runs: Interlinking with models for predicting weather and climate change. Simulation \& Gaming, 42(2), 212-224.

Gehring, Petra. "Doing Research on Simulation Sciences? Questioning Methodologies and Disciplinarities." The Science and Art of Simulation I. Springer, Cham, 2017. 9-21.

Grüne-Yanoff, Till. "Seven problems with massive simulation models for policy decisionmaking." The Science and Art of Simulation I. Springer, Cham, 2017. 85-101.

Hacking, Ian, and Jan Hacking. Representing and intervening: Introductory topics in the philosophy of natural science. Cambridge university press, 1983.

Hartmann, Stephan. "The world as a process." In Modelling and simulation in the social sciences from the philosophy of science point of view, pp. 77-100. Springer, Dordrecht, 1996.

Harré, R. "The Materiality of Instruments in a Metaphysics for Experiments. The Philosophy of Scientific Experimentation. H. Radder. Pittsburgh." (2003): 19-38.

Heidelberger, Michael. "Theory-ladenness and scientific instruments in experimentation." (2003): 8-151.

Heilbron, John L. "Some uses for catalogues of old scientific instruments." Essays on Historical Scientific Instruments..., Aldershot, Variorum (1993): 1-16.

Heppner, Frank, and Ulf Grenander. "A stochastic nonlinear model for coordinated bird flocks." The ubiquity of chaos 233 (1990): 238.

Holzmann, G. J. (2015). Code inflation. IEEE Software, 2, 10-13.

Horner, J., \& Symons, J. (2014). Reply to Angius and Primiero on software intensive science. Philosophy \& Technology, 27(3), 491-494.

Horner, J. K., \& Symons, J. (2019). Understanding Error Rates in Software Engineering: Conceptual, Empirical, and Experimental Approaches. Philosophy \& Technology, 1-16.

Hu R., \& Ru, X. (2003) Differential equation and cellular automata models. In IEEE Proceedings of the International Conference on Robotics, Intelligent Systems and Signal Processing (pp. 1047-1051).

Hubig, C, \& Kaminski, A. (2017). Outlines of a pragmatic theory of truth and error in computer simulation. In M. Resch, A. Kaminski, \& P. Gehring (Eds.), The science and art of simulation I (pp. 121-136). Cham: Springer. 
Humphreys, P. (2004). Extending ourselves: Computational science, empiricism, and scientific method. Oxford: Oxford University Press.

Humphreys, P. (2009). The philosophical novelty of computer simulation methods. Synthese, $169(3), 615-626$.

Humphreys, P. (2009). Network epistemology. Episteme, 6(2), 221-229.

IPCC (2001). Climate Change 2001: Synthesis Report. Contribution of Working Group I, II, and III to the Third Assessment Report of the Intergovernmental Panel on Climate Change, Cambridge, England: Cambridge University Press

Jenkins, C. S. (2007). Entitlement and rationality. Synthese, 157(1), 25-45.

Kaminski, Andreas. "Der Erfolg der Modellierung und das Ende der Modelle. Epistemische Opazität in der Computersimulation." (2017).

Kaminski, Andreas, Michael M. Resch, and Uwe Küster. Mathematische Opazität. Über Rechtfertigung und Reproduzierbarkeit in der Computersimulation: Jahrbuch Technikphilosophie (2018)

Kaufmann and L. L. Smarr. Supercomputing and the Transformation of Science. Scientific American Library, 1993

Keller, EvelynFox. Models, simulation, and “computer experiments”. na, 2003.

King, Henry C. The history of the telescope. Courier Corporation, 2003.

Koyré, Alexandre. From the closed world to the infinite universe. Vol. 1. Library of Alexandria, 1957.

Kroes, P. A., and A. W. M. Meijers. "The dual nature of technical artefacts." Studies in History and Philosophy of Science 37.1 (2006): 1-4.

Kroes, Peter. "Design methodology and the nature of technical artefacts." Design studies 23, no. 3 (2002): 287-302.

Kroes, Peter. "Coherence of structural and functional descriptions of technical artefacts." Studies in History and Philosophy of Science Part A 37, no. 1 (2006): 137-151.

Kroes, Peter. "Engineering and the dual nature of technical artefacts." Cambridge journal of economics 34.1 (2009): 51-62.

Kroes, Peter. Technical artefacts: Creations of mind and matter: A philosophy of engineering design. Vol. 6. Springer Science \& Business Media, 2012. 
Kuhl, F., Dahmann, J., \& Weatherly, R. (2000). Creating computer simulation systems: An introduction to the high-level architecture. Upper Saddle River: Prentice Hall.

Kuhn, Thomas S. "The structure of scientific revolutions." Chicago and London (1962

Lackey, J. (1999). Testimonial knowledge and transmission. The Philosophical Quarterly, 49(197), 471-490.

Lazer, D., Kennedy, R., King, G., et al. (2014). The parable of Google Flu: Traps in big data analysis. Science, 434, 343.

Lenhard, Johannes. "Computer simulation: The cooperation between experimenting and modeling." Philosophy of Science 74.2 (2007): 176-194.

Maley, Corey J. "Analog and digital, continuous and discrete." Philosophical Studies 155.1 (2011): 117-131.

Marcovich, Anne, and Terry Shinn. "How scientific research instruments change: A century of Nobel Prize physics instrumentation." Social Science Information 56.3 (201

McEvoy, M. (2008). The epistemological status of computer-assisted proofs. Philosophia Mathematica, 16(3), 374-387.

McEvoy, M. (2013). Experimental mathematics, computers and the a priori. Synthese, 190(3), 397-412.

McGlynn, A. (2014). On Epistemic Alchemy. In D. Dodd, \& E. Zardini (Eds.), Scepticism and Perceptual Justification. (pp. 173-189), OUP Oxford.

Metropolis, Nicholas. "The beginning of the Monte Carlo method." Los Alamos Science 15.584 (1987): 125-130.

Moretti, L., \& Piazza, T. (2013). When warrant transmits and when it doesn't: Towards a general framework. Synthese, 190(13), 2481-2503.

Morgan, Mary S., Margaret Morrison, and Quentin Skinner, eds. Models as mediators:

Perspectives on natural and social science. Vol. 52. Cambridge University Press, 1999.

Morgan, M. S. (2005). Experiments versus models: New phenomena, inference and surprise. Journal of Economic Methodology, 12(2), 317-329.

Morrison, Margaret. "Models, measurement and computer simulation: the changing face of experimentation." Philosophical Studies 143, no. 1 (2009): 33-57.

Morrison, M. (2015). Reconstructing reality. Oxford: Oxford University Press. 
Nariya, Maulik K., et al. "Mathematical model for length control by the timing of substrate switching in the type III secretion system." PLoS computational biology 12.4 (2016): e1004851.

Naylor, T. H., Balintfy, J. L., Burdick, D. S., \& Chu, K. (1966). Computer simulation techniques. New York: Wiley.

Newman, J. (2015). Epistemic opacity, confirmation holism and technical debt: Computer simulation in the light of empirical software engineering. In International conference on history and philosophy of computing (pp. 256-272). Springer.

Nola, R., \& Sankey, H. (2014). Theories of scientific method: An introduction. Abingdon: Routledge.

Norton, S., \& Suppe, F. (2001). Why atmospheric modeling is good science (pp. 67-105). Changing the atmosphere: Expert knowledge and environmental governance.

Oreskes, N., Shrader-Frechette, K., \& Belitz, K. (1994). Verification, validation, and confirmation of numerical models in the earth sciences. Science, 263(5147), 641-646.

Oreskes, N. (2004). The scientific consensus on climate change. Science, 306(5702), 1686-1686.

Parnas, David L., A. John Van Schouwen, and Shu Po Kwan. "Evaluation of safety-critical software." Communications of the ACM 33, no. 6 (1990): 636-648.

Parker, W. S. (2009). Does matter really matter? Computer simulations, experiments, and materiality. Synthese, 169(3), 483-496.

Petersen, A. C. (2012). Simulating nature: a philosophical study of computer-simulation uncertainties and their role in climate science and policy advice. Chapman and Hall/CRC.

Phillips, Norman A. "The general circulation of the atmosphere: A numerical experiment." Quarterly Journal of the Royal Meteorological Society 82.352 (1956): 123-164.

Pias, Claus. "On the epistemology of computer simulation." Zeitschrift für Medien-und Kulturforschung 2011.1 (2011): 29-54.

Pincock, C. (2011). Mathematics and scientific representation. Oxford: Oxford University Press.

Primiero, Giuseppe. "On the ontology of the computing process and the epistemology of the computed." Philosophy \& Technology 27, no. 3 (2014): 485-489.

Primiero, Giuseppe. "A Minimalist Epistemology for Agent-Based Simulations in the Artificial Sciences." Minds and Machines 29.1 (2019): 127-148.

Pryor, J. (2012). When warrant transmits. In W. Crispin (Ed.), Mind, meaning, and knowledge: Themes from the philosophy of Crispin Wright (pp. 269-303). Oxford: University Press. 
Quine, W. V. (1973). The roots of reference. La Salle, Ill: Open Court.

Quine, W. (1960). Word and object. MIT press.

Resch, M. M. (2013) What's the result? Thoughts of a Center Director on Simulation. In Computer Simulation and the Changing Face of Scientific Experimentation, ed. Juan M. Durán and Eckhardt Arnold, 233-246. Newcastle: Cambridge scholars Publishing.

Resch, M. M., Kaminski, A., \& Gehring, P. (Eds.). (2017). The science and art of simulation I: Exploring-understanding-knowing. Berlin: Springer.

Resnik, M. (1997). Mathematics as a science of patterns. New York: Oxford University Press. Roush, S. (2015). The epistemic superiority of experiment to simulation. Synthese, 169, 1-24.

Reynolds, Craig W. Flocks, herds and schools: A distributed behavioral model. Vol. 21. No. 4. ACM, 1987.

Ruphy, S. (2011). Limits to modeling: Balancing ambition and outcome in astrophysics and cosmology. Simulation \& Gaming, 42(2), 177-194.

Ruphy, S. (2015). Computer simulations: A new mode of scientific inquiry? In S. O. Hansen (Ed.), The role of technology in science: Philosophical perspectives (pp. 131-148). Dordrecht: Springer.

Saam, N. J. (2017). Understanding social science simulations: Distinguishing two categories of simulations. In M. Resch, A. Kaminski, \& P. Gehring (Eds.), The science and art of simulation I (pp. 67-84). Cham: Springer.

Saam, Nicole J. "What is a computer simulation? A review of a passionate debate." Journal for General Philosophy of Science 48.2 (2017): 293-309.

Saam, Nicole J., and Andreas Harrer. "Simulating norms, social inequality, and functional change in artificial societies." Journal of Artificial Societies and Social Simulation 2.1 (1999): 2.

Schelling, Thomas C. "Dynamic models of segregation." Journal of mathematical sociology 1.2 (1971): 143-186.

Schiaffonati, Viola. "Stretching the traditional notion of experiment in computing: Explorative experiments." Science and engineering ethics 22.3 (2016): 647-665.

Schweber, Silvan, \& Watcher, M. (2000). Complex Systems, Modelling and Simulation. Studies in History and Philosophy of Science Part B: Studies In History and Philosophy of Modern Physics, 31(4), 583-609.

Simon, Herbert A. "The sciences of the artificial." Cambridge, MA (1969). 
Simondon, Gilbert. "On the mode of existence of technical objects, trans." Ninian Mel lamphy, unpublished. University of Western Ontario: London, Ontario (1958).

Simoulin, Vincent. "Une communauté instrumentale divisée... et réunie par son instrument." Revue d'anthropologie des connaissances 1.2 (2007): 221-241.

Simoulin, Vincent. "An instrument can hide many others: Or how multiple instruments grow into a polymorphic instrumentation." Social Science Information 56.3 (2017): 416-433.

Steadman, I. (2013). Big data and the death of the theorist. Wired Online, 25, 2013.

Sugden, Robert. "Credible worlds: the status of theoretical models in economics." Journal of economic methodology 7.1 (2000): 1-31.

Symons, J. (2008). Computational models of emergent properties. Minds and Machines, 18(4), 475-491.

Symons, J., \& Alvarado, R. (2016). Can we trust big data? Applying philosophy of science to software. Big Data \& Society, 3(2), 2053951716664747.

Symons, John, and Ramón Alvarado. "Epistemic entitlements and the practice of computer simulation." Minds and Machines 29.1 (2019): 37-60.

Symons, J., \& Boschetti, F. (2013). How computational models predict the behavior of complex systems. Foundations of Science, 18(4), 809-821.

Symons, J., \& Horner, J. (2014). Software intensive science. Philosophy \& Technology, 27(3), 461-477.

Symons, J., \& Horner, J. (2017). Software error as a limit to inquiry for finite agents: challenges for the post-human scientist. In T. Powers (Ed.), Philosophy and computing: Essays in epistemology, philosophy of mind, logic, and ethics. Philosophical Studies Series (Vol. 128, pp. 85-97). Berlin: Springer. Berlin: Springer.

Symons, J., \& Horner, J. (2019). Why There is no General Solution to the Problem of Software Verification. Foundations of Science, 1-17.

Szabó, Barna, and Ricardo Actis. "Simulation governance: Technical requirements for mechanical design." Computer Methods in Applied Mechanics and Engineering 249 (2012): 158168.

Turner, R. (2018). Computational artifacts: towards a philosophy of computer science. Springer.

Tymoczko, T. (1979). The four-color problem and its philosophical significance. Journal of Philosophy, 76, 57-82. 
Vallor, S. (2017). AI and the automation of wisdom. In T. Powers (Ed.), Philosophy and computing: Essays in epistemology, philosophy of mind, logic, and ethics. Philosophical Studies Series (Vol. 128, pp. 161-178). Berlin: Springer.

Van Helden, Albert, and Thomas L. Hankins. "Introduction: Instruments in the history of science." (1994): 1-6.

Van Helden, Albert. Galileo and the telescope. Connexions, Rice University, 2004.

van de Velde, Louis R. "Computers for field artillery." Papers presented at the May 3-5, 1960, western joint IRE-AIEE-ACM computer conference. ACM, 1960.

Von Neumann, John, and Goldstine Brucks. "Preliminary discussion of the logical design of an electronic computing instrument." (1946).

Von Neumann, John, and Arthur W. Burks. "Theory of self-reproducing automata." IEEE Transactions on Neural Networks 5.1 (1966): 3-14.

Warner, Deborah Jean. "What is a scientific instrument, when did it become one, and why?." The British journal for the history of science 23.1 (1990): 83-93.

Weisberg, Michael. Simulation and similarity: Using models to understand the world. Oxford University Press, 2012.

Williams, M. (2000). Dretske on epistemic entitlement. Philosophy and Phenomenological Research, 60(3), 607-612.

Winsberg, E. (2010). Science in the age of computer simulation. Chicago: University of Chicago Press.

Winsberg, Eric, and Ali Mirza. "Considerations from the philosophy of simulation." The Routledge Handbook of Scientific Realism (2017): 250.

Winsberg, E. (2019). Computer simulations in science. In E. N. Zalta (Ed.), The Stanford encyclopedia of philosophy (summer 2015 edition).

http://plato.stanford.edu/archives/sum2015/entries/simulations-science/. Accessed 20 Dec 2018.

Wright, C., \& Davies M. (2004) On epistemic entitlement. In Proceedings of the Aristotelian society, supplementary volumes (Vol. 78, pp. 167-245). www.jstor.org/stable/4106950.

Accessed 20 Dec 2018.

Zik, Y. 1999. "Galileo and the Telescope: Th e Status of Theoretical and Practical Knowledge and Techniques of Measurement and Experimentation in the Development of the Instrument.”Nuncius. Anno XI V. fas c1: 31-69. 
Zik, Yaakov. "Science and Instruments: The telescope as a scientific instrument at the beginning of the seventeenth century." Perspectives on Science 9.3 (2001): 259-284. 